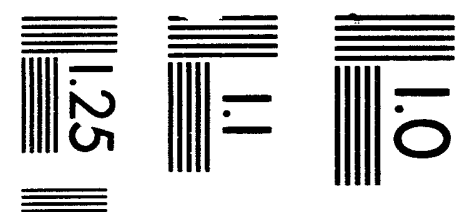

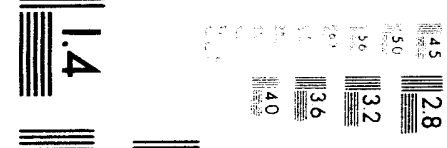

$$
\begin{aligned}
& \text { FE FE }
\end{aligned}
$$



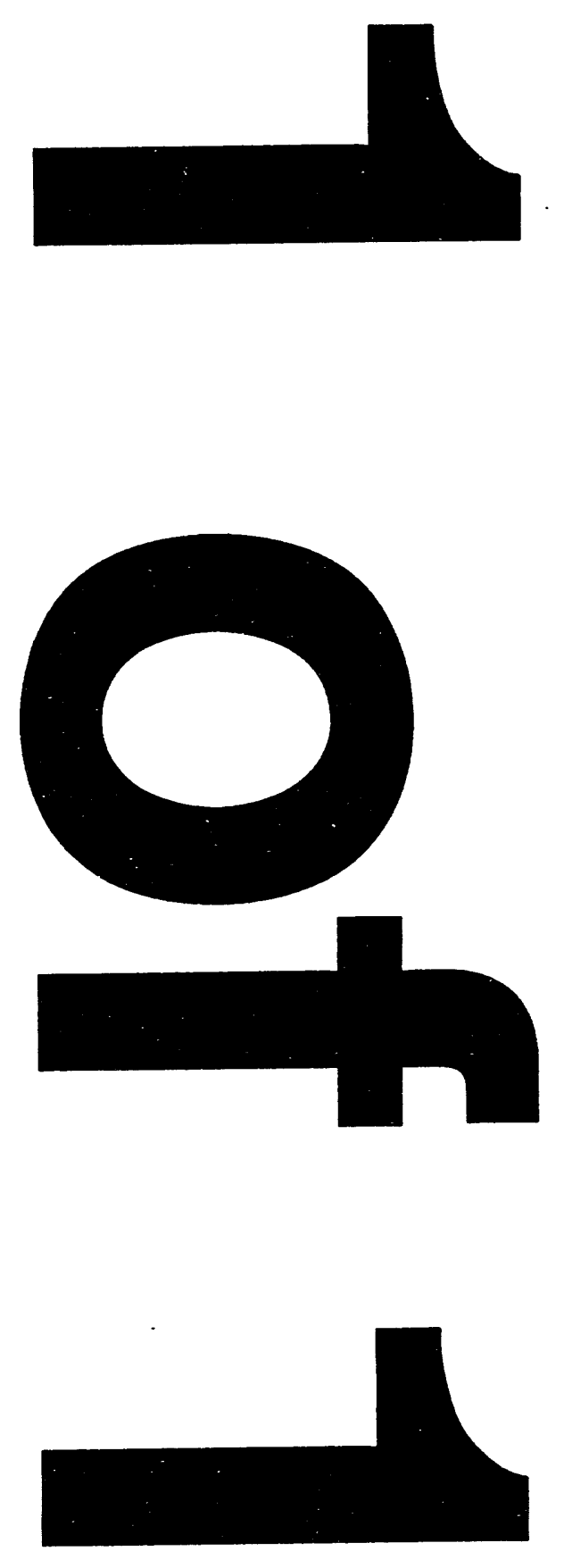


\title{
TECHNOLOGY DEVELOPMENT FOR IRON FISCHER-TROPSCH CATALYSTS
}

\author{
CONTRACT DE-AC22-90PC90055
}

\author{
Technical Progress Report No. 8 \\ 7/1/92 - 9/30/92
}

DISCLAIMER

\begin{abstract}
This report was prepared as an account of work sponsored by an agency of the United States This report was prepare aner their Government. Neither the Cnited States Governmen, or assumes any legal liability or responsiemploce, maked, or bility for the accuracy, completeness, or usefulness of any information, apparatus, product, or process disclosed, or represents that its use would not infringe privately owed trade name, trademark, ence herein to any specific commercial product, process, or serimply its endorsement, recommanufacturer, or otherwise does not necessarily constitute or imply its en thereof. The views mendation, or favoring by the United States Government or any agency thereof. The vect the and opinions of authors expressed herein do not

United States Government or any agency thereof.
\end{abstract}

\section{prepared for}

\section{Pittsburgh Energy Technology Center \\ Department of Energy Pittslurgh, Pennsylvania}

$$
\text { \% }
$$

prepared by

\section{UOP}

25 E. Algonquin Road

Des Plaines, nlinois

PATEIT CIEARED BY CHICAGO
OPC ON Nivember 15,1993 
TECHNOLOGY DEVELOPMENT FOR IRUN FISCHER-TROPSCH CATALYSTS

Contract DE-AC22-90PC90055

Technical Progress Report No. 8

(7/1/92-9/30/92)

by

Robert R. Frame and Hemant B. Gala

UOP

25 E. Algonquin Road

Des Plaines, Illinois

\section{Contract Objective}

The objectives of this contract are to develop a technology for the production of active and stable iron $(\mathrm{Fe})$ Fischer-Tropsch catalysts for use in slurry-phase synthesis reactors and to develop a scaleup procedure for large-scale synthesis of such catalysts for process development and longterm testing in slurry bubble-column reactors. With a feed containing hydrogen $\left(\mathrm{H}_{2}\right)$ and carbon monoxide ( $\mathrm{CO}$ ) in the molar ratio of 0.5 to 1.0 to the slurry bubble-column reactor, the catalyst performance target is $88 \% \mathrm{CO}+\mathrm{H}_{2}$ conversion at a minimum space velocity of $2.4 \mathrm{NL} / \mathrm{hr} / \mathrm{gFe}$. The desired sum of methane and ethane selectivities is no more than $4 \%$, and the conversion loss per week is not to exceed $1 \%$.

\section{Contract Tasks}

Task 1.0: Catalyst development

1.1: Technology assessment

1.2: Precipitated catalyst preparation method development

1.3: Novel catalyst preparation methods investigation

1.4: Catalyst pretreatment

1.5: Catalyst characterization

Task 2.0: Catalyst testing

Task 3.0: Catalyst aging studies

Task 4.0: Preliminary design and cost estimate of a catalyst synthesis facility 


\section{Scope of Work Reported}

The work reported in this technical progress report covers a new technique developed to add potassium $(\mathrm{K})$ to the iron-copper oxide catalyst and testing of two catalysts prepared by using this technique.

The use of an alkali promoter like $\mathrm{K}$ to suppress the formation of methane is widely practiced. The objective of developing a novel $\mathbf{K}$-addition technique was to simplify the method of catalyst preparation by reducing the steps involved and to have an external control over the selectivity of the iron catalyst to methane formation.

In the new method a potassium salt of a long-chain carboxylic acid (e.g. potassium laurate) is added to a mixture of the iron-copper oxide composition and an oil (typically a $\mathrm{C}_{30}$ oil) in the slurry autoclave. The resulting mixture can be activated by a standard method to produce a slurry-phase Fischer-Tropsch catalyst. In performance such a catalyst exhibits comparable $\mathrm{H}_{2}$ and $\mathrm{CO}$ conversions and product selectivities as a catalyst prepared by the aqueous impregnation of potassium carbonate followed by calcination.

\section{Experimental Procedures}

For tests reported in this technical progress report, UOP's standard procedure for activation and testing was used. The slurry autoclave reactor used for these runs is contained in a hot box which is maintained at $130^{\circ} \mathrm{C}$. The liquid and gaseous products are separately withdrawn but taken to the same traps. There are three traps in series the first is in the hot box, the second and third are outside of the not box and maintained at ice and dry ice/acetone temperatures, respectively. A small slip-stream of the product before the ice trap is taken to a gas chromatograph (GC), GC-1, for an on-line boiling point analysis. A second slip-stream (after the ice trap) is taken to a second GC, GC-2, for an analysis of the light gases $\left(C_{1}\right.$ to $\left.C_{4}\right)$. Periodically the total product $\left(\mathrm{CO}_{2}\right.$-free basis) is analyzed by a mixture of off- and on-line methods as follows: the gases by on-line GC, the contents of the hot box trap (wax) by off-line gel phase chromatography (GPC), and the contents of two cold traps by off-line GC.

\section{Run Summary}

\section{Run 42}

Ten grams of potassium laurate were used with seventy-three grams of iron-copper oxide (Table 1). The iron : potassium weight ratio was $100: 3.2$. Based on previous work with catalysts prepared by aqueous impregnation of potassium carbonate onto iron-copper oxide followed by calcination, this ratio is higher than needed for optimum conversion and low methane + ethane selectivity (Report No. 4, current contract). However, at the outset of this work it was not known whether there would be a correspondence between the two potassium addition methods, therefore, two levels of potassium were investigated: the level used in this run and the lower level used in Run 43. 
The conditions for initial part of this nin were the same as those used in previous runs with catalysts prepared by the potassium imp egnation method. After about fifty hours-on-stream the conversions appeared to be lined-out, for instance, the CO conversion was $60 \%$. This conversion is about what one would expect based on previous work in this laboratory for similar $\mathrm{K}$-level catalysts resulting from potassium carbonate impregnation.

Plots of conversions and light product selectivities (GC-2 data) vs. hours-on-stream are in the Appendix as Figures A-1 to A-6. The methane selectivity was quite low as expected due both to the relatively low conversion and the high level of potassium. Previous work in these and other laboratories has shown that methane selectivity decreases as the potassium level increases or the conversion decreases. Selectivities to the other light products were also low, this again was expected based on previous work. A plot of olefin/paraffin ratio vs. carbon number is in Figure A-7. These data are from GC-1 and illustrate the known fact that the Fischer-Tropsch product becomes less olefinic as the carbon number increases.

The liquid level in the autoclave is always allowed to build to a target from the initial low level, but thereafter the level is maintained by periodic liquid withdrawals. A constant level at about seven inches from the bottom of the autoclave is desired. The liquid level can be determined by measurements of the internal reactor temperatures vs. distance from bottom of the reactor. Figure A-8 is a plot of the liquid level vs time and shows that the liquid level was maintained at seven inches except for a brief period around sixty-five hours-on-stream.

At 145 hours-on-stream the space velocity was halved, as a result the conversions increased to levels very nearly the same as in the earlier Run 37 which utilized the most active of this contract's potassium carbonate impregrated catalysts, but at the initial, higher space velocity. Conversions and selectivities from Runs 37 and 42 are summarized in Table 1. In Run 42 the increased conversion induced by the lower space velocity also resulted in an increase in the methane selectivity as expected. However, the methane selectivity at $70 \% \mathrm{CO}$ conversion was significantly lower than that of the Run 37 catalyst at a similar (76\%) CO conversion. The other light hydrocarbon selectivities did not seem to be affected by the space velocity change. However, there were increases in the light alcohol selectivities after the space velocity change.

A complete analysis of the total product was made for three twenty-four hr periods of this run. These three periods covered the following times-on-stream: $25-48 \mathrm{hrs}, 97-120 \mathrm{hrs}$ and 145-168 hrs. The product composition was determined by on-line $\mathrm{GC}$ analysis of the product gas $\left(\mathrm{C}_{1}\right.$ $\mathrm{C}_{4}$ ), off-line GC analyses of the contents of the wet and dry ice traps and off-line GPC analysis of the contents of the hot box trap. The traps do not do an adequate job of collecting the $C_{S}$ 's and $\mathrm{C}_{6}$ 's, therefore, these concentrations were no doubt higher than found. A sample wax GPC analysis is shown in Figure A-9. Wax and GC analyses are summarized in three Lotus spreadsheets, one each for the three periods, Figures A-10 to A-12. From these analyses the weight percent of the total product at each carbon number on a $\mathrm{CO}_{2}$-free basis (sum from all traps) as well as the natural logarithm of the ratio of wt \% at a carbon no. to the carbon no. was calculated. These data are summarized in a wt $\%$ of total product vs carbon number plot (Figure A-13), and Schulz-Flory plots and attached as Figures A-14 to A-19. Figure A-13 illustrates 
that the values for $C_{5}$ and $C_{6}$ are low, this is because the traps do not do an adequate job of trapping these products. The Schulz-Flory plots include full range and partial $\left(C_{1}-C_{20}\right)$ SchulzFlory plots all of which are parabolic (so-called "double alpha") plots. Such double alpha plots have been observed before. A modified non-linear least squares method was used with the $\mathrm{C}_{1}$ $\mathrm{C}_{20}$ data to calculate values for alpha-1 and alpha- 2 as well as $\mathrm{XI}$ which is the carbon number "breakpoint": the carbon number at which two straight lines defined by the "linear" portions of the parabolic Schulz-Flory plot meet. The summary plots also include Figure A-20 which reports the weight percent of the total product in each of the historic fuel distillation ranges: gas $\left(C_{1}-C_{4}\right)$, gasoline $\left(C_{5}-C_{12}\right)$, diesel $\left(C_{13}-C_{19}\right)$ and heavy $\left(C_{20+}\right)$. The increase in light ends selectivity and the compensating decrease in wax yield due to the space velocity change are clearly visible in this plot.

\section{Run 43}

Potassium laurate was used again but in a lesser amount to provide a potassium level equal to that present in Run 37. Run 37 was the best previous run from the standpoint of low methane + ethane selectivity and high conversion. Although the iron-copper oxides used in Runs 37 and 42 were from different batches it was hoped that with similar potassium levels the catalyst performance during the two runs would be similar.

The initial operating conditions for this run were the same as Run 37; these conditions were maintained until an apparent line-out in conversions and selectivities was achieved. Plots of conversions and light product selectivities vs. hours-on-stream are attached as Figures A-21 to A-26. Line-out conversions and selectivities from Runs 37,42 and 43 are compared in Table 1. The CO conversion and methane/ethane selectivities for Run 43 were close to those of Run 37. It appears, therefore, that the simple addition of potassium laurate at the beginning of the run can replace the more time-consuming potassium carbonate impregnation with no ill effect to the catalyst.

During the latter part of this run space velocity and temperature changes were made to determine product selectivities at different conversion levels. The initial conditions were re-established at the end of the run to confirm that the catalyst had not changed in performance during operating condition excursions.

Analysis of the total liquid product was performed for three twenty-four hr periods (Period 4 , 72-96 hrs; Period 9, 192-216 hrs, and Period 15, 336-360 hrs). The Lotus spreadsheets which summarize these analyses are attached as Figures A-27 to A-29. The Schulz-Flory plots and the bar plot of gas, gasoline, etc. which were prepared from the spreadsheet data are attached as Figures A-30 to A-36.

Figure A-37 is a plot of autoclave liquid level vs. hours-on-stream. Except for a few times the liquid level was within one-half inch of the seven inch level target. 
The data from the condition changes are summarized in Figures A-38 to A-48. Two types of plots are represented in these Figures: selectivity vs. conversion and yield (selectivity times conversion) vs. contact time. The latter plots are helpful in determining which products are primary and which are secondary.

Figure A-38 is a selectivity vs. conversion plot for methane. The methane selectivity is greater at higher conversion presumably because the $\mathrm{H}_{2}$ : $\mathrm{CO}$ ratio increases as the conversion increases. Figure A-39 is a yield vs. contact time plot for methane, which shows methane formation at very short contact times. Methane, therefore, appears to be a primary product from the reaction of synthesis gas. There is no downward bend to the plot at long contact times which indicates that methane isn't converted into secondary products.

Figures A-40 to A-42 are yield vs. contact time plots for ethylene, ethane and ethanol. From these it appears that ethylene is a primary product which appears to be consumed at high contact times whereas ethane is a secondary product. The easiest guess as to the mechanism is that ethane results from hydrogenation of ethylene. Ethanol appears to be a primary product. (Yields for all the alcohols at the highest contact time are questionable because of the unstable alcohol selectivities at this condition-see alcohol selectivity vs. hours-on-stream plots in the Appendix.) Figures A-43 to A-48 are yield vs. contact time plots for $C_{3}$ and $C_{4}$ hydrocarbons and alcohols. Unlike ethane both propane and butane appear to be formed as primary reaction products. There is indication from the data at $255^{\circ} \mathrm{C}$ that propanol and butanol are formed as secondary reaction products.

\section{Conclusions}

A new, shorter, method for applying potassium to the iron-based Fischer-Tropsch catalyst has been developed. This method uses a potassium salt of a long-chain carboxylic acid which probably has enough solubility in the slurry oil present at start-up so that the potassium can be effectively transferred to the iron oxide catalyst. The resulting catalyst has nearly the same performance properties as one prepared by impregnation of potassium carbonate followed by calcination.

\section{Plans for the Next Quarter}

Additional experiments are contemplated with potassium laurate-derived catalysts. One specific approach will be to dissolve the potassium laurate in an organic solvent and add it to the slurry autoclave during a run. This might make it possible to adjust the conversion and/or selectivity during a run. 
TABLE 1

PERFORMANCE SUMMARY: TWO POTASSIUM ADDITION METHODS (LINED-OUT CATALYSTS)

\begin{tabular}{|c|c|c|c|}
\hline PLT/RUN NO. & $701 / 37$ & $701 / 42$ & $701 / 43$ \\
\hline K ADD. METHOD & $\mathrm{K}_{2} \mathrm{CO}_{3}$ IMPREG. & \multicolumn{2}{|c|}{$\begin{array}{c}\text { SOLID K LAURATE TO RX } \\
\text { AT STARTUP }\end{array}$} \\
\hline $\begin{array}{l}\text { CATALYST } \\
\text { OXIDE PART, } \\
\text { Fe, WT \% } \\
\text { K, WT \% } \\
\text { Fe:K, WT } \\
\text { K LAURATE, g } \\
\text { Fe:K, WT } \\
\text { (TOTAL) } \\
\end{array}$ & $\begin{array}{c}73 \\
64.2 \\
1.3 \\
100: 2.1 \\
100: 2.1\end{array}$ & $\begin{array}{c}73 \\
62.2 \\
0.0 \\
- \\
10 \\
100: 3.2\end{array}$ & $\begin{array}{c}73 \\
62.2 \\
0.0 \\
- \\
6.5 \\
100: 2.1\end{array}$ \\
\hline $\begin{array}{l}\text { TEST CONDS. } \\
\text { TEMP, }^{\circ} \mathrm{C} \\
\text { PRESS, PSIG } \\
\text { FEED RATE, } \\
(\mathrm{NL} / \mathrm{HR} \cdot \mathrm{g} \text { Fe) }\end{array}$ & $\begin{array}{l}265 \\
290 \\
2.4\end{array}$ & $\begin{array}{l}265 \\
290 \\
1.4\end{array}$ & $\begin{array}{l}265 \\
290 \\
2.4\end{array}$ \\
\hline $\begin{array}{l}\text { PERFORM. SUM. } \\
\text { CONVER., \% } \\
\text { CO } \\
\mathrm{H}_{2} \\
\text { PRODUCTIVITY } \\
\text { (M CO/HR.g AT. Fe) } \\
\text { SELEC., MOLE \% } \\
\mathrm{C}_{1} \\
\mathrm{C}_{2} \quad\left(\mathrm{C}_{1}+\mathrm{C}_{2}\right) \longrightarrow \\
\\
\mathrm{C}_{2}= \\
\mathrm{CO}_{2}\end{array}$ & $\begin{array}{l}3.8 \\
\frac{1.0}{4.8} \\
\\
3.0 \\
47 \\
\end{array}$ & $\begin{array}{l}2.5 \\
\frac{0.7}{3.2} \\
\\
2.5 \\
45 \\
\end{array}$ & $\begin{array}{l}4.1 \\
\frac{1.1}{5.2} \\
2.7 \\
47 \\
\end{array}$ \\
\hline
\end{tabular}

1. FROM $\mathrm{K}_{2} \mathrm{O}_{3}$ IMPREGNATION 


\section{POTTASIUM LAURATE ADDITION TO SLURRY \\ PIT 700B RUN $42 \quad \mathrm{H}_{2}: \mathrm{CO}$ foed $=0.7,1100 \mathrm{mpm}$}

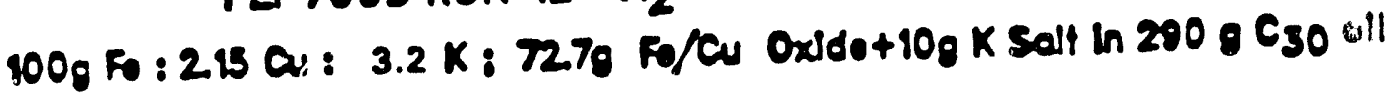

\begin{tabular}{l|l} 
TARGET TEMP, C & $280<$ \\
FEDD, NLM O FF & $2.0<$ \\
PRESSURE, PSIG & $253<$
\end{tabular}
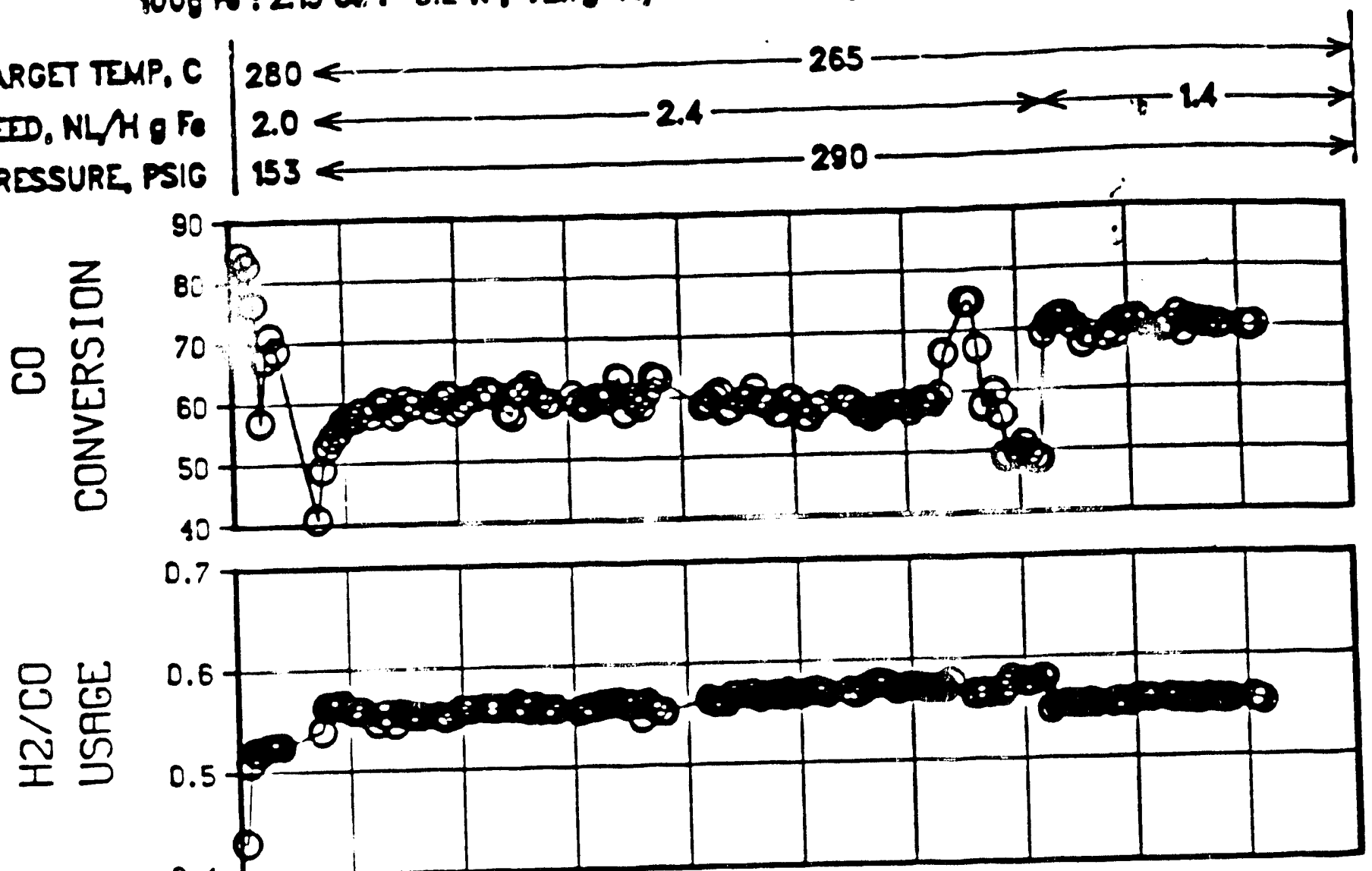

\section{4}

2.4 
FIGURE A-2

POTTASIUM LAURATE ADDITON TO SLURRY

PIJ $700 \mathrm{~B}$ RUN $42 \quad \mathrm{H}_{2}:$ :CO foed $=0.7,1100 \mathrm{rpm}$

$100 \mathrm{~g}$ Fo : 2.15 Cu: $3.2 \mathrm{~K}: 72.7 \mathrm{Fo} / \mathrm{Cu}$ Oxjdo+10g K Salt in 290 o C 30 oll

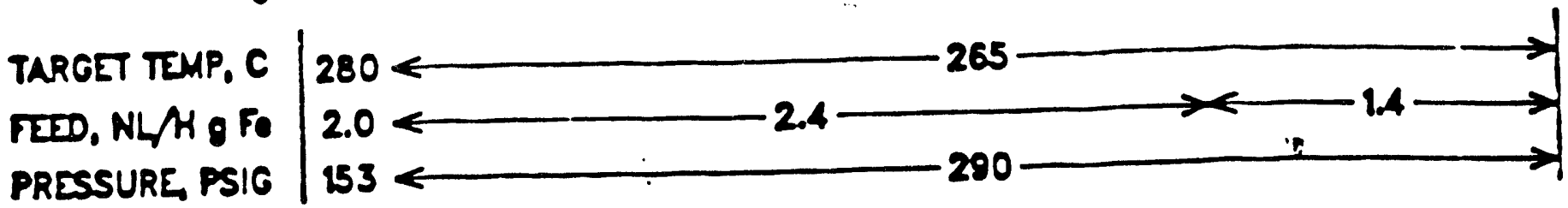

号岕
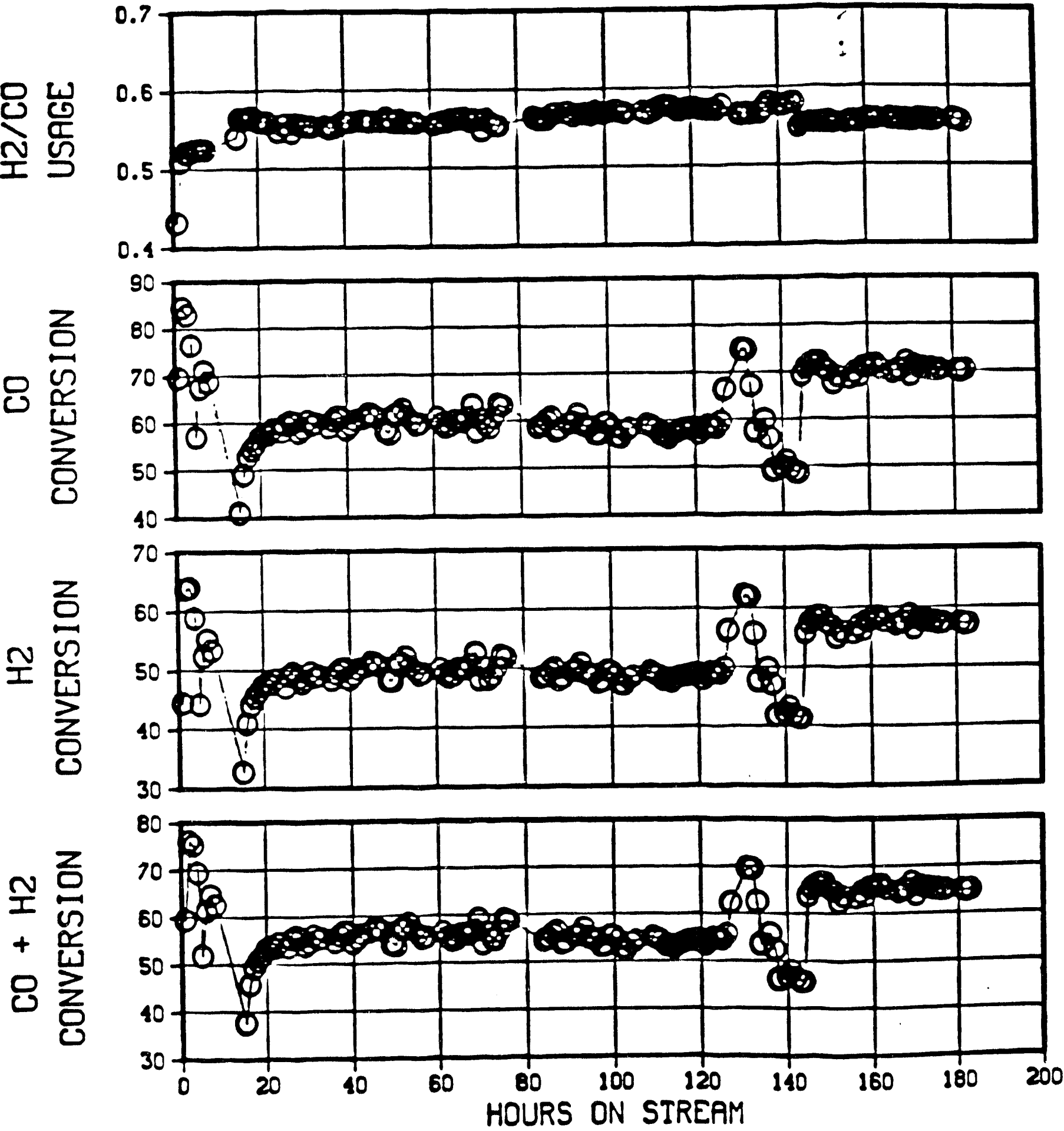


\section{POTTASIUM LAURATE ADDTION TO SLURRY}

\section{PLT 7008 RUN $42 \quad \mathrm{H}_{2}: \mathrm{CO}$ foed $=0.7,1100 \mathrm{rpm}$}

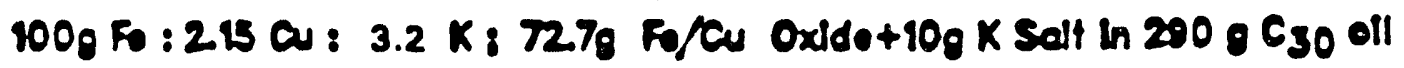
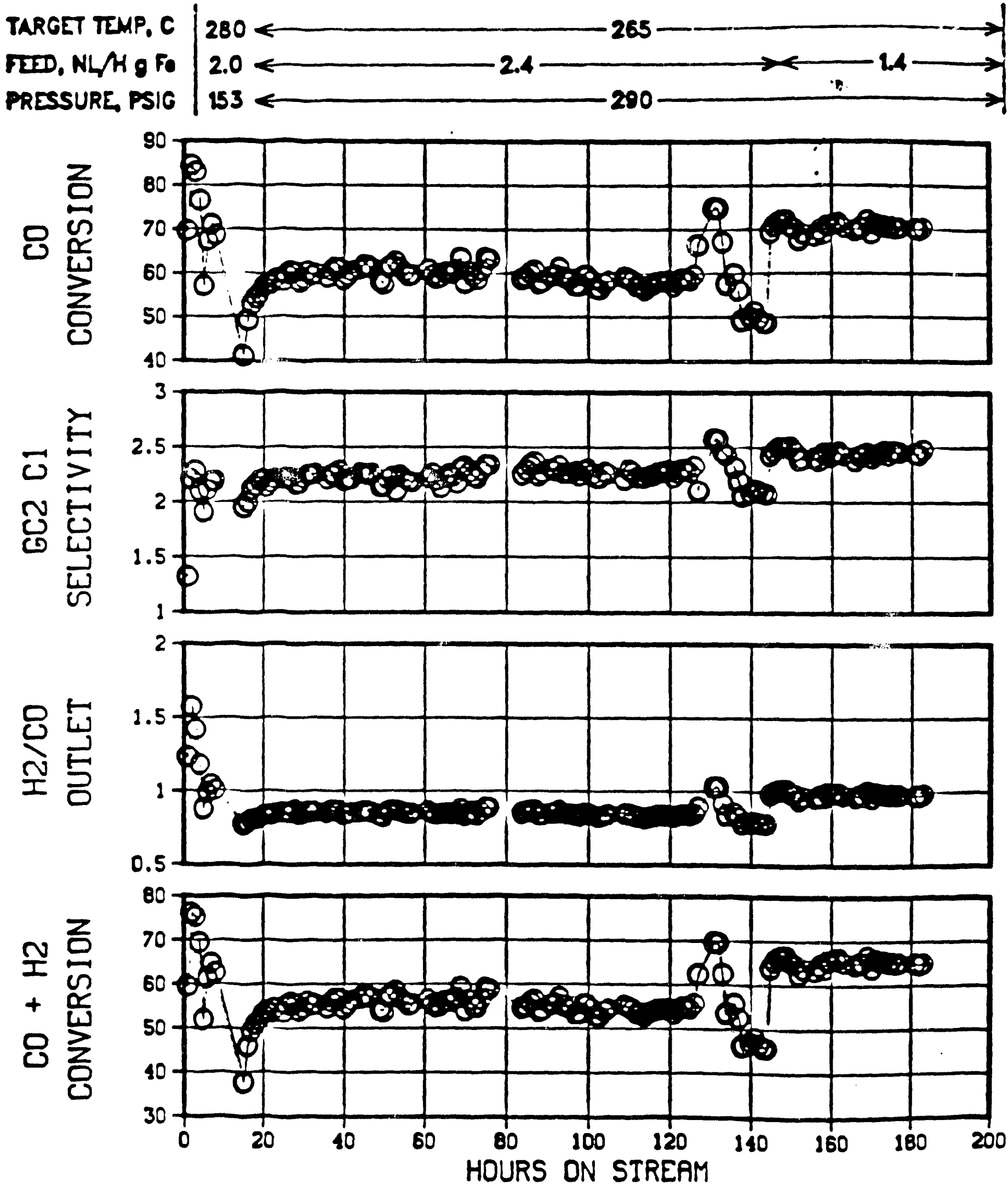


\section{FIGURE A-4 POTTASIUM LAURATE ADDTION TO SLURRY PLT 700B RUN $42 \quad \mathrm{H}_{2}: \mathrm{CO}$ food $=0.7,1100 \mathrm{rpm}$}

$100 \mathrm{~g}$ Fo : 2.15 Cu: $3.2 \mathrm{~K}: 72.70 \mathrm{Fo} / \mathrm{Cu}$ Oxidot $10 \mathrm{~g} \mathrm{~K} \operatorname{Salt}$ in $200, \mathrm{C}_{30}$ oll
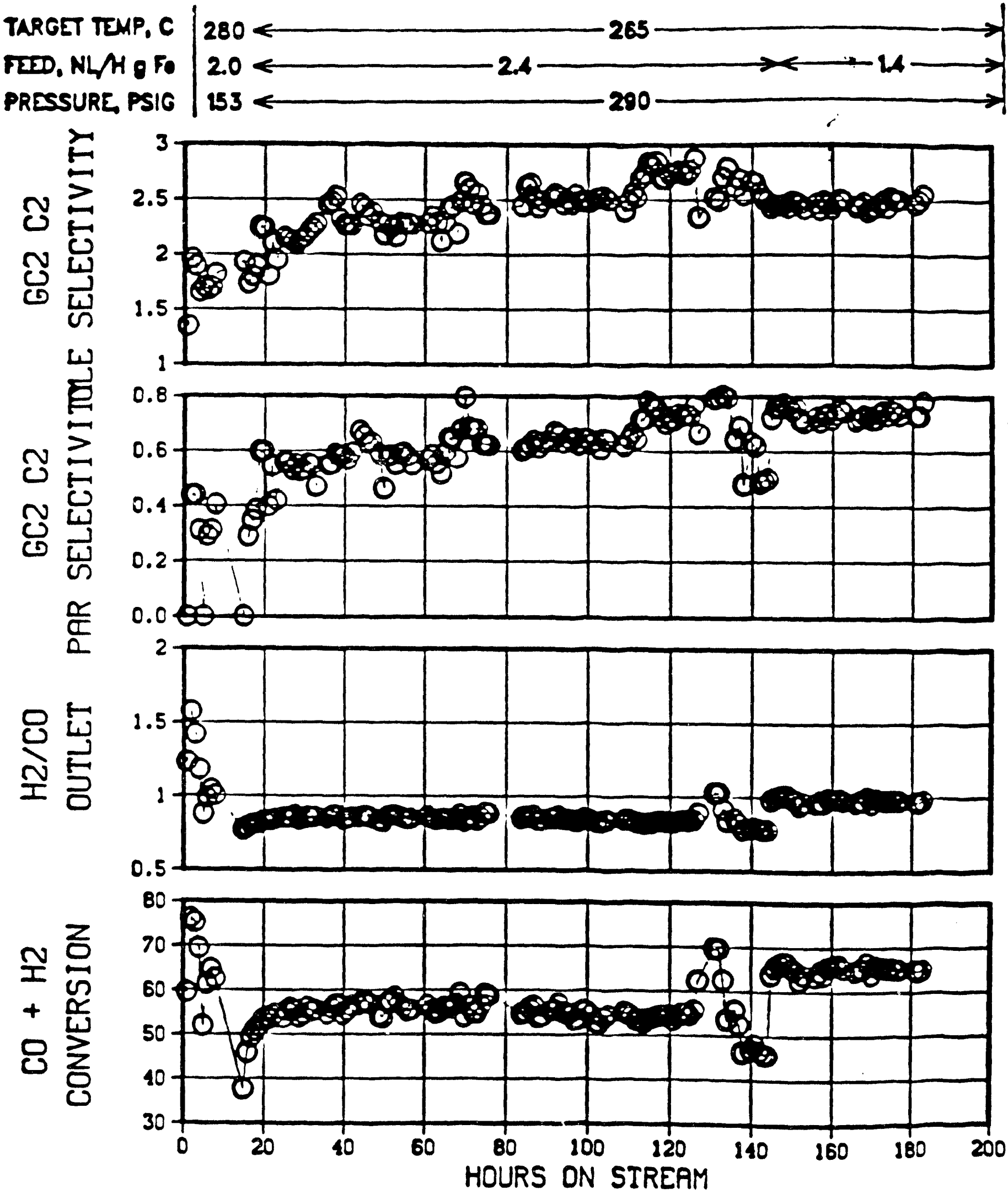


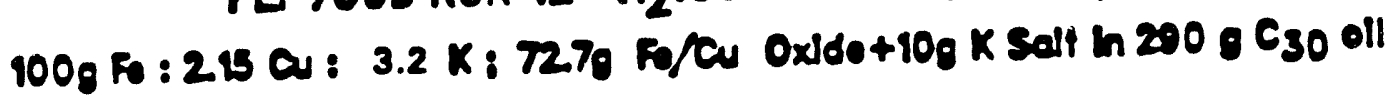

\begin{tabular}{l|l} 
TARGET TEMP, C & 280 \\
FEDD, NLM O FO & 2.0 \\
PRESSURE, PSIG & 253
\end{tabular}
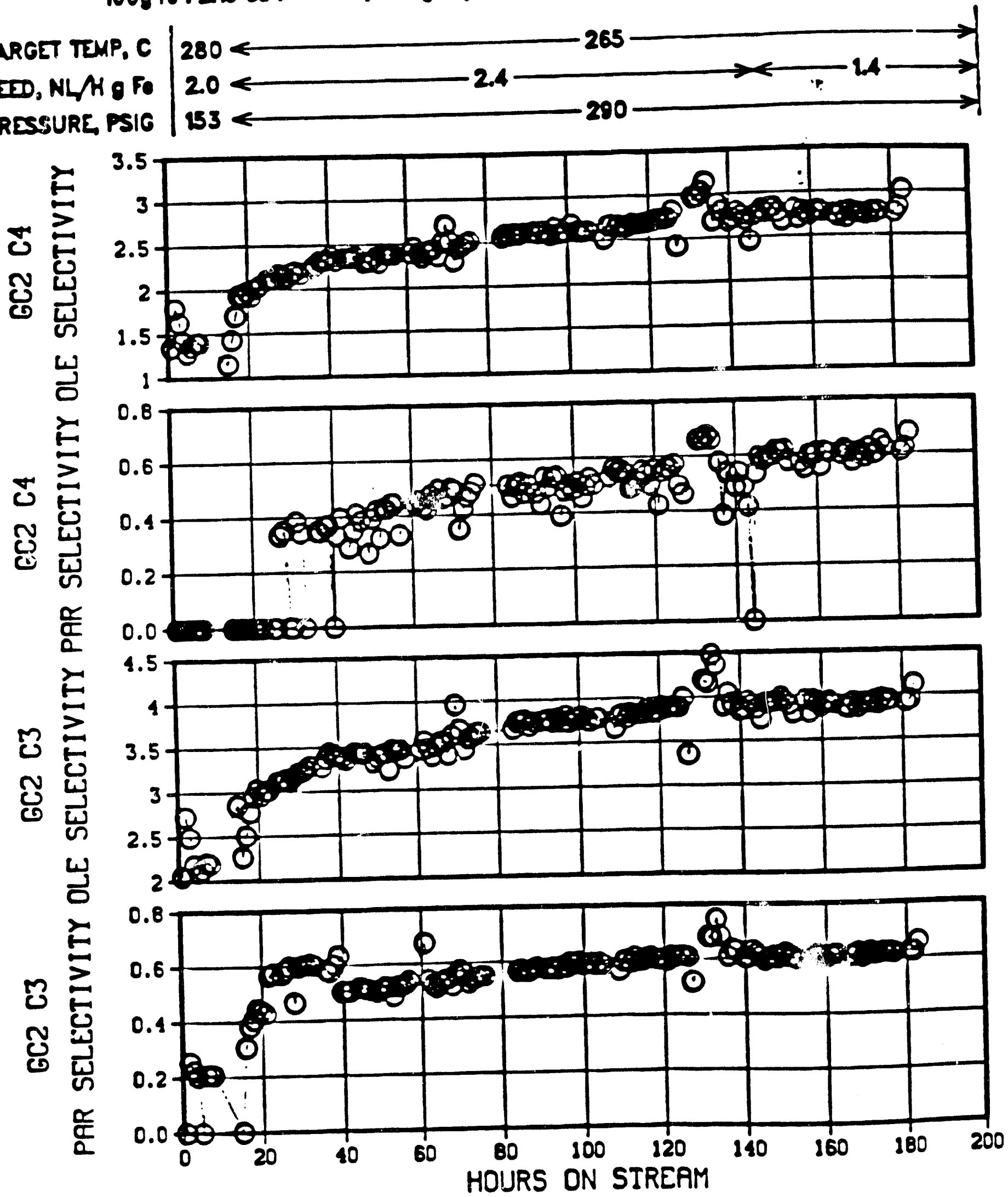
FIGURE A-6

\section{POTTASIUM LAURATE ADDTION TO SLURRY \\ PL 70OB RUN $42 \quad \mathrm{H}_{2}: C O$ fo.d $=0.7,1100 \mathrm{mpm}$}

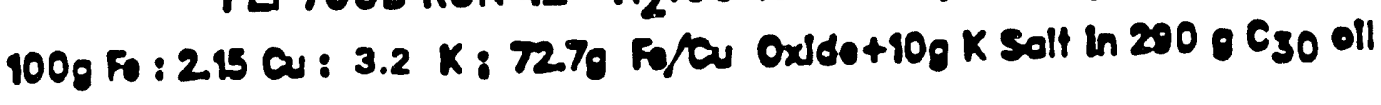
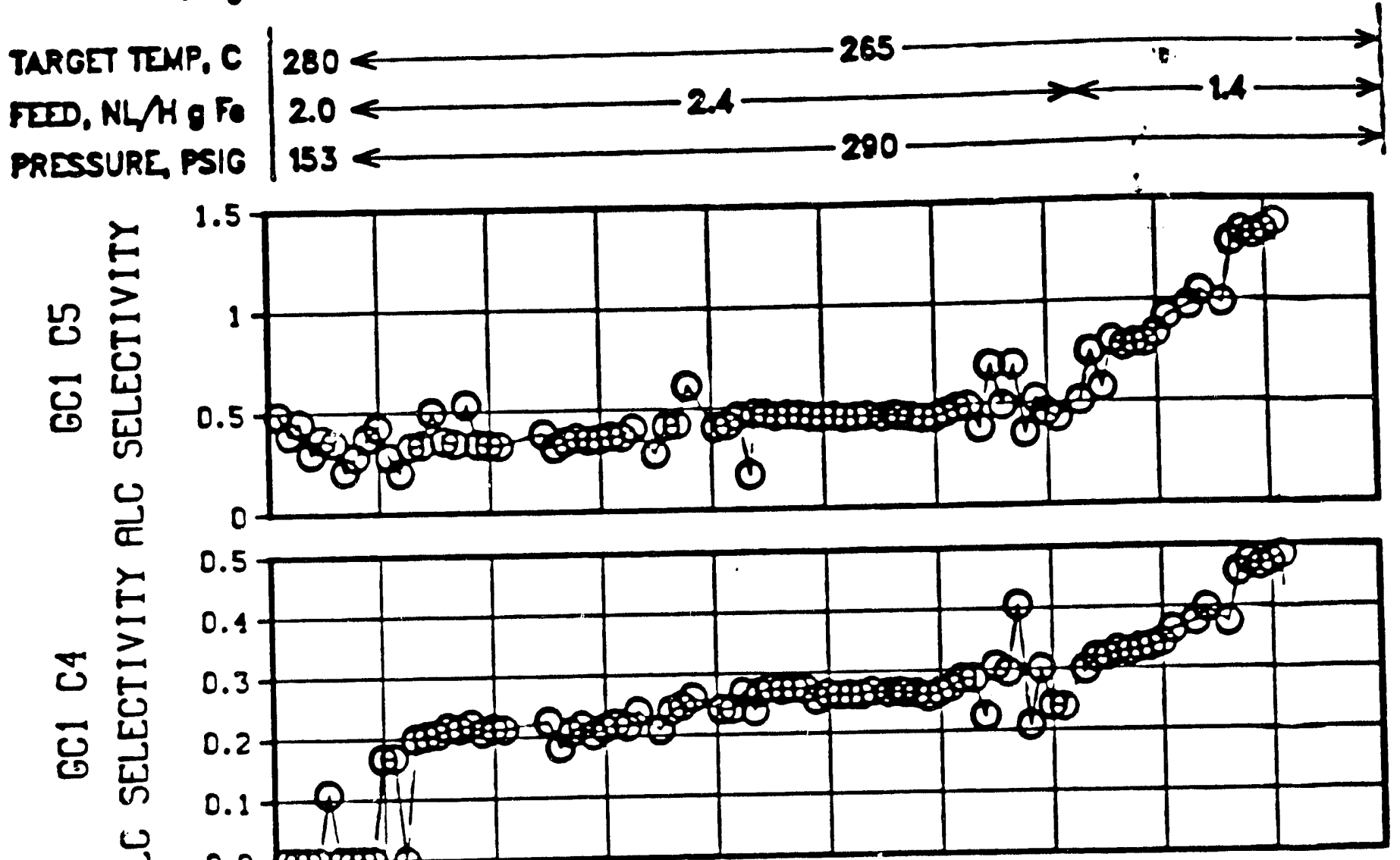

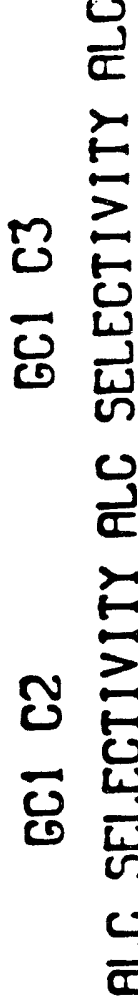

0.0
0.1

T

0.3

0.2

$0.1+\frac{108}{108}$

$0.0 \div 20$
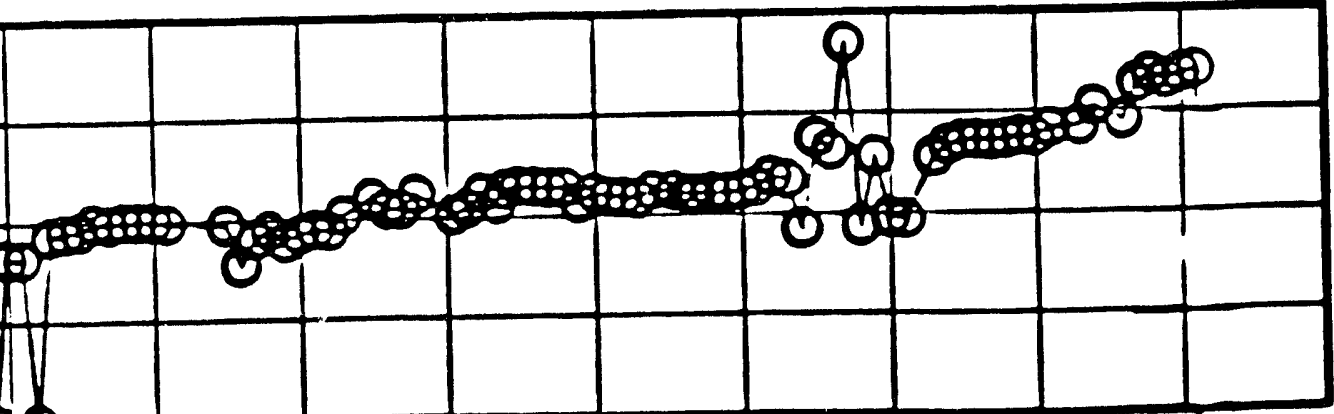

0.8

0.0
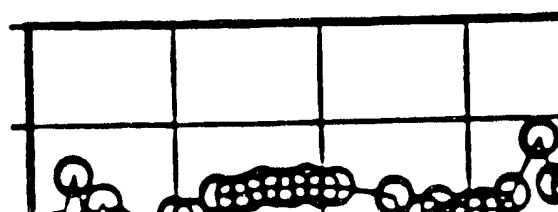

0.1 कि

0.2

0.0

0.0

0

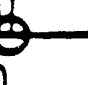


FIGURE A-7

- 20 HOURS ON STREAM

PLANT 700B RUN 42

口 - 50 HOURS ON STREAM

$\Delta-100$ HOURS ON STREAM

- -130 hOURS ON STREPH

$x-173$ hOURS ON STRERM

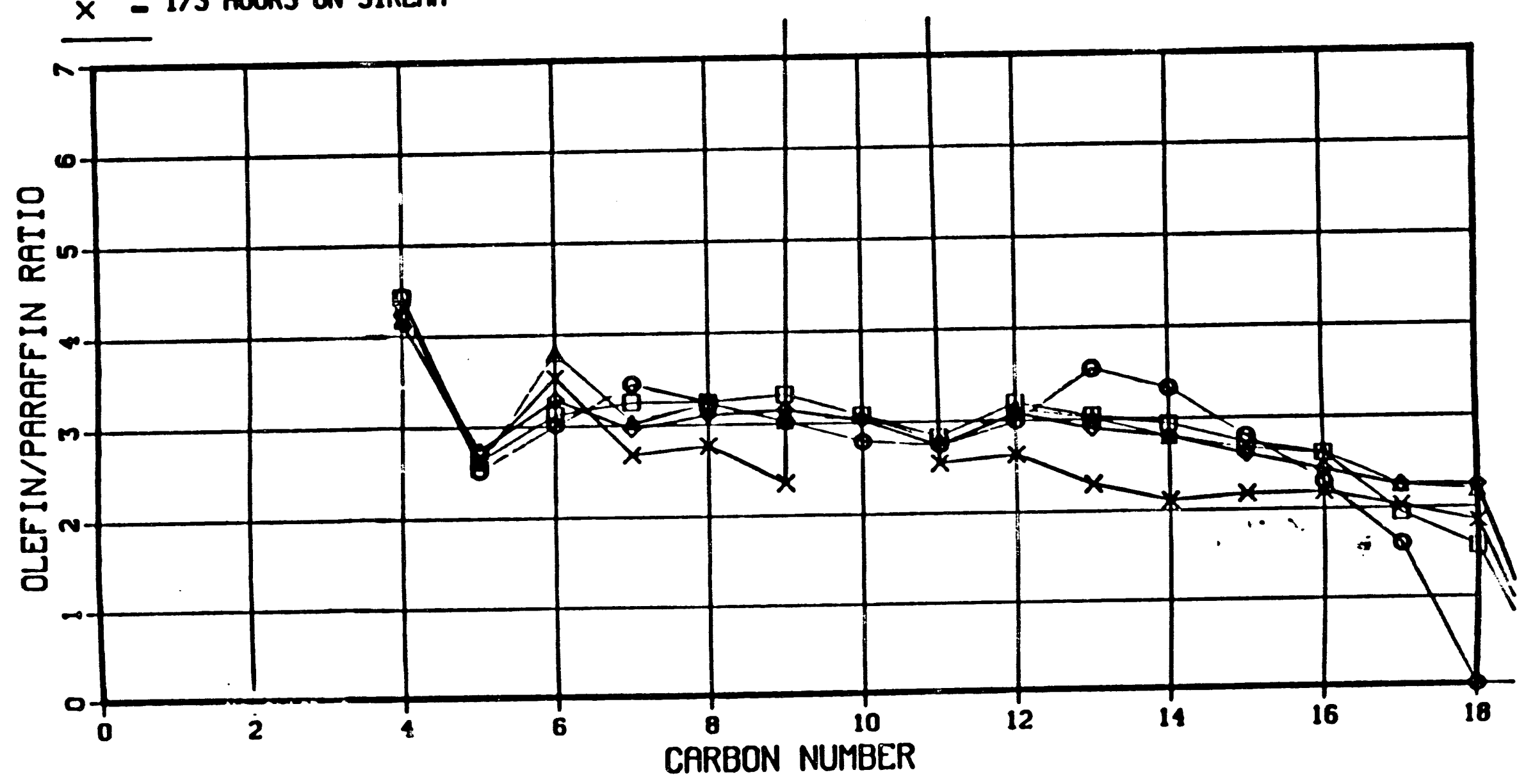




\section{\QUID LFVEL VS. HOURS ON STREAM PLANT 70OB RUN 42}

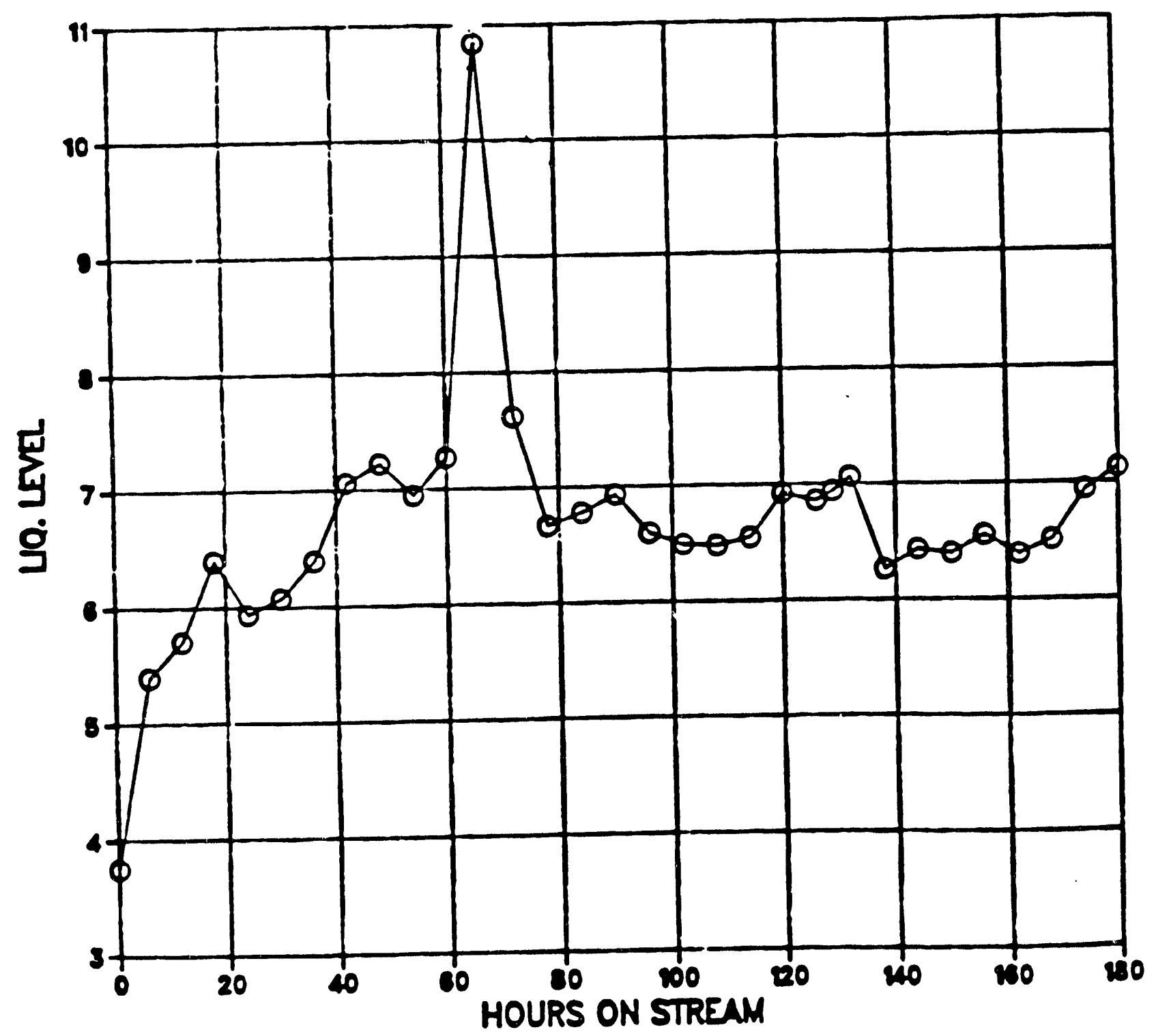


FIGURE A-9

CORRECTED WAX PRODUCT DISTRIBUTION

PLANT 700B, RUN 42, PERIOD 3

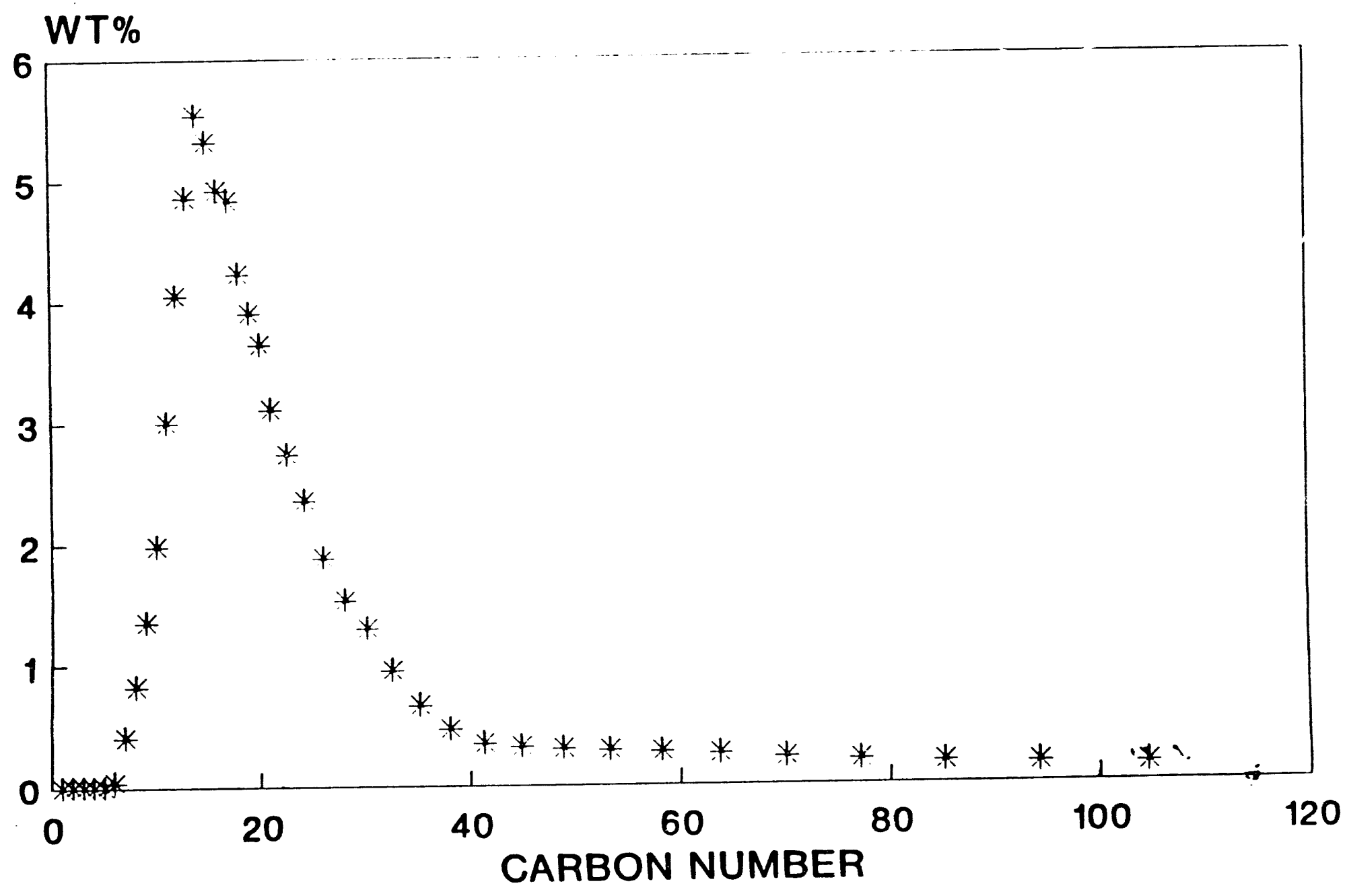

LIUIRUN42IP3-WAX2.CHT 
Piani 700E, RUN 12, PERJOL 3 TOTAL PRODUCTS

\begin{tabular}{|c|c|c|c|c|c|c|}
\hline C 1 & $\begin{array}{l}\text { MAX } \\
\text { nt? }\end{array}$ & $\begin{array}{c}\text { Max } \\
\text { Gran }\end{array}$ & $\begin{array}{r}\text { ICE AB } \\
\text { Grae }\end{array}$ & $\begin{array}{c}\text { ICE HC } \\
\text { Gran }\end{array}$ & $\begin{array}{c}\text { CRYO AO } \\
\text { Graa }\end{array}$ & $\begin{array}{r}\text { CYRO HC } \\
\text { Gra }\end{array}$ \\
\hline ta! & 100 & 62.6 & 0 & 25 & 0 & 3.03 \\
\hline 1 & 0 & 0.0810 & 0 & 0 & 0 & 0.037 \\
\hline 2 & 0 & 0.000 & 0 & 1.317 & 0 & 0.04 \\
\hline 3 & 0 & 0.000 & 0 & 1.581 & 0 & 0.241 \\
\hline 4 & 0 & 0.0010 & 0 & 2.838 & 0 & 0.563 \\
\hline
\end{tabular}

C5, C6 CORRECTED USINE

CS/CL, C6/CI RATIO BY GCI

GAS SUH

Gral Gras NTI: $\ln ($ (nTI/CN)

$23.67 \quad 121.98$

$3.56 \quad 5.60$

$6.16 \quad 7.52$

$8.31 \quad 10.13$

$3.64 \quad 9.04$ $\begin{array}{ll}4.59 & -3.082 \\ 6.16 & -3.480 \\ 8.31 & -3.587 \\ 7.41 & -3.989\end{array}$ 6A5

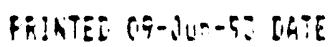

\begin{tabular}{|c|c|c|c|c|}
\hline 5 & 0 & 0.005 & 0 & 2.668 \\
\hline$t$ & 0.04 & 0.025 & 0 & $3.22 E$ \\
\hline 7 & 0.4 & 0.250 & 0 & 3.458 \\
\hline 8 & $0.8:$ & 0.543 & & 3.129 \\
\hline 9 & 1.35 & 0.845 & & $2.50 \mathrm{i}$ \\
\hline 10 & 1.52 & 1.239 & & 1.779 \\
\hline 11 & 3 & 1.878 & & 1.153 \\
\hline 12 & 4.05 & 2.535 & & 0.725 \\
\hline 13 & 4.86 & 3.012 & & 0.395 \\
\hline 14 & 5.54 & 3.468 & & 0.165 \\
\hline 15 & 5.32 & 3.330 & & 0 \\
\hline 16 & 1.92 & 3.080 & & 0 \\
\hline 17 & 4.84 & 3.030 & & 0 \\
\hline 18 & 4.23 & 2.648 & & \\
\hline 19 & 3.9 & 2.411 & & \\
\hline 20 & 3.64 & 2.279 & & \\
\hline 21.05 & 3.11 & 1.947 & & \\
\hline 22.6 & 2.73 & 1.709 & & \\
\hline 24.25 & 2.36 & 1.477 & & \\
\hline 26.05 & 1.88 & 1.177 & & \\
\hline 28.05 & 1.59 & 0.958 & & \\
\hline 30.2 & 1.29 & 0.808 & & \\
\hline 32.55 & 0.95 & 0.395 & & \\
\hline 35.2 & 0.66 & 0.413 & & \\
\hline 38.1 & 0.46 & 0.288 & & \\
\hline 41.3 & 0.34 & 0.213 & & \\
\hline 44.65 & 0.31 & 0.194 & & \\
\hline 48.8 & 0.29 & 0.182 & & \\
\hline 53.25 & 0.28 & 0.178 & & \\
\hline
\end{tabular}

$\begin{array}{rr}0 & 0.777 \\ 0 & 0.746 \\ & 0.359 \\ & 0.111 \\ & 0.071 \\ & 0.049 \\ & 0.033 \\ & 0 \\ & \\ & 0 \\ & 0 \\ & \\ & \end{array}$

$\begin{array}{lll}7.01 & 5.75 & -4.466 \\ 5.74 & 1.70 & -4.849 \\ 4.07 & 3.32 & -5.347 \\ 3.75 & 3.08 & -5.561 \\ 3.42 & 2.80 & -5.772 \\ 3.07 & 2.51 & -5.986 \\ 3.06 & 2.51 & -6.082 \\ 3.26 & 2.67 & -6.107\end{array}$

26.46

$\begin{array}{lll}3.44 & 2.82 & -6.134\end{array}$

$\begin{array}{lll}3.63 & 2.98 & -6.133\end{array}$

$\begin{array}{lll}3.33 & 2.73 & -6.309\end{array}$

$\begin{array}{lll}3.08 & 2.52 & -6.452\end{array}$

$\begin{array}{lll}3.03 & 2.48 & -6.529\end{array}$

$\begin{array}{lll}2.65 & 2.17 & -6.720\end{array}$

$2.44 \quad 2.00 \quad-6.836$

DIESEL

$\begin{array}{lll}2.28 & 1.87 & -6.976\end{array}$

$\begin{array}{lll}1.95 & 1.60 & -7.185\end{array}$

$\begin{array}{lll}1.71 & 1.40 \quad-7.386\end{array}$

$\begin{array}{lll}1.48 & 1.21 & -7.602\end{array}$

$\begin{array}{lll}1.18 & 0.96 & -7.901\end{array}$

$\begin{array}{lll}0.96 & 0.79 & -8.181\end{array}$

$\begin{array}{lll}0.81 & 0.66 & -8.125\end{array}$

$\begin{array}{lll}0.59 & 0.19 & -8.806\end{array}$

$\begin{array}{lll}0.41 & 0.34 & -9.249\end{array}$

$\begin{array}{lll}0.29 & 0.24 & -9.669\end{array}$

$\begin{array}{lll}0.21 & 0.17 & -10.072\end{array}$

$\begin{array}{lll}0.19 & 0.16 & -10.217\end{array}$

$\begin{array}{lll}0.18 & 0.15 & -10.398\end{array}$

$\begin{array}{lll}0.18 & 0.14 & -10.520\end{array}$

60.50LINE 27.36

17.71

MEAUY

28.46 


\section{FIGURE A-10 (CONT)}

\begin{tabular}{|c|c|c|}
\hline 63.79 & 0.29 & 0.13 \\
\hline 70.05 & 0.22 & 0.13 \\
\hline 77.13 & 0.15 & 0.119 \\
\hline Es.ds & 0.17 & 0.10 \\
\hline 94.25 & 0.16 & 0.10 \\
\hline 104.35 & i..14 & 0.08 \\
\hline 120 & 0.07 & 0. \\
\hline 134 & 0.27 & 0.1 \\
\hline 146 & $0.0 E$ & 0.0 \\
\hline 16.4 & 6.0. & \\
\hline $165 . .5$ & 6.64 & 次 \\
\hline$\because \because 8$ & 0,83 & $\therefore$ \\
\hline QSE.8 & 0.02 & 0.0 \\
\hline 29.28 & 0.61 & .8 \\
\hline $9: 2.8$ & C.id & \\
\hline & ( & \\
\hline
\end{tabular}

\begin{tabular}{|c|c|c|}
\hline v.10 & vols i & \\
\hline 0.14 & 0.11 & -11.056 \\
\hline 0.22 & $0.10^{\circ}$ & -11.275 \\
\hline 0.11 & 0.09 & -11.489 \\
\hline 0.10 & 0.08 & -11.651 \\
\hline 0.07 & 0.07 & -11.688 \\
\hline 0.04 & 0.04 & -12.719 \\
\hline 0.11 & 0.09 & $-! 1.942$ \\
\hline 0.05 & 0.18 & $-\therefore . .7 E 2$ \\
\hline 0.016 & $0.0:$ & $-12.18 t$ \\
\hline 0.03 & 0.02 & -83.712 \\
\hline C.6: & 0.02 & $-14.22 t$ \\
\hline 0.11 & 0.31 & -14.659 \\
\hline $0.0:$ & 0.01 & -25.425 \\
\hline 0.01 & O.C: & $-: 3.6: 29$ \\
\hline 0.1 & C.r: & EF \\
\hline
\end{tabular}

Sl: t. t. $.9 ?$ \$1.99:3\%

$0 \quad 24.95 .6$

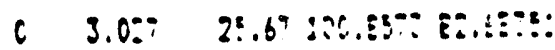




\section{FIGURE A-11}

PLANT 7008, RUH 12, PERI2O 6 TCTAL PRODUCTS

\begin{tabular}{|c|c|c|c|c|c|c|c|c|c|c|}
\hline C 1 & $\begin{array}{l}\text { Mex } \\
\text { wtI }\end{array}$ & $\begin{array}{l}\text { MAX } \\
\text { Gras }\end{array}$ & $\begin{array}{r}\text { ICE } 6.0 \\
6 \mathrm{rat}\end{array}$ & $\begin{array}{r}\text { ICE HC } \\
\text { Gras }\end{array}$ & $\begin{array}{r}\text { CRYO AO } \\
\text { GPAO }\end{array}$ & $\begin{array}{r}\text { EYRO HC } \\
\text { GPae }\end{array}$ & $\begin{array}{l}\text { 6A5 } \\
\text { Gran }\end{array}$ & $\begin{array}{l}\text { SUM } \\
\text { Gras }\end{array}$ & UT\% & $\ln (N i z, C x ;$ \\
\hline ictal & 100 & $16 t$ & 0 & 28.99 & 0 & 13.98 & 29.66 & 248.94 & & \\
\hline 1 & 0 & 0.000 & 0 & 0 & 0 & 0 & 5.64 & 3.64 & 2.27 & -3.737 \\
\hline 2 & 0 & 0.000 & 0 & 0.572 & 0 & 0.322 & 7.59 & 8.44 & 3.39 & -4.677 \\
\hline 3 & 0 & 0.000 & 0 & 1.1 & 0 & 0.254 & 9.6 & 10.98 & 4.41 & -1.219 \\
\hline 4 & 0 & 0.000 & 0 & 1.84 & 0 & 0.568 & 6.87 & 9.28 & 3.73 & -4.576 \\
\hline 5 & 0 & 0.090 & 0 & 2.2 & 0 & 0.644 & & 9.96 & 3.96 & -4.238 \\
\hline 6 & 0.04 & 0.066 & 0 & 2.74 & 0 & 2.76 & & 8.85 & 3.56 & -5.127 \\
\hline 7 & 0.24 & 0.398 & 0 & 3.84 & & 3.48 & & 7.72 & 3.10 & -5.120 \\
\hline 6 & 0.29 & 0.481 & & 4.33 & & 2.59 & & 7.40 & 2.97 & -5.595 \\
\hline 9 & 0.59 & 0.979 & & 3.88 & & 1.69 & & $6.5 i$ & 2.61 & $-9.64 !$ \\
\hline$: 0$ & 0.94 & 1.560 & & 2.94 & & 0.785 & & 5.47 & 2.20 & $-6.11 ! 8$ \\
\hline 11 & 2.28 & 2.325 & & 2.08 & & 0.474 & & 4.68 & 1.38 & -6.372 \\
\hline 12 & 1.66 & 2.756 & & 1.47 & & 0.17 & & 4.40 & 1.77 & -6.522 \\
\hline 13 & 1.86 & 3.058 & & 0.198 & & 0.057 & & 4.04 & 1.62 & $-5.6 E j$ \\
\hline 14 & 2.12 & 3.519 & & 0.49 & & 0 & & 4.01 & 1.61 & -6.768 \\
\hline 15 & 2.11 & 3.503 & & 0.286 & & 0 & & 3.79 & 1.52 & -6.893 \\
\hline 16 & 2.03 & 3.370 & & 0.122 & & & & 3.49 & $1.40^{\circ}$ & -7.039 \\
\hline 17 & 2.07 & 3.436 & & 0.082 & & & & 3.52 & 1.41 & -7.092 \\
\hline 18 & 1.88 & 3.121 & & 0.092 & & & & 3.20 & 1.29 & -7.244 \\
\hline 19 & 1.8 & 2.988 & & 0.041 & & & & 3.03 & 1.22 & -7.353 \\
\hline 20 & 1.73 & 2.872 & & & & & & 2.87 & 1.15 & -7.458 \\
\hline 21.05 & 1.52 & 2.323 & & & & & & 2.32 & 1.01 & -7.639 \\
\hline 22.6 & 1.44 & 2.390 & & & & & & 2.39 & 0.96 & -7.764 \\
\hline 24.25 & 1.37 & 2.274 & & & & & & 2.27 & 0.91 & -7.884 \\
\hline 26.05 & 1.24 & 2.058 & & & & & & 2.06 & 0.83 & -8.055 \\
\hline 28.05 & 1.13 & 1.676 & & & & & & 1.88 & 0.75 & -8.222 \\
\hline
\end{tabular}

C5 CORRECTED USINS

CS/CL RATIO BY SCI 


\section{FIGURE A-11 (CONT)}

\begin{tabular}{|c|c|c|}
\hline 36.2 & 1.19 & 1.809 \\
\hline 32.95 & 0.59 & $1.64 ;$ \\
\hline 35.2 & 0.90 & 1.494 \\
\hline $3 E .1$ & 0.86 & 1.428 \\
\hline 4i.3 & 0.80 & .328 \\
\hline 44.8: & 0.76 & $.2 t 2$ \\
\hline AE. 6 & .72 & $1 .: 95$ \\
\hline 83.29 & 0.68 & 1.129 \\
\hline 5.2 & 0.65 & 1.079 \\
\hline$t \therefore .75$ & 0.61 & 1.023 \\
\hline 70.15 & 0.5 & $0.94 E$ \\
\hline 97.18 & 0.35 & $6.85:$ \\
\hline E!...! & $(1,69$ & .812 \\
\hline $94,8:$ & 0.45 & 0.714 \\
\hline 104.88 & 0.38 & $0.63:$ \\
\hline $10 \hat{0}$ & $0.2 i$ & $0.3: 2$ \\
\hline di: & $0.4 t$ & 0.704 \\
\hline 16E & 0.20 & 0.332 \\
\hline $10 t$ & $0 .: 3$ & 0.236 \\
\hline $16 ? .9$ & 0.19 & $0.14 ;$ \\
\hline 8:6 & 0.06 & 0.100 \\
\hline ZiE.5 & 8.6? & 0.050 \\
\hline$\because \because .5$ & C.0! & 0.017 \\
\hline 319.5 & 0.01 & $0.0 j 9$ \\
\hline 39 & . & 0.006 \\
\hline
\end{tabular}

\begin{tabular}{|c|c|c|}
\hline 1.81 & $\begin{array}{l}0.73^{\circ}- \\
0.66\end{array}$ & $\begin{array}{l}-1.332 \\
-8.303\end{array}$ \\
\hline $\begin{array}{l}1.04 \\
1.49\end{array}$ & 0.60 & -8.677 \\
\hline 1.43 & 0.57 & -8.801 \\
\hline 1.33 & 0.53 & -8.994 \\
\hline 1.26 & 0.31 & -9.088 \\
\hline 1.20 & 0.48 & -9.227 \\
\hline 1.13 & 0.45 & -9.371 \\
\hline 1.08 & 0.43 & -9.505 \\
\hline 01 & $0.4 !$ & -9.650 \\
\hline 99 & 0.38 & -9.822 \\
\hline$B E$ & 0.35 & -9.991 \\
\hline.$E 1$ & 0.23 & -10.168 \\
\hline 71 & 0.29 & -10.460 \\
\hline 0.63 & 0.25 & $-10 . \leqslant 2 E$ \\
\hline 0.33 & 0.13 & -11.407 \\
\hline 0.76 & 0.31 & -10.689 \\
\hline 0.33 & 0.13 & -11.603 \\
\hline 0.22 & 0.08 & -12.130 \\
\hline 0.13 & 0.06 & -12.641 \\
\hline 0.10 & 0.04 & -13.171 \\
\hline 0.05 & 0.02 & $-13.99:$ \\
\hline 0.02 & 0.01 & -15.223 \\
\hline 0.02 & 0.01 & -19.360 \\
\hline 0.0 & 0.00 & ER \\
\hline
\end{tabular}




\section{FIGURE A-12}

PLANT 7008, RUN 42, PERIOD B TOTAL PROIUJCTS

\begin{tabular}{|c|c|c|c|c|c|c|c|c|c|c|}
\hline$C 1$ & $\begin{array}{l}\text { WAX } \\
\text { wty }\end{array}$ & $\begin{array}{l}\text { WAX } \\
\text { Gras }\end{array}$ & $\begin{array}{r}\text { ICE AO } \\
\text { bras }\end{array}$ & $\begin{array}{l}\text { ICE HC } \\
\text { GraA }\end{array}$ & $\begin{array}{r}\text { CRYC AD } \\
\text { GraA }\end{array}$ & $\begin{array}{r}\text { CYKO HC } \\
\text { GPAS }\end{array}$ & $\begin{array}{l}\text { 6AS } \\
\text { 6ran }\end{array}$ & $\begin{array}{l}\text { SUH } \\
\text { Sras }\end{array}$ & Nit & $\ln (H T Z / C N)$ \\
\hline Total & 100 & 61 & 0 & 22.99 & 0 & 13 & 19.17 & 116.16 & & \\
\hline 1 & 0 & 0.000 & 0 & 0 & 0 & 0 & 3.89 & 3.88 & 3.34 & -3.399 \\
\hline 2 & 0 & 0.000 & 0 & 0.6 & 0 & 0.655 & 4.45 & 3.71 & 4.91 & -3.70 .7 \\
\hline 3 & 0 & 0.000 & 0 & 0.979 & 0 & 0.638 & 6.27 & 7.89 & 6.79 & -3.788 \\
\hline 4 & 0 & 0.000 & 0 & 1.64 & 0 & 1.621 & 4.37 & 7.83 & 6.74 & -4.083 \\
\hline 5 & 0 & 0.000 & 0 & 1.93 & 0 & 2.8 & & 4.73 & 4.07 & $-4.8: 0$ \\
\hline 6 & 0.04 & 0.024 & 0 & 2.56 & 0 & 3 & & 3.53 & 4.81 & -4.827 \\
\hline 7 & 0.22 & 0.134 & 0 & 3.32 & & 2.03 & & 3.48 & 4,72 & -4.999 \\
\hline$B$ & 0.27 & 0.165 & & 3.47 & & 1.13 & & 4.76 & 4.10 & -5.273 \\
\hline 9 & 0.59 & 0.360 & & 2.97 & & 0.573 & & 3.90 & 3.36 & -5.590 \\
\hline
\end{tabular}


FIGURE A-12 (CONT)

\begin{tabular}{|c|c|c|c|c|c|c|c|c|}
\hline \multirow[b]{2}{*}{10} & \multirow[b]{2}{*}{1.07} & \multirow[b]{2}{*}{0.693} & \multirow[b]{2}{*}{2.18} & \multirow[b]{2}{*}{0.327} & \multicolumn{4}{|c|}{ 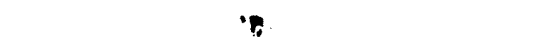 } \\
\hline & & & & & 3.16 & 2.72 & -9.907 & \\
\hline 11 & 1.24 & 0.756 & 1.45 & 0.147 & 2.35 & $2.03 j$ & -6.297 & \\
\hline 12 & 1.3 & 0.793 & 0.916 & 0.065 & 1.77 & $1.33^{\circ}$ & $-6.667 \mathrm{SA}$ & $\begin{array}{r}\text { ASOLITEE } \\
27.34\end{array}$ \\
\hline 13 & 1.29 & 0.787 & 0.442 & 0.016 & 1.24 & 1.07 & -7.101 & \\
\hline 14 & 1.37 & 0.836 & 0.221 & 0 & 1.06 & 0.91 & -7.339 & \\
\hline 15 & 1.28 & 0.781 & 0.126 & 0 & 0.91 & 0.78 & $-7.56 i$ & \\
\hline db & 1.23 & 0.790 & 0.095 & & 0.83 & 0.73 & -7.696 & \\
\hline 17 & 1.23 & 0.750 & 0.063 & & 0.81 & 0.70 & -7.799 & \\
\hline 16 & 1.17 & 0.714 & 0.031 & & 0.74 & 0.64 & -7.940 & \\
\hline 19 & 1.8 & $0.79: 2$ & 0 & & 0.73 & 0.63 & -8.011 & $\begin{array}{r}\text { DIESE! } \\
3.46\end{array}$ \\
\hline 20 & 1.25 & $0.76 ?$ & 0 & & 0.76 & 0.66 & -8.022 & \\
\hline al.r: & 1.17 & 0.714 & & & 0.71 & 0.61 & -8.139 & REAUY \\
\hline 22.6 & 1.16 & 0.708 & & & 0.71 & 0.61 & -8.219 & 45.42 \\
\hline 24.25 & 1.14 & 0.695 & & & 0.70 & 0.60 & -8.307 & \\
\hline 26.05 & d.ng & $0.64 ?$ & & & 0.65 & 0.56 & -8.451 & \\
\hline 28.05 & 1.01 & 0.616 & & & 0.62 & 0.33 & -8.573 & \\
\hline 30.2 & 2.01 & 0.616 & & & 0.62 & 0.53 & -8.647 & \\
\hline 32.85 & 0.94 & 0.573 & & & 0.57 & 0.49 & -8.794 & \\
\hline 35.2 & 0.86 & 9.525 & & & 0.32 & 0.45 & -8.961 & \\
\hline 38.1 & 0.83 & 0.5 it & & & 0.91 & 0.44 & -9.076 & \\
\hline 41.3 & 0.79 & $0.48 \%$ & & & 0.48 & 0.41 & -9.206 & \\
\hline 44,89 & 0.75 & $0.43 E$ & & & 0.46 & 0.39 & -9.340 & \\
\hline 48.8 & 0.90 & 0.427 & & & 0.43 & 0.37 & -9.494 & \\
\hline 53.25 & 0.66 & 0.463 & & & 0.40 & 0.35 & -9.640 & \\
\hline 38.2 & $0.6 ?$ & 0.354 & & & 0.38 & 0.33 & -9.778 & \\
\hline 63.75 & 0.59 & $0.3 e s$ & & & 0.36 & 0.31 & -9.932 & \\
\hline 70.05 & 0.94 & 0.329 & & & 0.33 & 0.28 & -10.115 & \\
\hline 79.15 & 0.52 & $0.3: 7$ & & & 0.32 & 0.27 & -10.249 & \\
\hline 89.19 & $0.4 ?$ & 0.287 & & & 0.29 & 0.23 & -10.449 & \\
\hline 94.33 & $0.4 j$ & 0.262 & & & 0.26 & 0.23 & -10.639 & \\
\hline 184.59 & 0.40 & 0.244 & & & 0.24 & 0.21 & -10.815 & \\
\hline 120 & 0.22 & 0.134 & & & 0.13 & 0.12 & -21.551 & \\
\hline 134 & 0.35 & 0.336 & & & 0.34 & 0.29 & -10.745 & \\
\hline 116 & 0.27 & 0.169 & & & 0.16 & 0.14 & -11.542 & \\
\hline 164 & 0.20 & 0.122 & & & 0.12 & 0.11 & -11.959 & \\
\hline 185.3 & 0.13 & 0.092 & & & 0.09 & 0.08 & -12.369 & \\
\hline 210 & 0.80 & 0.061 & & & 0.06 & 0.05 & -12.099 & \\
\hline 238.5 & 0.06 & 0.037 & & & 0.04 & 0.03 & -13.337 & \\
\hline $27 \vdots .5$ & 0.04 & 0.024 & & & 0.02 & 0.02 & -11.076 & \\
\hline 312.5 & 0.02 & 0.012 & & & 0.01 & 0.01 & -11.906 & $\cdot$ \\
\hline 359 & 0.01 & 0.006 & & & 0.01 & 0.01 & -19.738 & \\
\hline
\end{tabular}

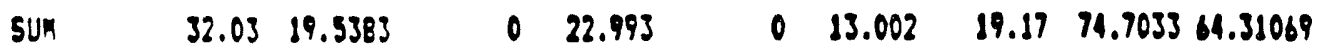


FIGURE A-13

TOTAL HC PRODUCT DISTRIBUTION

PLANT 700B, RUN 42

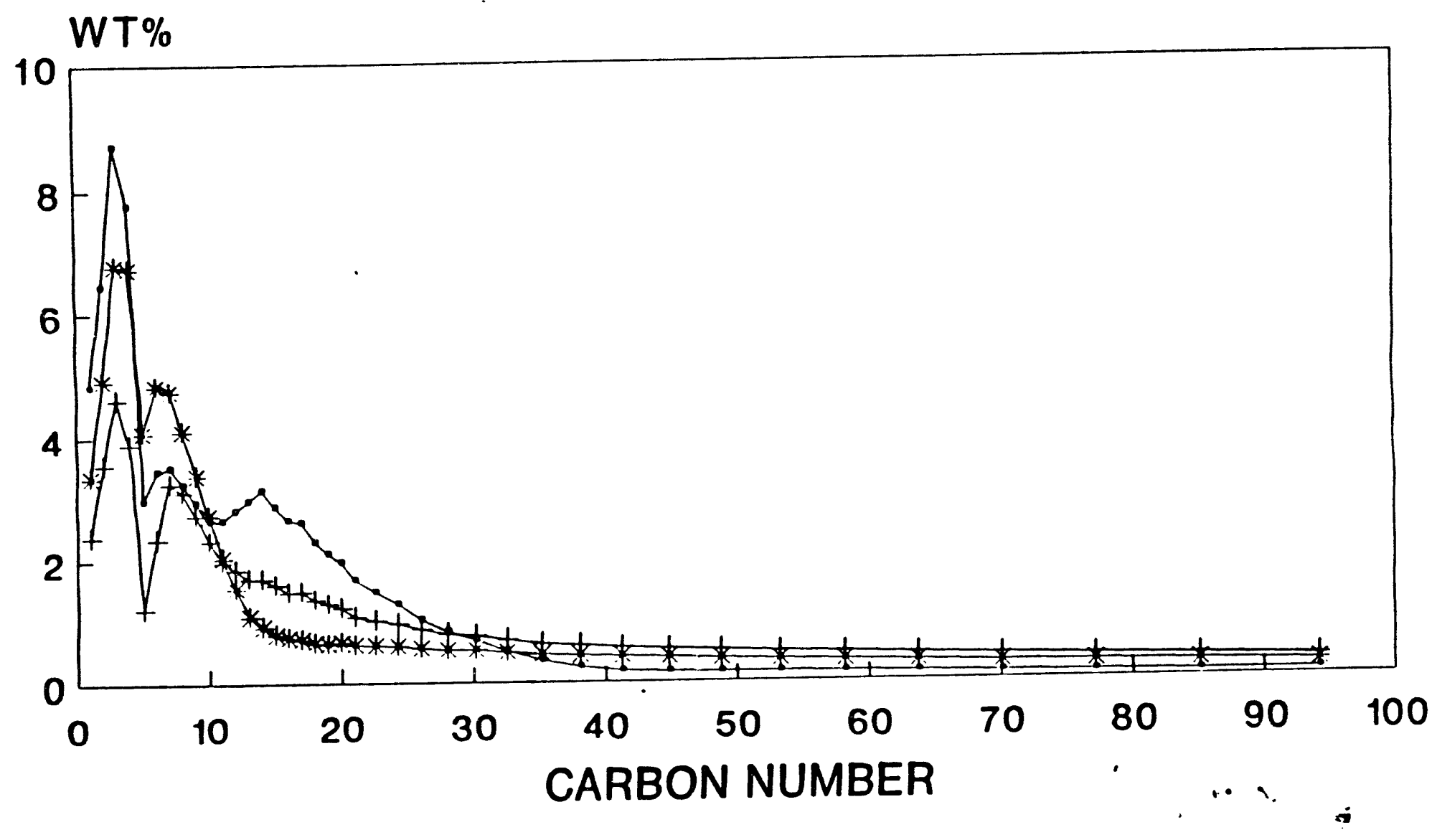

- PERIOD $3 \rightarrow$ PERIOD 6 * PERIOD 8 


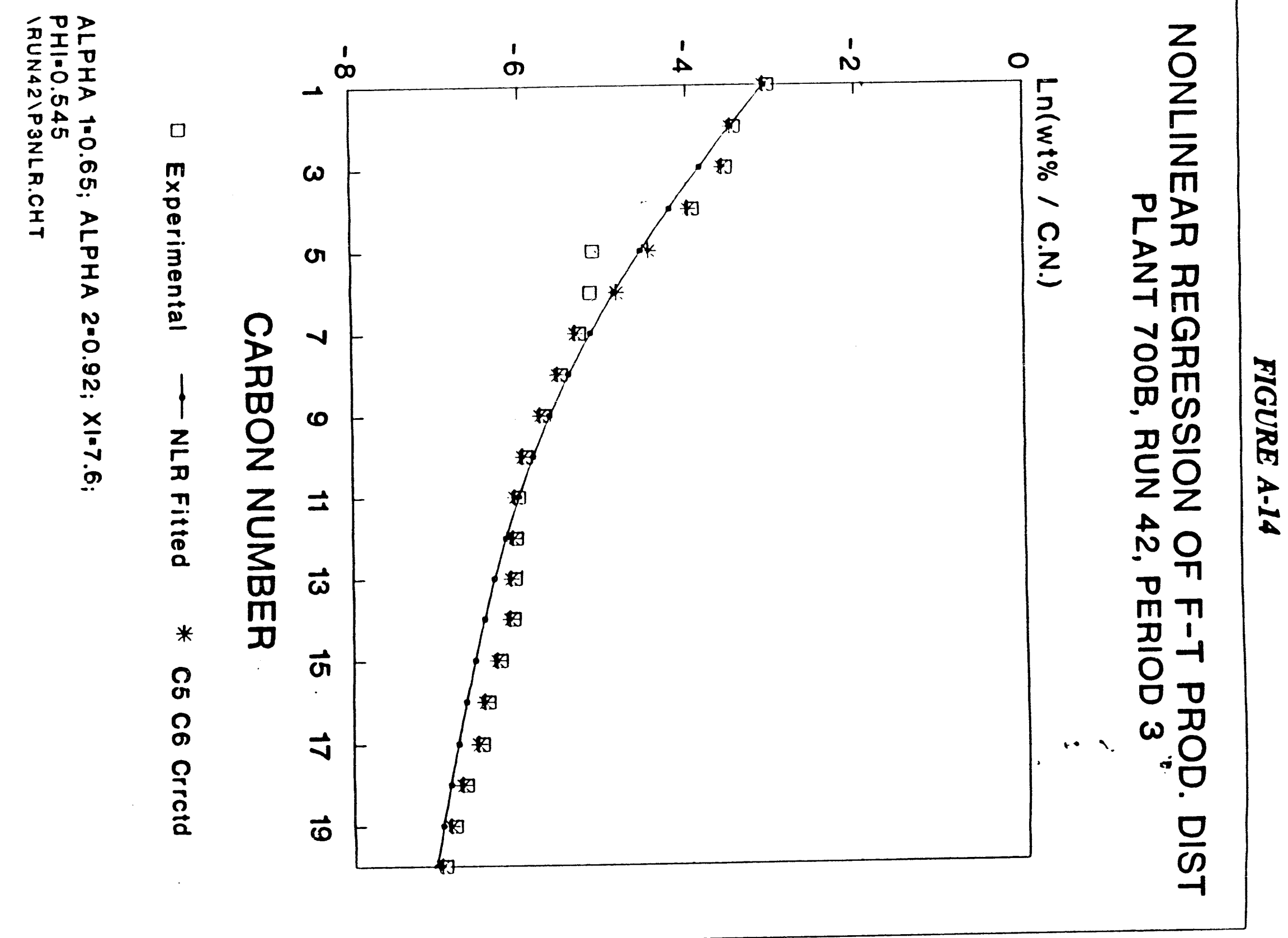


FIGURE A-15

\section{SCHULZ-FLORY DISTRIBUTION \\ PLANT 700B, RUN 42, PERIOD 3 '}

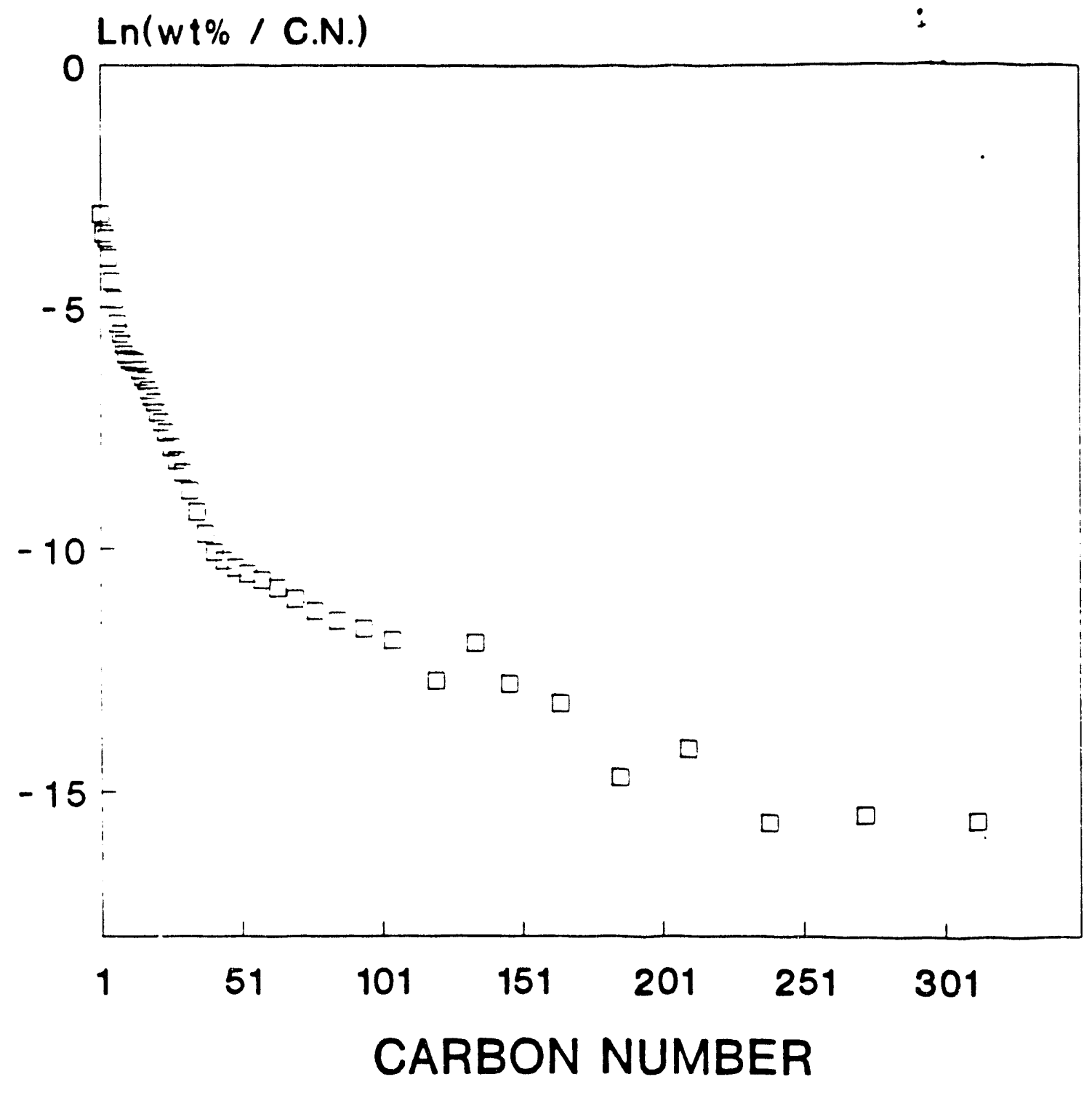

- EXPERIMENTAL 


\section{NONLINEAR REGRESSION OF F-T PROD. DIST PLANT 700B, RUN 42, PERIOD $6{ }^{\circ}$}

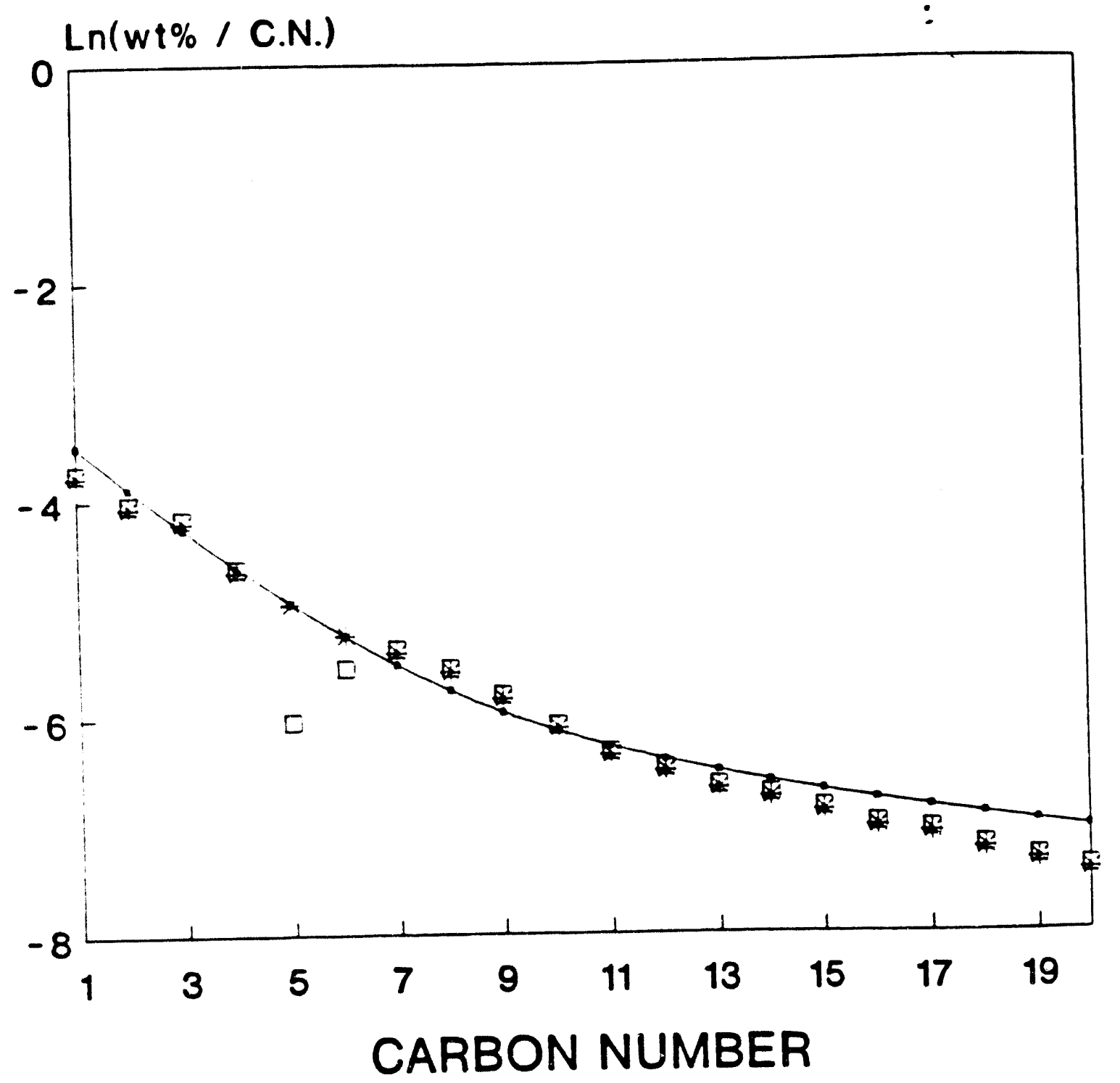

E Experimental $\rightarrow$ NLR Fitted * C5 C6 Crrctd

ALPHA 1=0.65; ALPHA 2=0.94; X1=7.2;

$\mathrm{PH}=0.672$

IRUN42IP6NLR.CHT 


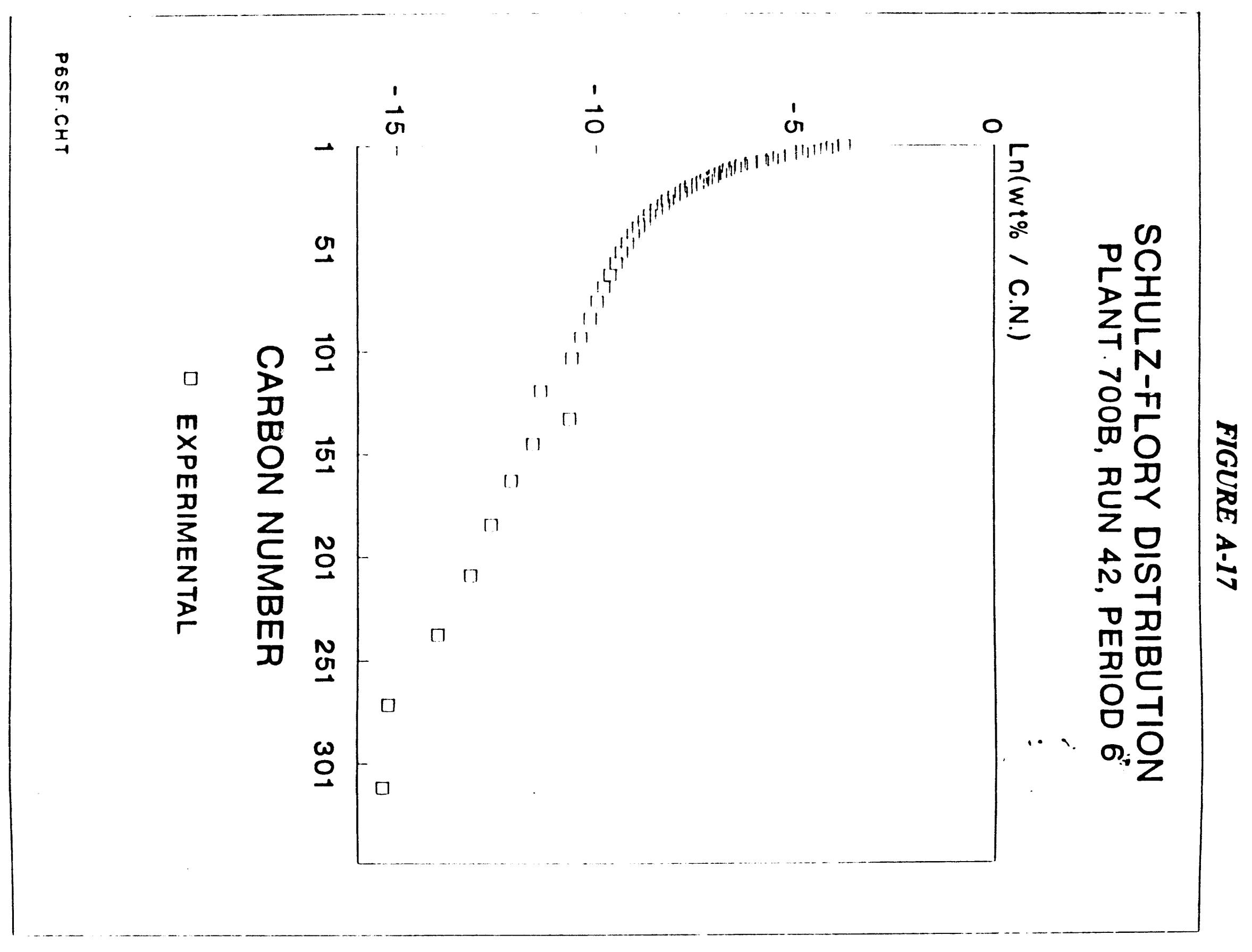




\section{NONLINEAR REGRESSION OF F-T PROD. DIS PLANT 700B, RUN 42, PERIOD \&.}

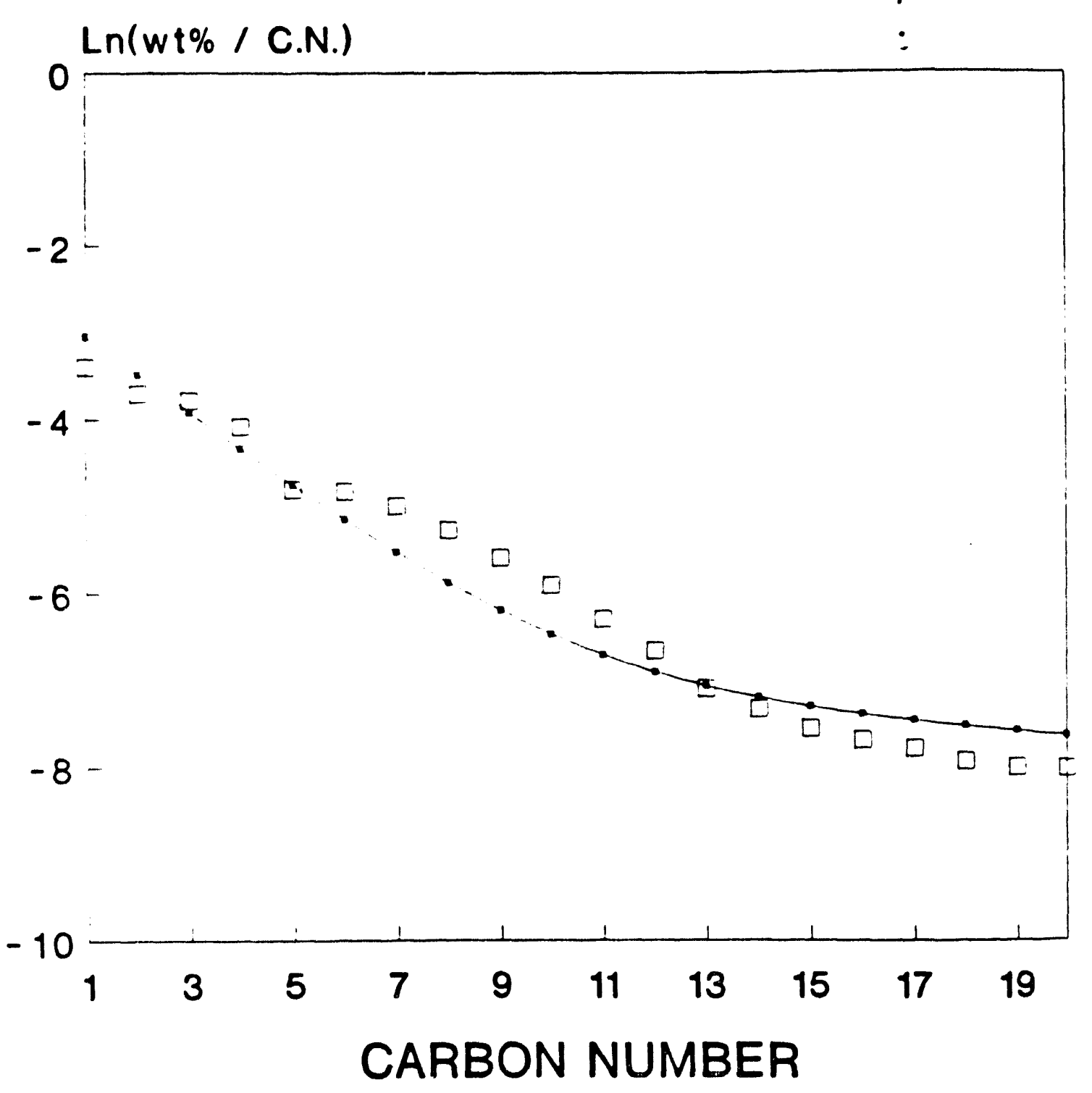

$\square$ Experimental $\rightarrow$ NLR Fitted

ALPHA $1=0.64 ;$ ALPHA $2=0.96 ; X 1=10.4 ;$

$P H I=2.572$

IRUN42 \PBNLR.CHT 


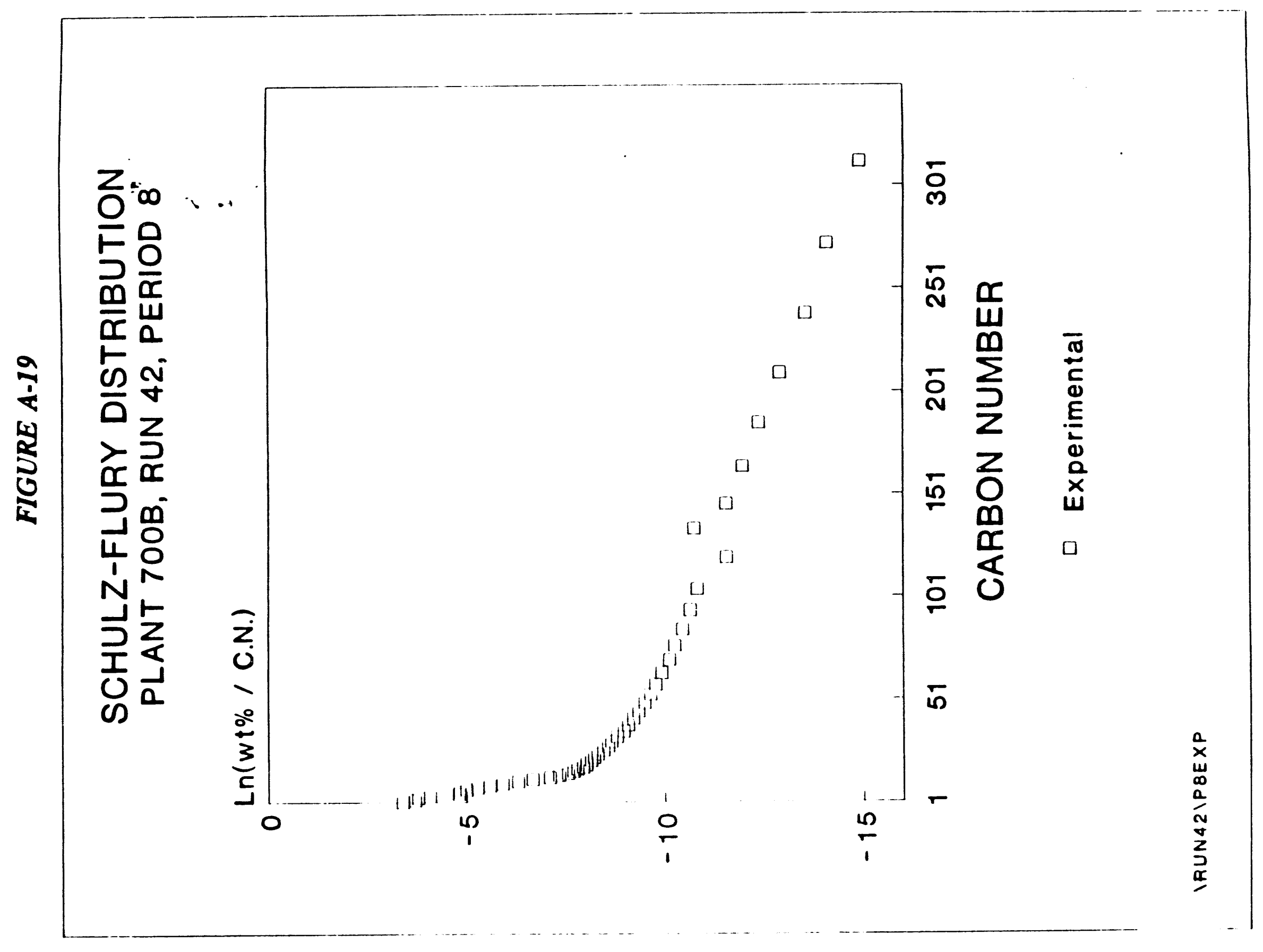




\section{FIGURE A-20 \\ HC PRODUCT DISTRIBUTION PLANT 700B RUN 42}

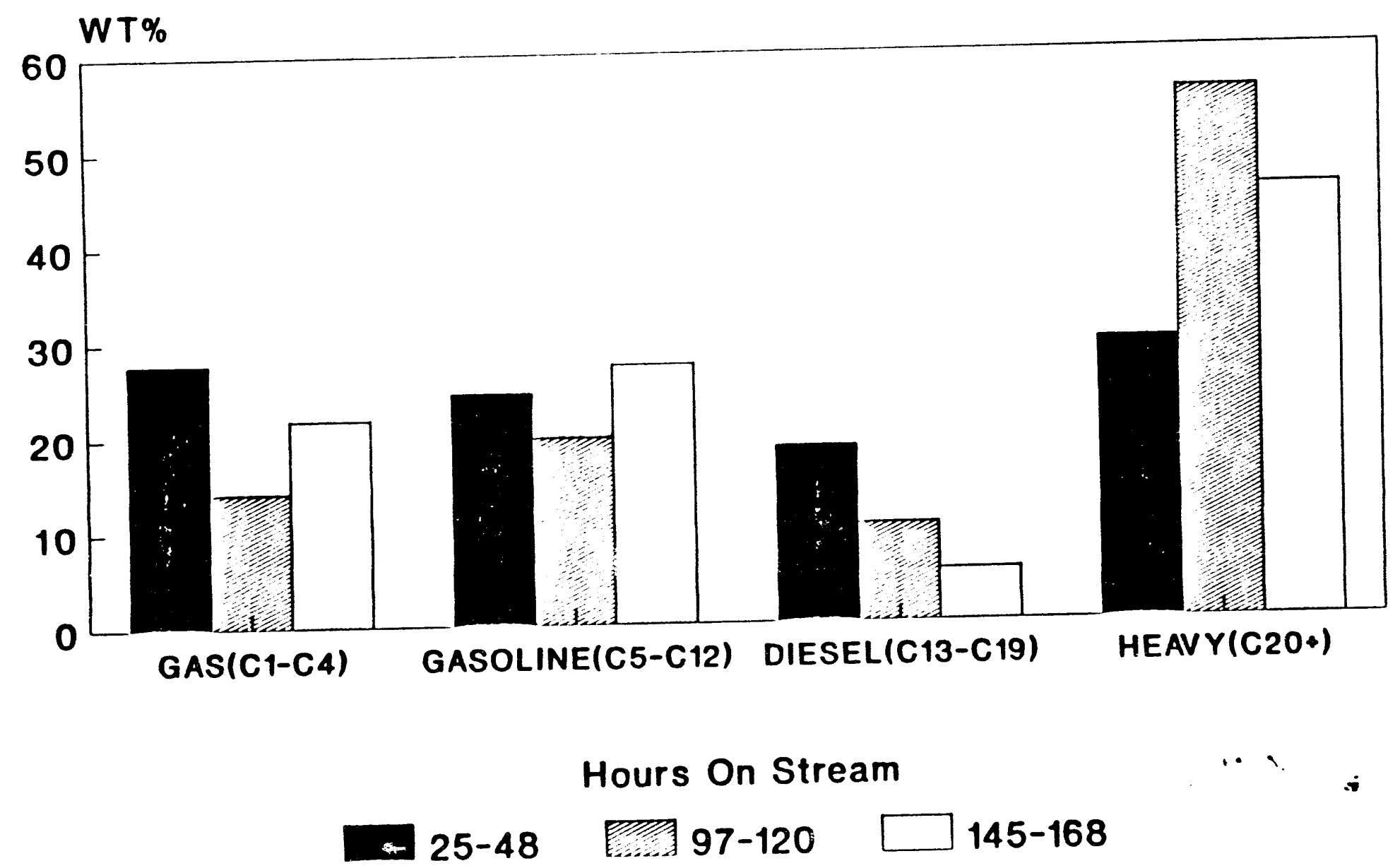

IRUN42IGRPDIS.CHT 


\section{FIGURE A-21}

\section{POTASSIUM LAURATE ADDTION TO SLURRY}

PL 700B RUN $43 \quad \mathrm{H}_{2}: \mathrm{CO}$ food $=0.7,1100 \mathrm{cpm}$

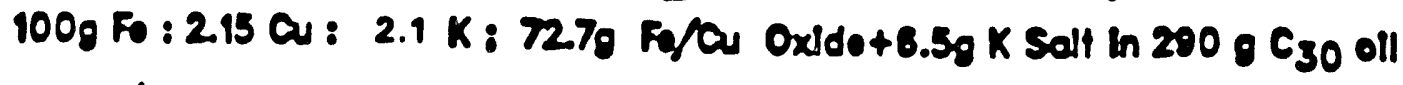
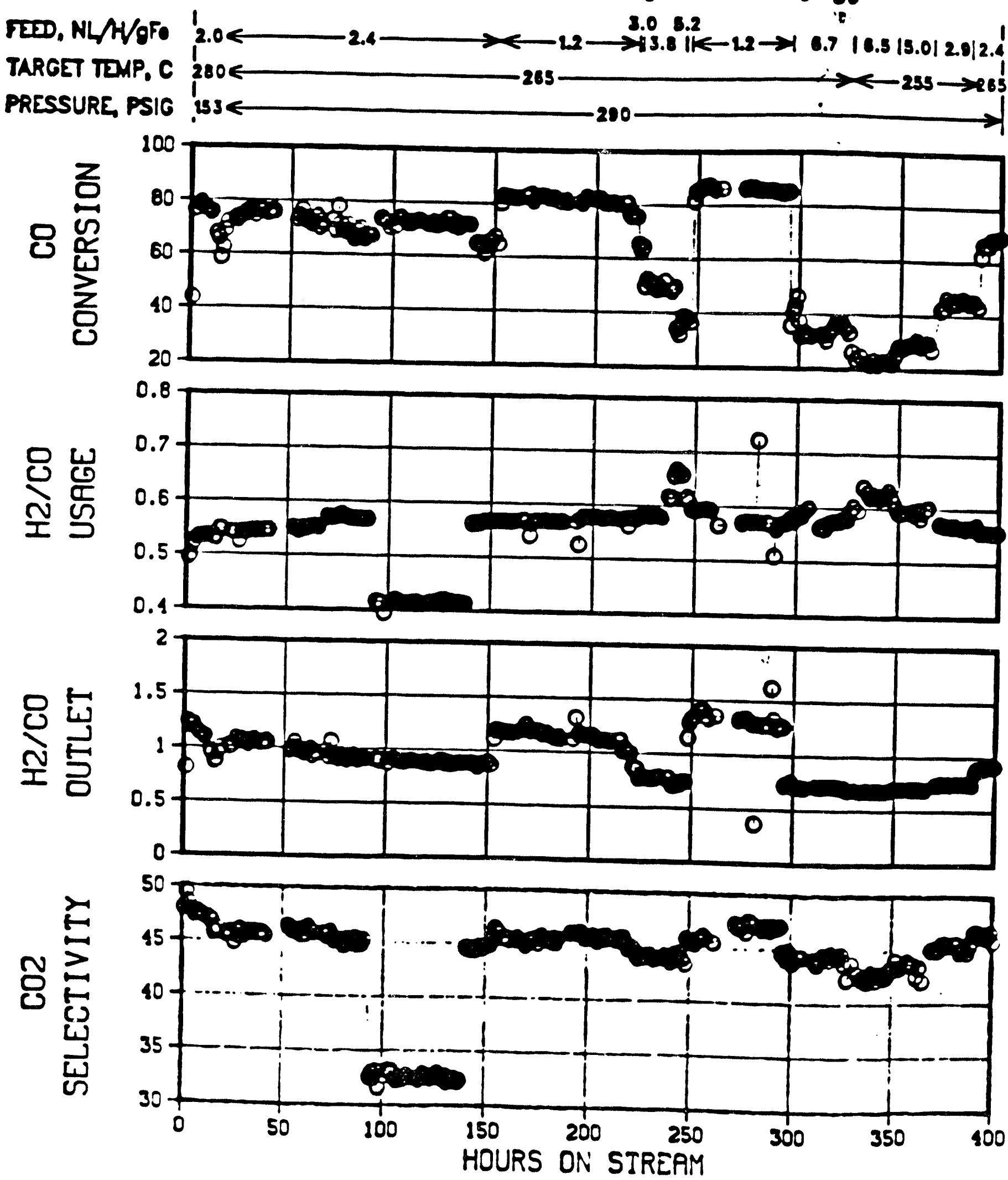
FIGURE A-22

POTASSIUM LAURATE ADDITON TO SLURRY

PU 700B RUN $43 \quad \mathrm{H}_{2}: \mathrm{CO}$ food $=0.7,1100 \mathrm{mpm}$

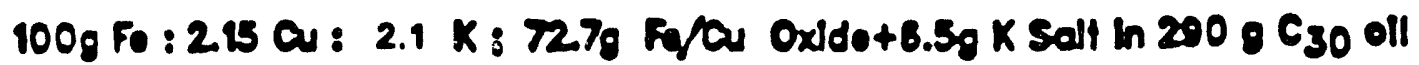
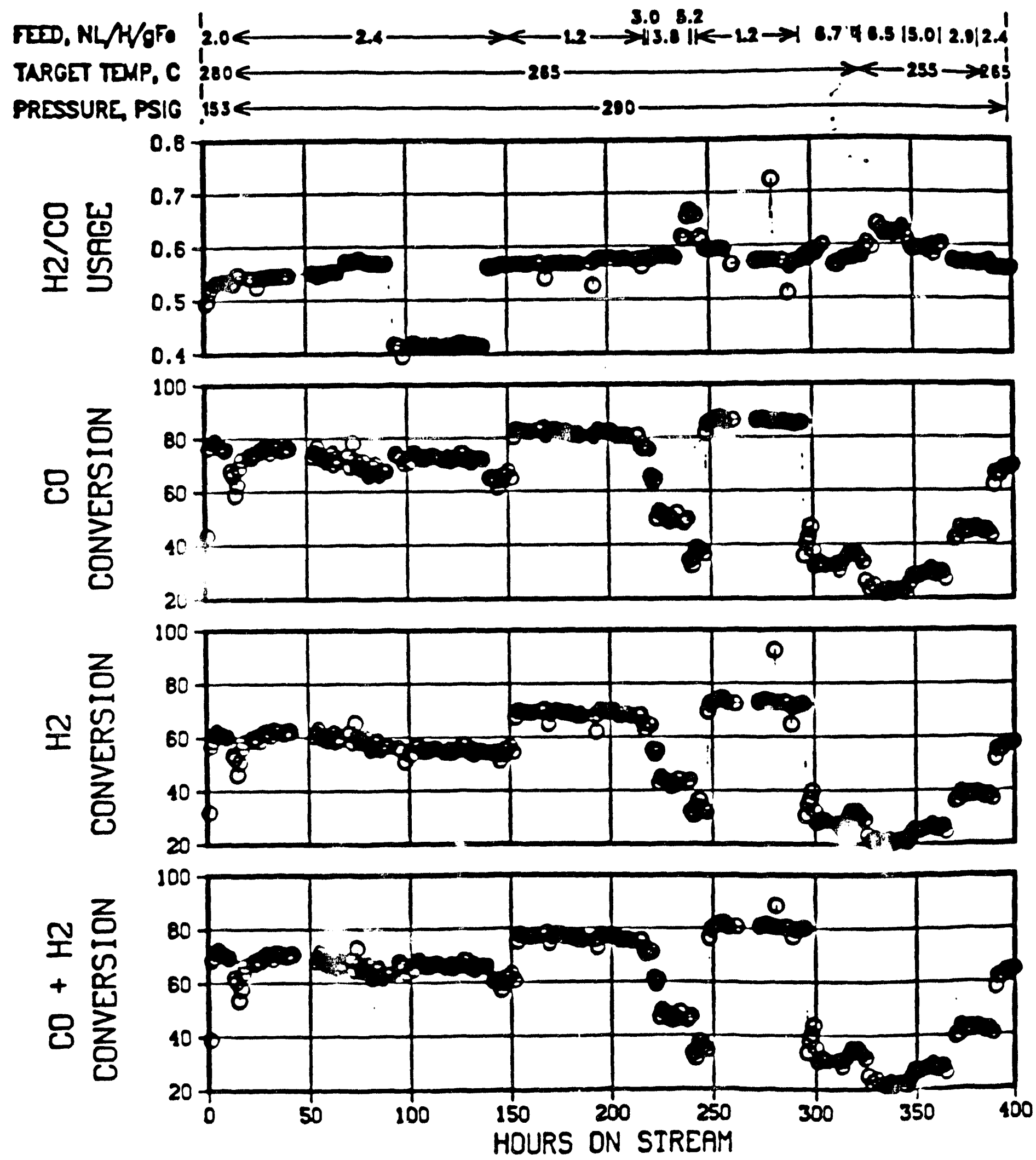


\section{POTASSIUM LAURATE ADDTION TO SLURRY PU 700B RUN $43 \quad \mathrm{H}_{2}: C 0$ foed $=0.7,1100 \mathrm{rpm}$}

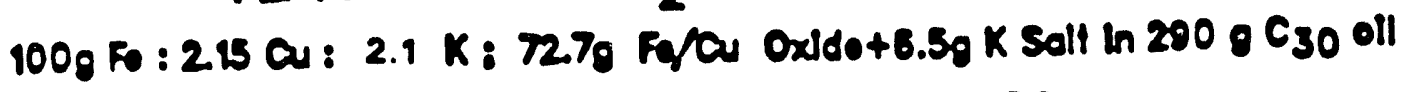

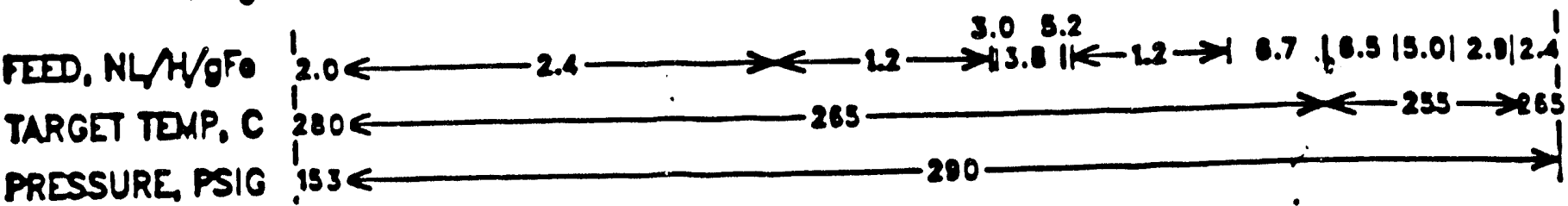
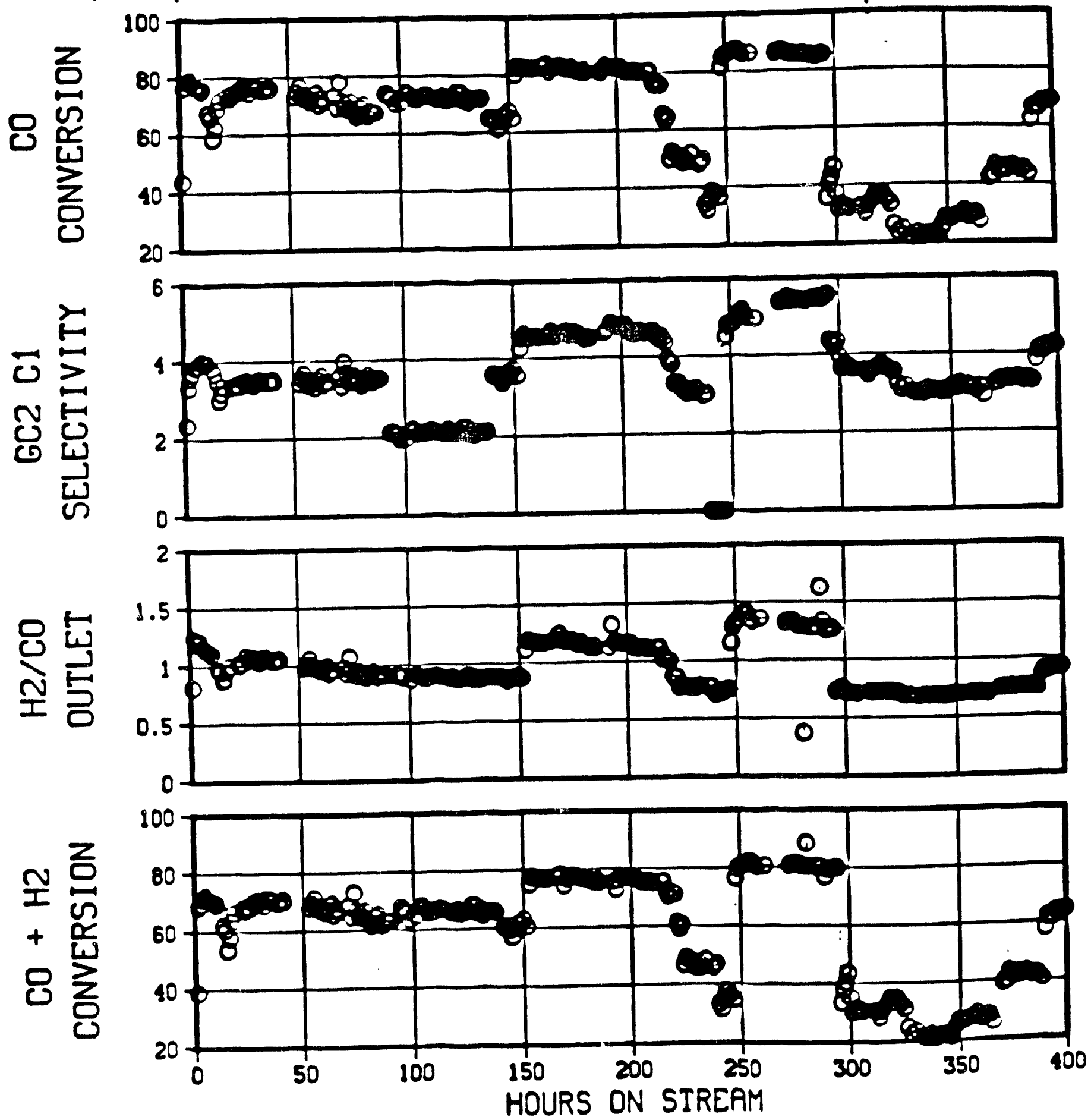
FIGURE A-24

POTASSIUM LAURATE ADDITON TO SLURRY

PL 700B RUN $43 \quad \mathrm{H}_{2}: \mathrm{CO}$ food $=0.7,1100 \mathrm{rpF}$

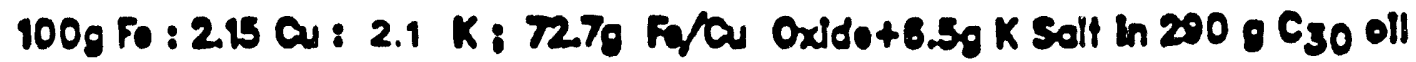
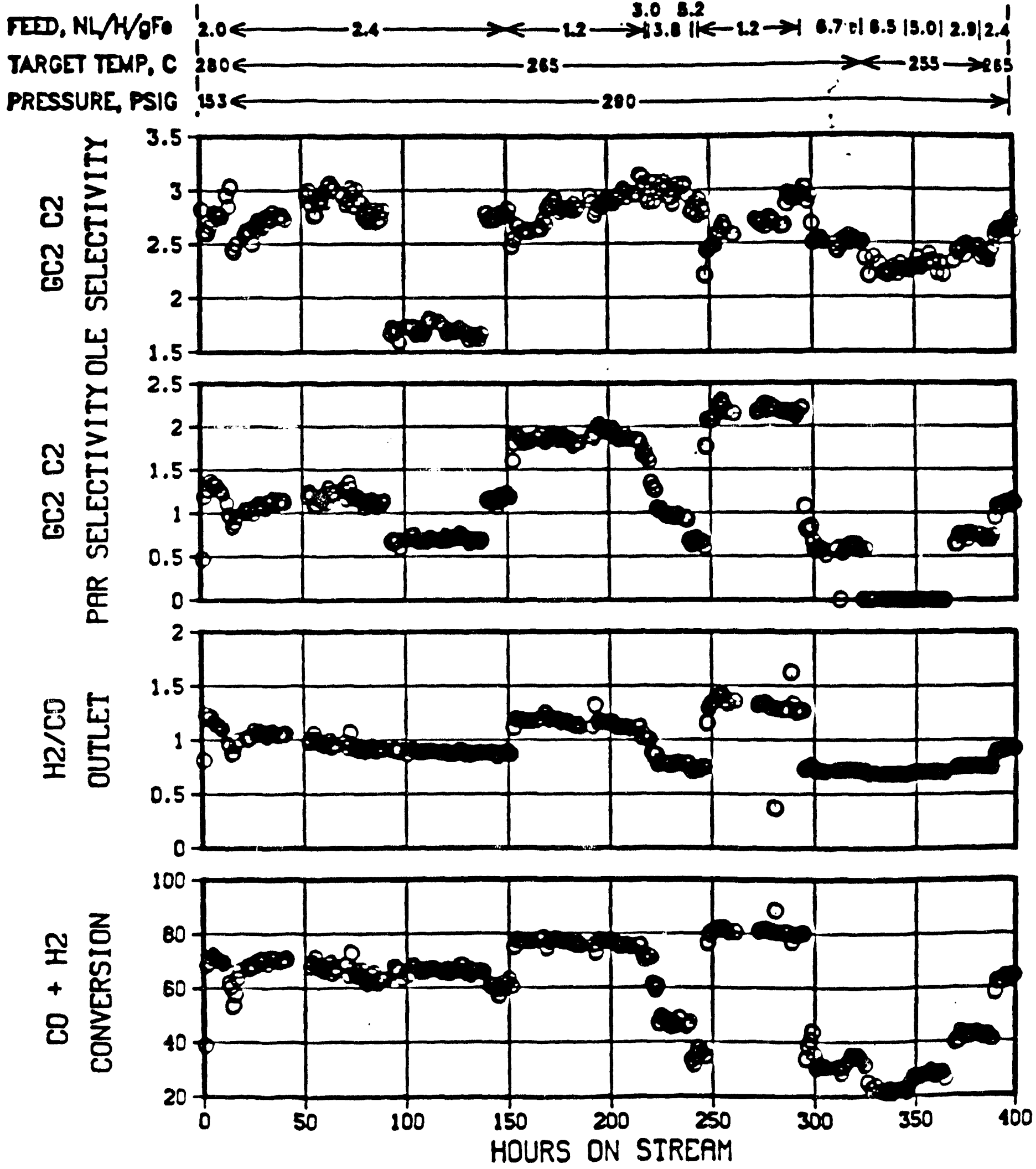
POTASSIUM LAURATE ADDITION TO SLURRY

PU 700B RUN $43 \quad \mathrm{H}_{2}: C 0$ foed $=0.7,1100 \mathrm{rpm}$

$100 \mathrm{~g}$ Fo : 2.25 C: : $2.1 \mathrm{~K}: 72.70 \mathrm{~F} / \mathrm{Cu}$ Oxido+6.50 K Solt in 290 \& $\mathrm{C}_{30}$ oll
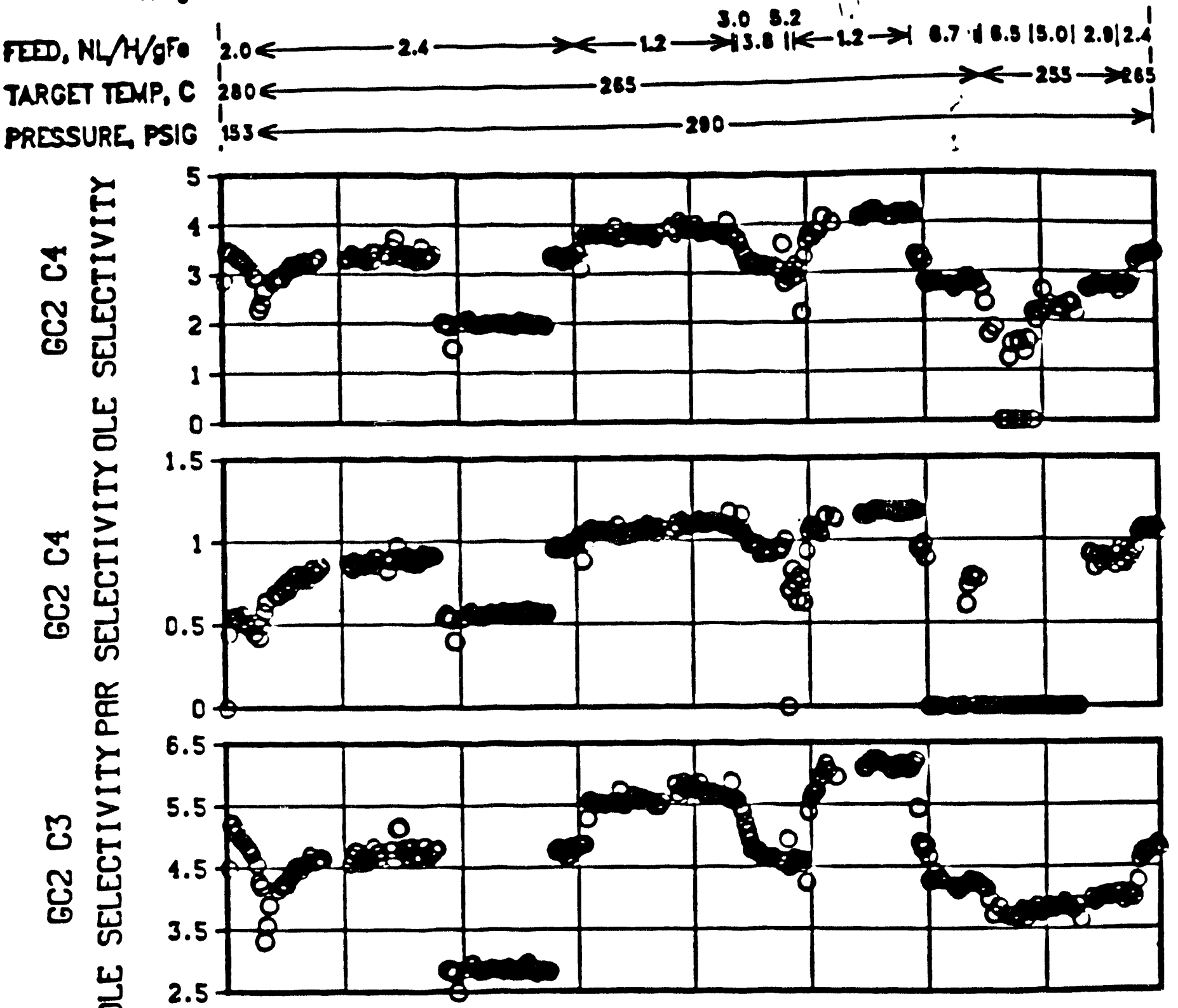

ש

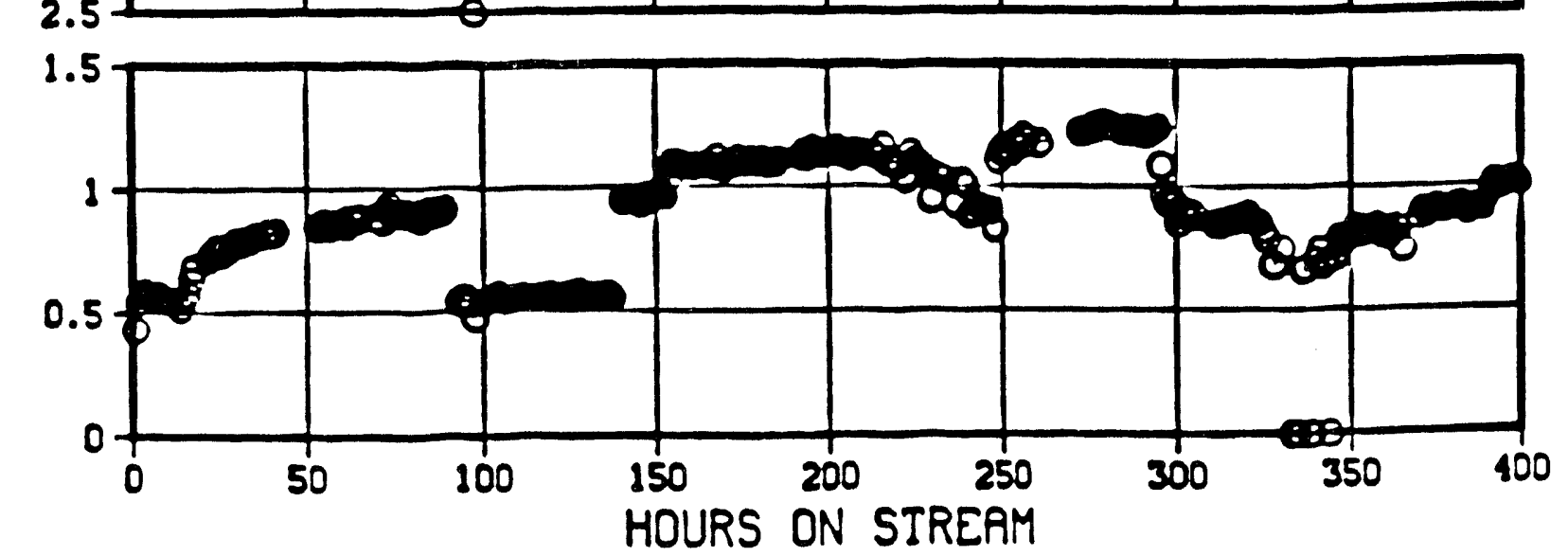


FIGURE A-26

POTASSIUM LAURATE ADDTION TO SLURRY

PIJ 700B RUN $43 \quad \mathrm{H}_{2}: C 0$ food $=0.7,1100 \mathrm{rpm}$

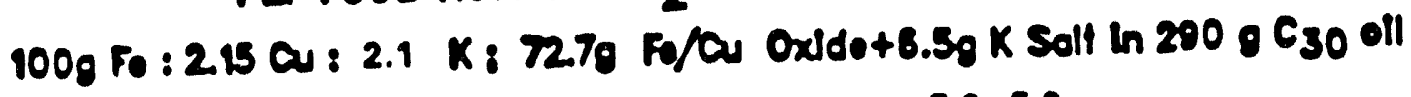

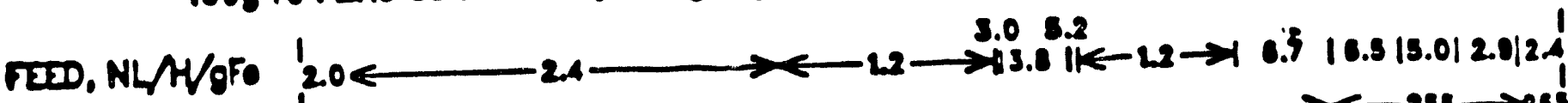

TARGET TEMP, C $280 \leftarrow$

PRESSURE, PSIC $253 \leftarrow$ 263

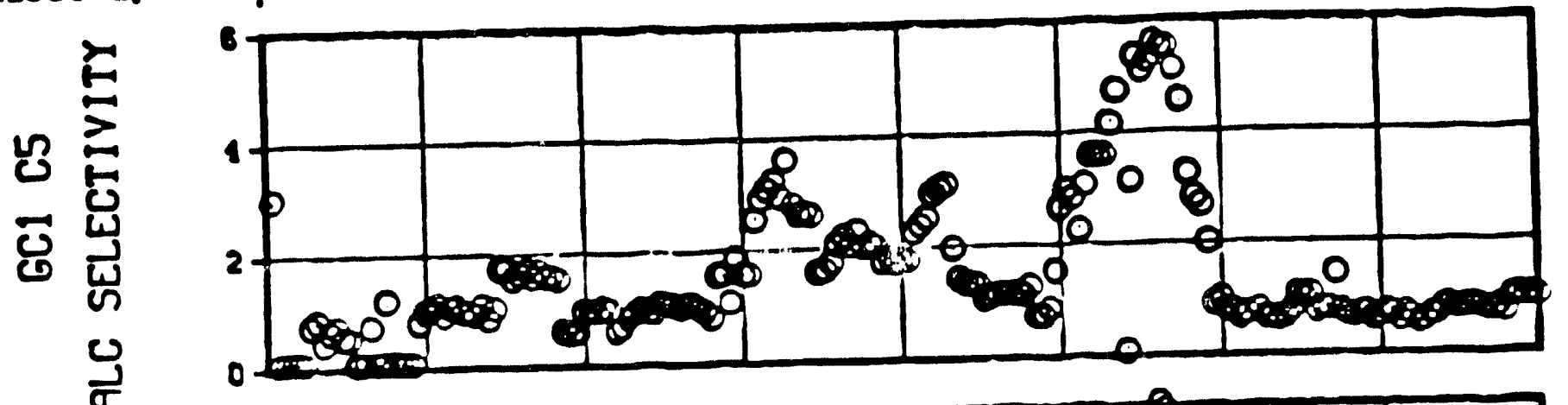

ठ

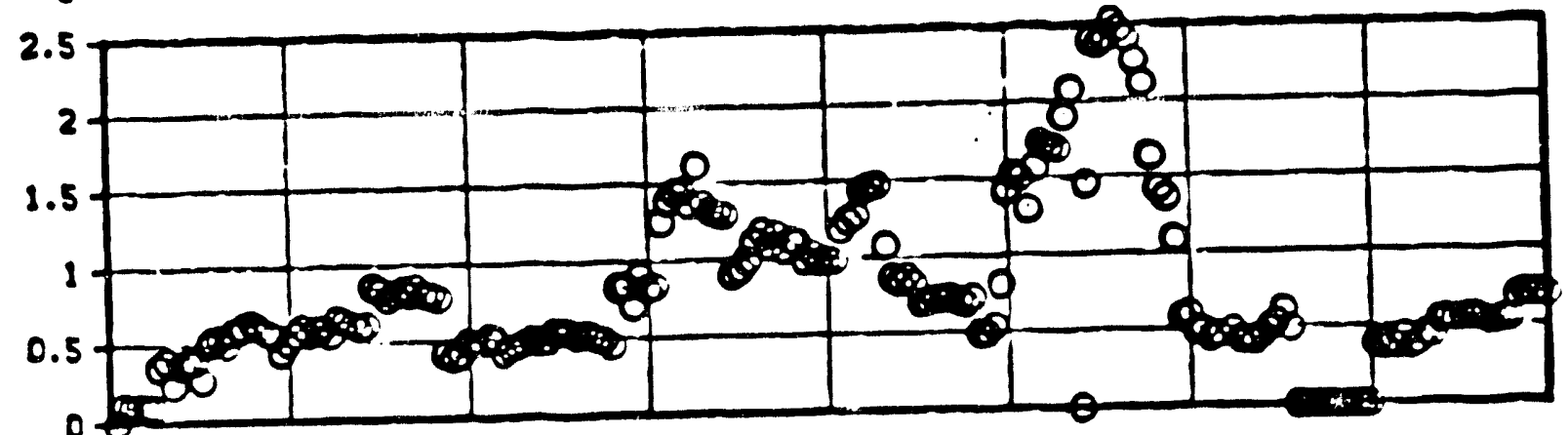

崩

ڤ

ن

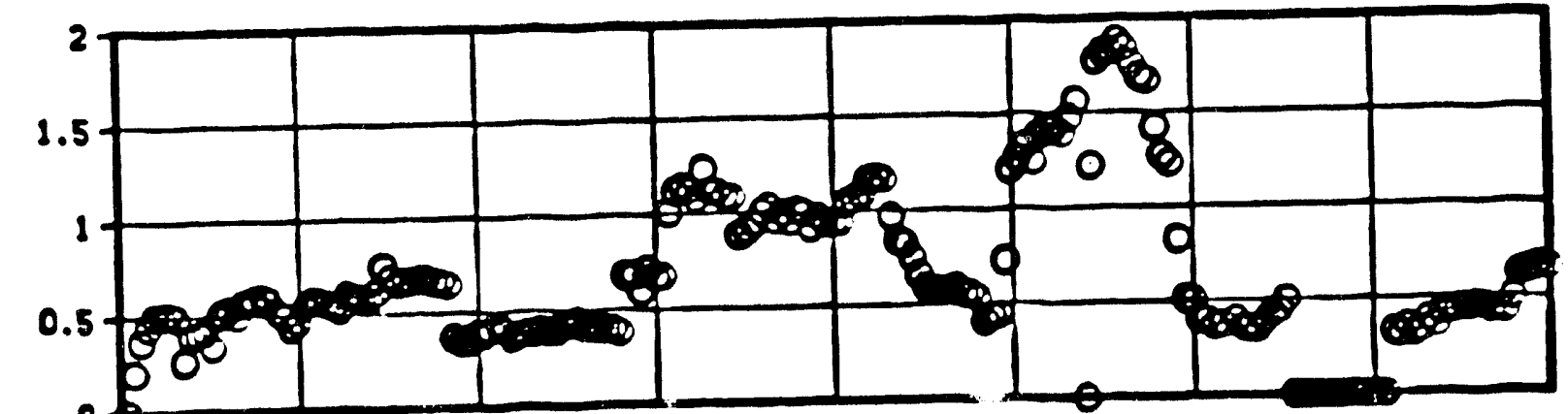

0

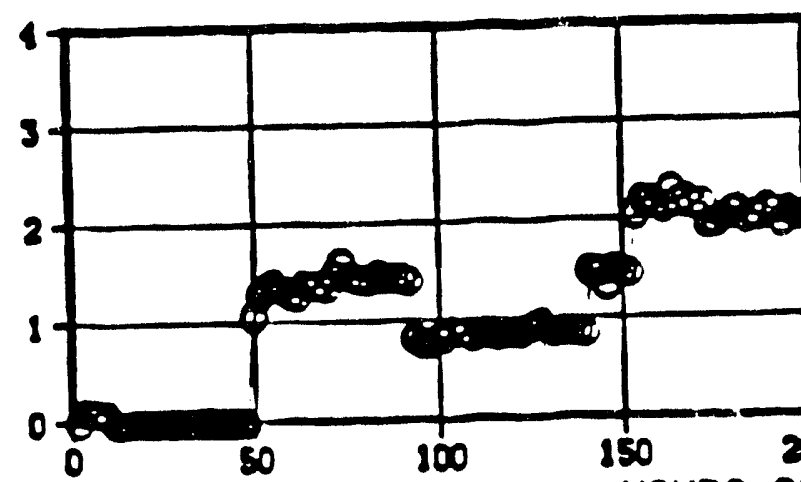

HOURS ON STREAM

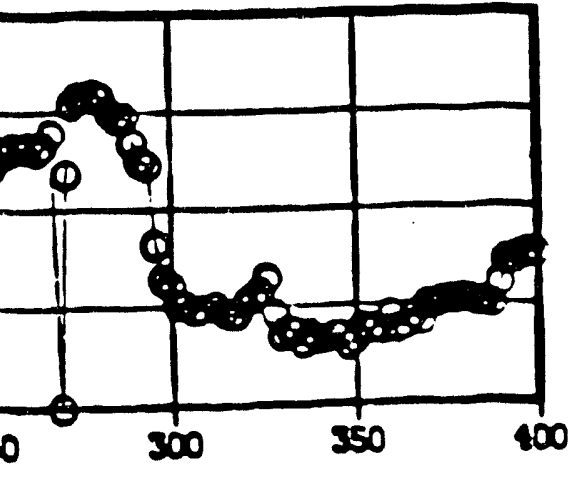




\section{FIGURE A-27}

P.ANT 70IE, RULK 4?, PERIDJ a ICTAL PRODJETS

\begin{tabular}{|c|c|c|c|c|c|c|c|c|c|c|}
\hline C 1 & $\begin{array}{l}\text { waX } \\
\text { wtz }\end{array}$ & $\begin{array}{l}\text { MAY } \\
\text { GraE }\end{array}$ & $\begin{array}{l}\text { ICE AD } \\
\text { GraA }\end{array}$ & $\begin{array}{r}\text { ICE HC } \\
\text { Gras }\end{array}$ & $\begin{array}{r}\text { CAYO AD } \\
\text { Grae }\end{array}$ & $\begin{array}{c}\text { CYRO HC } \\
\text { Gras }\end{array}$ & $\begin{array}{l}\text { GAS } \\
\text { Grat }\end{array}$ & $\begin{array}{c}\text { sin } \\
\text { Grae }\end{array}$ & กา & $\ln (N T Z / C N)$ \\
\hline Potal & & 167.64 & 0 & 63.99 & 0 & 21.98 & 43.1 & 296.71 & & \\
\hline 1 & 0 & 0.000 & 0 & 0 & 0 & 0 & 9.79 & 9.79 & 3.30 & -3.111 \\
\hline 2 & 0 & 0.000 & 0 & 3.71 & 0 & 1.07 & 10.08 & 14.86 & 3.01 & -3.687 \\
\hline 3 & 0 & 0.000 & 0 & 4.36 & 0 & 8.04 & 13.29 & 18.69 & 6.30 & -3.863 \\
\hline 4 & 0 & 0.000 & 0 & 8.48 & 0 & 3.2 & 9.94 & 21.62 & 7.29 & -4.005 \\
\hline 3 & 0 & 0.000 & 0 & 7.25 & 0 & 5.80 & & 13.13 & 4.43 & -1.727 \\
\hline 6 & 0.2 & 0.335 & c & 7.82 & 0 & 9.43 & & 13.39 & 4.58 & -4.876 \\
\hline 7 & 1.02 & 1.710 & 0 & 8.73 & & 3.15 & & 13.39 & 4.38 & -3.029 \\
\hline $\boldsymbol{E}$ & $1.9 E$ & 2.649 & & 7.82 & & 1.3 & & 11.77 & 3.97 & -9.307 \\
\hline 9 & 2.39 & 3.940 & & 6.01 & & 0.416 & & 10.37 & 3.49 & -5.351 \\
\hline 10 & 3.18 & 3.230 & & 4.04 & & 0.208 & & 9.48 & 3.19 & -5.746 \\
\hline$d 1$ & 3.84 & $6.43 ?$ & & 2.47 & & 0.182 & & 9.09 & 3.06 & -9.864 \\
\hline 12 & 1.29 & 7.192 & & 1.69 & & 0.104 & & 0.95 & 3.01 & -5.986 \\
\hline 13 & 1.11 & $7.39:$ & & 0.824 & & 0.026 & & 8.24 & 2.78 & -6.148 \\
\hline 14 & 1.53 & 7.594 & & 0.499 & & 0 & & 8.09 & 2.73 & -6.241 \\
\hline 19 & 4. (1) & $6.85 t$ & & 0.247 & & 0 & & 7.10 & 2.39 & -6.440 \\
\hline$d t$ & 3.68 & 6.169 & & $0.0 E 2$ & & 0 & & 6.25 & 2.11 & -6.633 \\
\hline 17 & 3.9 & 9.867 & & 0 & & & & 5.87 & 1.98 & -6.757 \\
\hline 18 & 2.98 & $4.99 \mathrm{e}$ & & 0 & & & & 3.06 & 1.68 & -6.975 \\
\hline 19 & i.t! & 1.442 & & 0 & & & & 4.44 & 1.50 & -7.146 \\
\hline$\because$ & $2.9 ?$ & $3.97 ?$ & & & & & & 3.97 & 1.34 & -7.309 \\
\hline $2 ! .0 !$ & $1.9:$ & $3.2: 2$ & & & & & & 3.20 & 1.08 & -7.576 \\
\hline 32.6 & $1.6:$ & $2.76 ?$ & & & & & & 2.76 & 0.94 & -7.787 \\
\hline 24.25 & $1.3 \mathrm{i}$ & 2.915 & & & & & & 2.5! & 0.85 & -7.939 \\
\hline $2 t .05$ & 1.29 & 2.163 & & & & & & 2.16 & 0.73 & $-8.18 !$ \\
\hline $36.0 ?$ & $1.1:$ & $1.87 i$ & & & & & & 1.86 & 0.63 & -8.397 \\
\hline 30.2 & 1.01 & 1.743 & & & & & & 1.74 & 0.59 & -8.545 \\
\hline 39.98 & 0.85 & 1.492 & & & & & & 1.19 & 0.30 & -8.775 \\
\hline 39.2 & 0.78 & $1.3 \cap 8$ & & & & & & 1.31 & 0.44 & -8.980 \\
\hline $36 . !$ & 0.73 & 1.224 & & & & & & 1.22 & 0.41 & -9.131 \\
\hline 11.3 & 0.64 & 1.073 & & & & & & 1.07 & 0.36 & -9.343 \\
\hline 41.85 & 0.39 & 0.969 & & & & & & 0.99 & 0.33 & -9.307 \\
\hline AE.B & 0.94 & 0.995 & & & & & & 0.91 & 0.31 & -7.680 \\
\hline 59.28 & 0.19 & 0.828 & & & & & & 0.82 & 0.28 & -9.864 \\
\hline 56.2 & c.46 & 0.771 & & & & & & 0.77 & 0.26 & -10.017 \\
\hline 63.79 & 0.42 & 0.704 & & & & & & 0.70 & 0.24 & -10.199 \\
\hline 70.03 & 0.97 & 0.620 & & & & & & 0.62 & 0.21 & -10.420 \\
\hline 77.15 & 0.32 & 0.336 & & & & & & 0.31 & 0.18 & -10.661 \\
\hline $85 . .23$ & 0.27 & 0.453 & & & & & & 0.15 & 0.19 & -10.930 \\
\hline 91.25 & 0.23 & $0.38:$ & & & & & & 0.39 & 0.13 & -11.192 \\
\hline di1.s:s & 0.19 & 0.319 & & & & & & 0.32 & 0.11 & $-11.48 i$ \\
\hline
\end{tabular}




\section{FIGURE A-27 (CONT)}

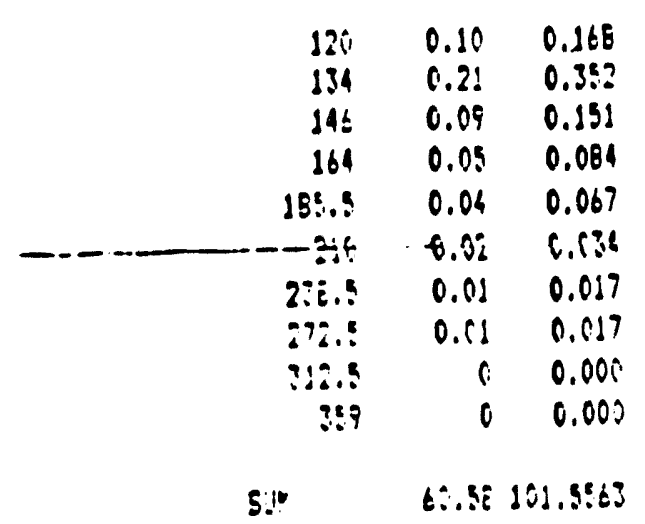

$120 \quad 0.10 \quad 0.168$

$134 \quad 0.2 ! \quad 0.35 ?$

d4t 0.090 .000 .084

$\begin{array}{lll}164.0 & 0.04 & 0.067\end{array}$

$-24 \quad 0.02 \quad 0.136$

$\begin{array}{lll}230.5 & 0.11 & 0.017\end{array}$

tast do1.s?63

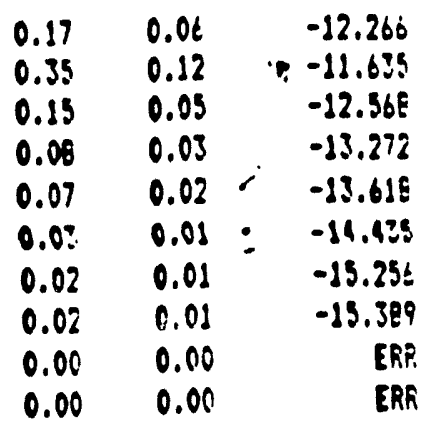

$\begin{array}{lll}0 & 63.992 & 0 \\ 22.006\end{array}$

43.1230 .634377 .73728 
FIGURE A-28 (CONT)

\begin{tabular}{|c|c|c|}
\hline $\begin{array}{l}\text { 8s. is } \\
88.0^{2}\end{array}$ & $\begin{array}{l}0.6: \\
\text { C.36 }\end{array}$ & $\begin{array}{l}0 . i j \\
0 . i k\end{array}$ \\
\hline : & $0.8=$ & c.::A \\
\hline :Z: & D..? & C.064 \\
\hline 134 & 0.34 & 0.146 \\
\hline 146 & 0.19 & 0.064 \\
\hline 164 & 0.10 & $0: 043$ \\
\hline 108.3 & 0.07 & 0.030 \\
\hline 210 & 0.05 & 0.022 \\
\hline 238.5 & 0.03 & 0.013 \\
\hline 272.5 & 0.02 & 0.007 \\
\hline 312.5 & 0.01 & 0.004 \\
\hline 357 & 0 & 0.000 \\
\hline
\end{tabular}

$\begin{array}{lll}0.11 & 0.12 & -11.170 \\ 0.15 & 0.10 & -11.435 \\ 0.12 & 0.09 & -11.697 \\ 0.06 & 0.01 & -12.494 \\ 0.15 & 0.10 & -11.716 \\ 0.01 & 0.01 ; & -12.690 \\ 0.01 & 0.03 ; & -13.212 \\ 0.03 & 0.02: & -13.692 \\ 0.02 & 0.02 & -11.113 \\ 0.01 & 0.01 & -11.791 \\ 0.01 & 0.01 & -15.529 \\ 0.00 & 0.00 & -16.160 \\ 0.00 & 0.00 & 2218\end{array}$

$43.63218 .4448^{\circ}$

1.9914 .999

- 20.972

30.13186 .556811 .52878 
FIGURE A-29

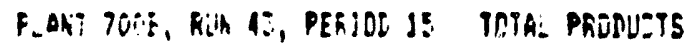

\begin{tabular}{|c|c|c|c|c|c|c|c|c|c|c|}
\hline 61 & $\begin{array}{l}n=1 \\
n+1\end{array}$ & $\begin{array}{l}\text { Wint } \\
\text { Gras }\end{array}$ & $\begin{array}{l}\text { ICE AO } \\
\text { Grat }\end{array}$ & $\begin{array}{r}\text { ICE HE } \\
\text { Gras }\end{array}$ & $\begin{array}{l}\text { CRYO AD } \\
\text { Grae }\end{array}$ & $\begin{array}{l}\text { CrRs HE } \\
\text { Grae }\end{array}$ & $\begin{array}{r}\text { 6A5 } \\
\text { 6ra1 }\end{array}$ & $\begin{array}{l}\text { SUR } \\
\text { Gran }\end{array}$ & rit & $\ln \left(x+y / \operatorname{sn}_{i}\right.$ \\
\hline Yois: & & 194.5 & 1.093 & 62.99 & 2.291 & $1 E$ & 53.93 & 292.85 & & 't \\
\hline 1 & 0 & 0.000 & 0 & 0 & 0 & 0 & 16.09 & 16.09 & 3.50 & -2.901 \\
\hline 2 & 0 & 0.000 & 0.719 & 1.22 & 1.5 & 0.781 & 10.84 & 15.06 & 5.14 & -3.661 \\
\hline 3 & 0 & 0.000 & 0.213 & 2.69 & 0.313 & 0.809 & 15.06 & 19.28 & 6.96 & -3.819 \\
\hline 4 & 0 & 0.000 & 0.114 & 4.73 & 0.196 & 1.47 & 0.56 & 15.07 & 5.15 & -1.359 \\
\hline$?$ & 0 & 0.010 & 0.047 & 3.54 & 0.082 & 3.03 & & 8.10 & 2.97 & -5.126 \\
\hline 6 & 0.92 & 0.494 & 0 & 3.95 & 0 & 3.62 & & 10.06 & 3.44 & -9.162 \\
\hline 7 & 0.4 & 0.618 & 0 & 7.33 & & 2.81 & & 10.76 & 3.67 & -9.250 \\
\hline 8 & 0.83 & $1.2 E 2$ & & 8.15 & & 2.44 & & 11.87 & 4.05 & -5.285 \\
\hline 9 & 1.2 & 1.834 & & 7.66 & & 1.58 & & 11.09 & 3.79 & -5.470 \\
\hline 10 & $1.5 \hat{E}$ & 2.441 & & 6.11 & & 0.85: & & 9.40 & 3.21 & -3.741 \\
\hline 11 & 1.78 & 2.750 & & 4.32 & & 0.378 & & 7.45 & 2.54 & -6.069 \\
\hline 12 & $1.9 i$ & $2.9 t \dot{8}$ & & 3.5 & & $0.16 t$ & & 6.63 & 2.27 & -6.272 \\
\hline $1 ?$ & 2.05 & 3.167 & & 2.36 & & 0.071 & & 3.60 & 1.91 & -6.562 \\
\hline 16 & $\therefore \therefore:$ & 3.414 & & $! .47$ & & 0 & & 4.85 & $1.6 ?$ & $-6.73 ?$ \\
\hline $1:$ & i.d & $3.2 \div 4$ & & C.EFi & & 0 & & 4.14 & 1.41 & -6.967 \\
\hline it & $2 \therefore:$ & $\therefore . . \cap 8$ & & $0.5 ;$ & & & & 3.68 & 1.26 & -7.150 \\
\hline$!$ & 2.1 & 3.245 & & $0.32 t$ & & & & 3.57 & 1.22 & $-7.24:$ \\
\hline $1 \equiv$ & 1,54 & 2.99" & & C.1E? & & & & 3.16 & 1.05 & -7.419 \\
\hline$! 9$ & $\therefore \because ?$ & $\therefore 9 \hat{E}:$ & & & & & & 2.58 & 1.02 & -7.528 \\
\hline$i$ & 1.54 & $2.9 \div$ & & & & & & 3.00 & 1.62 & -7.978 \\
\hline$\because \therefore: \leq$ & $\therefore \ddot{E}:$ & 2.:: & & & & & & 8.76 & 0.93 & $-7.704^{-}$ \\
\hline$\because:$. & $1.9:$ & $\therefore \%$ & & & & & & $2.6 ?$ & $0.9 !$ & $.-7.8: 4$ \\
\hline$\therefore: \therefore 8$ & $1.6^{6}$ & ¿..:: & & & & & & 8.6! & 0.87 & $0-9,9 i E$ \\
\hline${ }_{t}, \therefore ?$ & $\therefore .85$ & 2.:?! & & & & & & 2.39 & 0.22 & -8.0156 \\
\hline 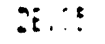 & $1.8:$ & 2.19: & & & & & & 2.15 & 0.75 & $-E .23 E$ \\
\hline$\therefore:$ & $\therefore .:=$ & $\therefore: 4 \vdots$ & & & & & & 2.15 & $6.7 \vdots$ & $-8 . .2 ?$ \\
\hline$\because: . ! !$ & $\therefore .26$ & d. $5: 8$ & & & & & & $1.5 \%$ & c.t? & $-\varepsilon .8 .1\}$ \\
\hline$\because \because 8$ & $1 .: 2$ & 1.:4: & & & & & & $\therefore .75$ & C.6? & $-E .6 E:$ \\
\hline$\therefore .:$ & 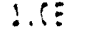 & 1.6:: & & & & & & 1.67 & 0.87 & $-i, 5 . i$ \\
\hline a: & 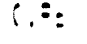 & $\therefore .4:$ & & & & & & $1.4 E$ & 0.51 & $-5.0 i=$ \\
\hline$\therefore:$ : : & 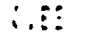 & $\therefore \therefore$ & & & • & & & $1 . j t$ & $0.4 \leqslant$ & $-9 .: 7 i$ \\
\hline $6: 8$ & $\therefore i$ & $\therefore \therefore$ & & & & & & 1.22 & 0.12 & $-5.35 \vdots$ \\
\hline :.:: & $6 \because \because$ & $\therefore:::$ & & & & & & 1.11 & $0.3 E$ & $-9.94:$ \\
\hline$\because::$ & $6.6^{2}$ & d.c.: 6 & & & & & & 1.06 & 0.84 & -9.73 \\
\hline ti:i! & $0.8:$ & C.Eंi & & & & & & 0.90 & 0.31 & -9.946 \\
\hline $7 . .25$ & c.:: & S.7EE & & & & & & 0.79 & 0.27 & -10.167 \\
\hline $79.2 !$ & $(.15$ & 0.455 & & & & & & 0.70 & 0.24 & -10.389 \\
\hline Es..15 & 6.35 & o.t: & & & & & & 0.60 & 0.21 & -10.631 \\
\hline 34.28 & 0.23 & c.s:: & & & & & & 0.51 & 0.17 & -80.899 \\
\hline $104.5:$ & 0.27 & 0.417 & & & & & & 0.12 & 0.14 & -11.203 \\
\hline$\because:$ & 0.24 & 0.216 & & & & & & 0.22 & 0.07 & -81.998 \\
\hline$!: 4$ & 0.31 & 0.479 & & & & & & 0.48 & 0.16 & -21.314 \\
\hline 146 & 0.14 & 0.216 & & & & & & 0.22 & 0.07 & -12.194 \\
\hline 164 & 0.69 & 0.139 & & & & & & 0.14 & 0.05 & $-12.75 ?$ \\
\hline $189 !$ & C.l.t & $0.09 i$ & & & & & & 0.05 & 0.03 & -13.281 \\
\hline 210 & 0.04 & 0.062 & & & & & & 0.06 & 0.02 & -83.810 \\
\hline
\end{tabular}


FIGURE A-29 (CONT)

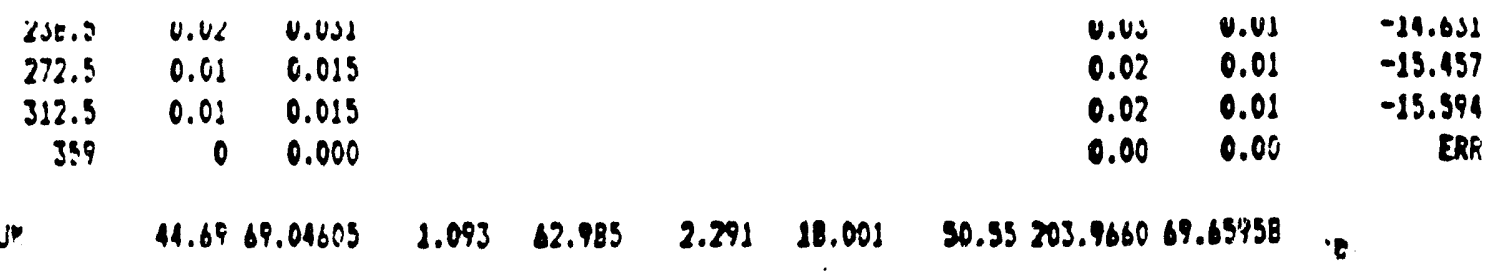




\section{NONLINEAR REGRESSION OF F-T PRODUCTS PLANT 700B, RUN 43, PERIOD 4 "}

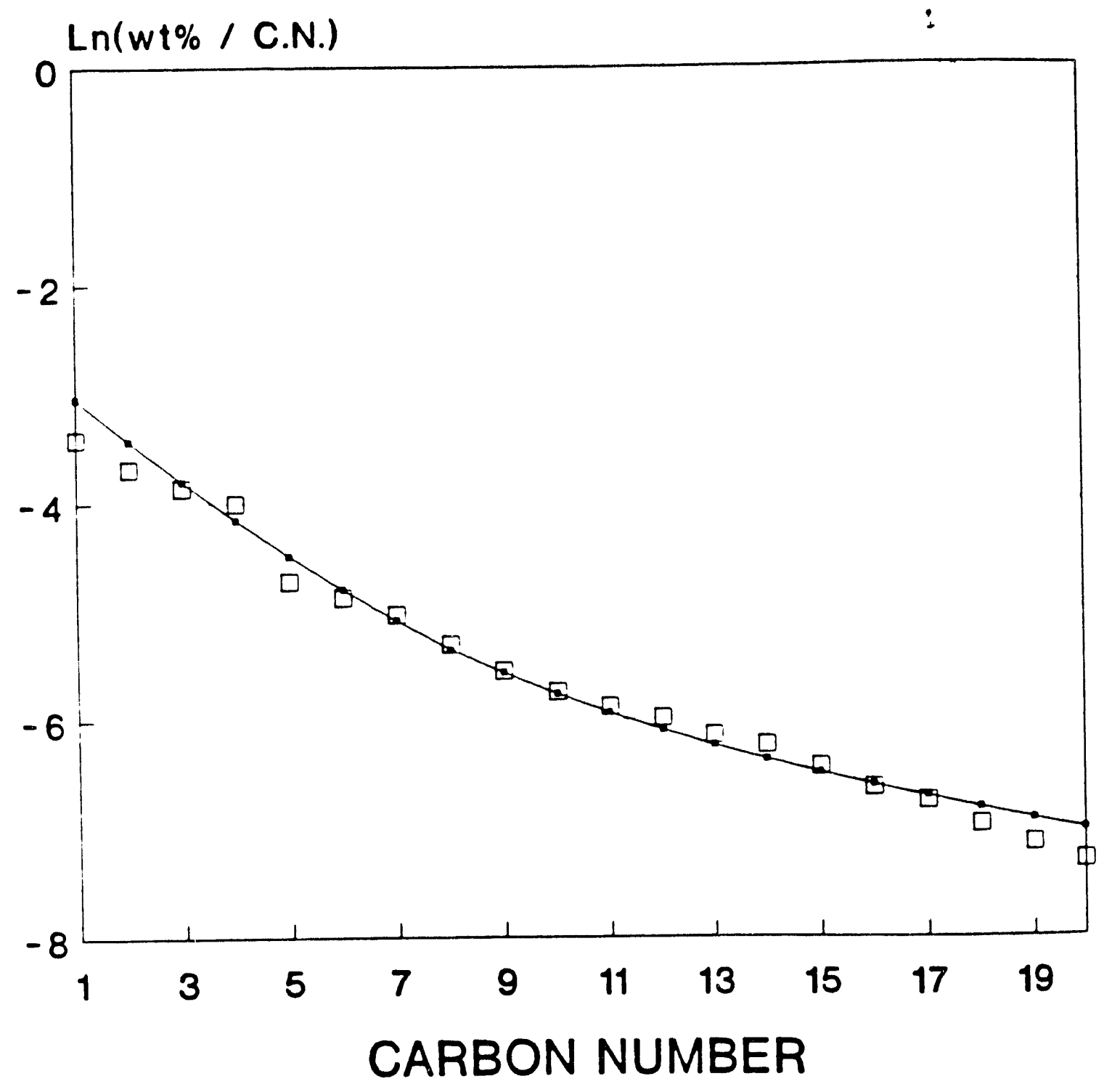

$\square$ Experimental $\rightarrow$ NLR Fitted

ALPHA 1=0.65; ALPHA 2-0.91; X1=7.2; 


\section{SCHULZ-FLORY DISTRIBUTION PLANT 700B, RUN 43, PERIOD 4}

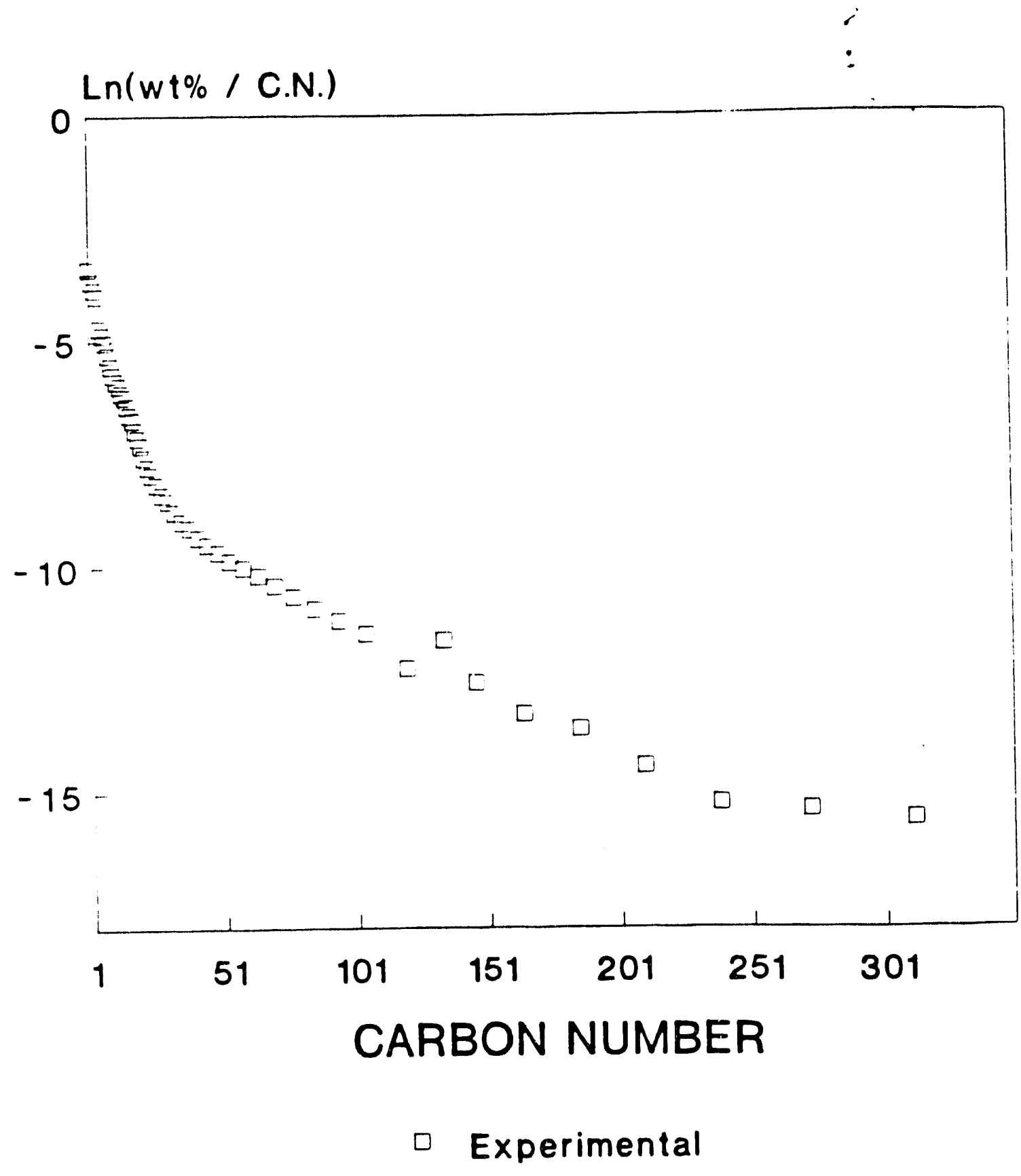




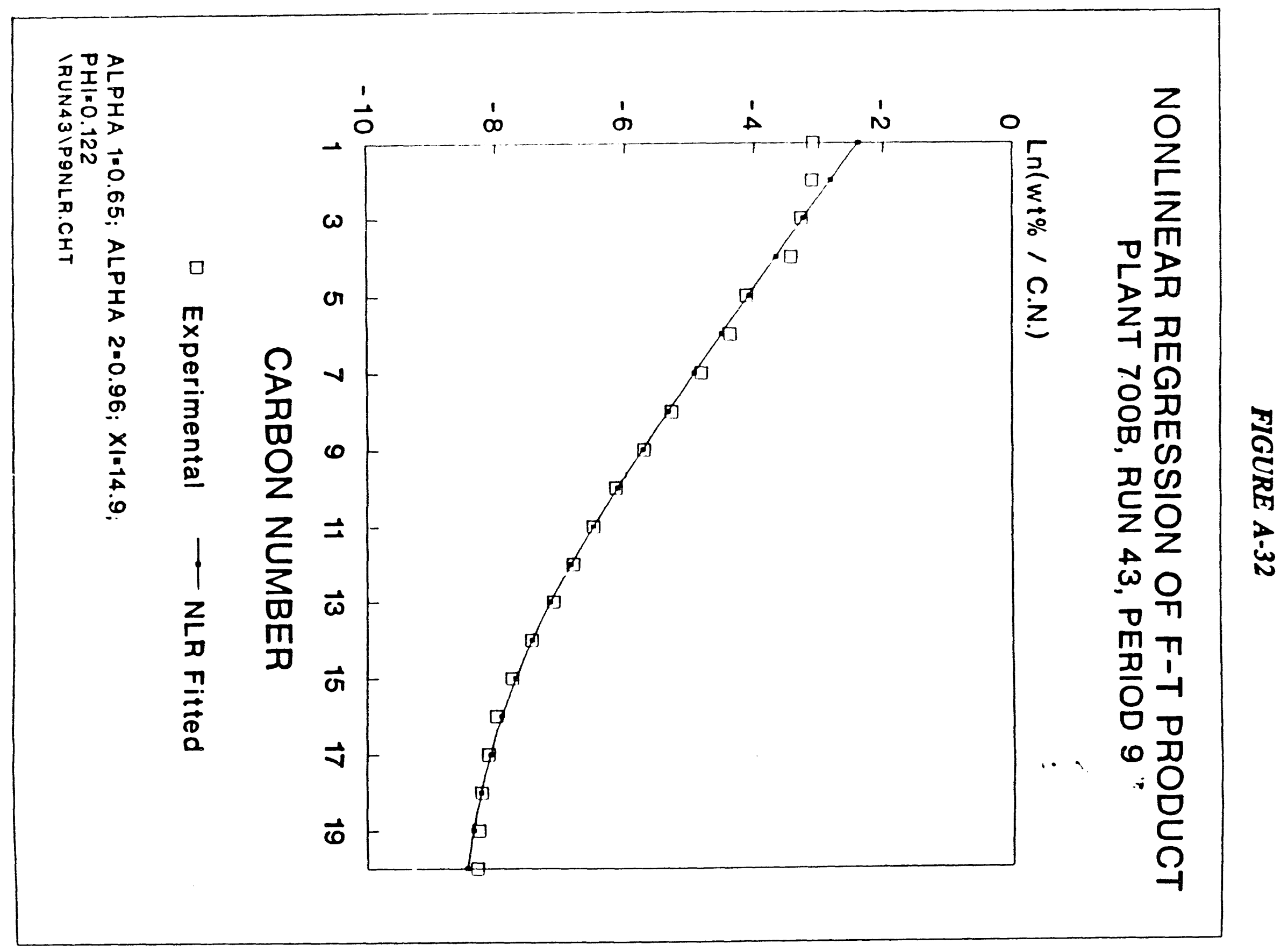




\section{SCHULZ-FLORY DISTRIBUTION PLANT 700B, RUN 43, PERIOD 9}

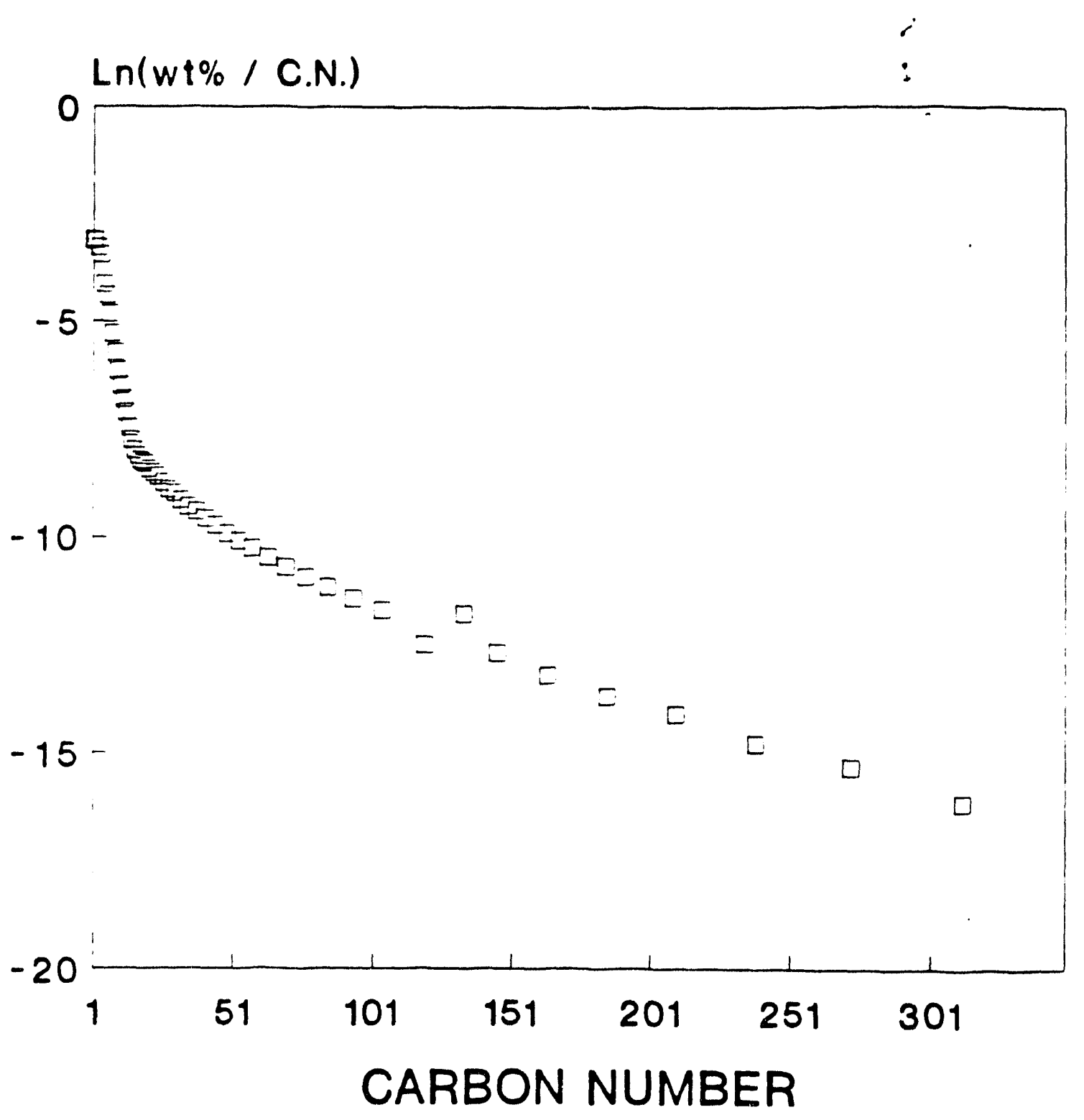

$\square$ Experimental 


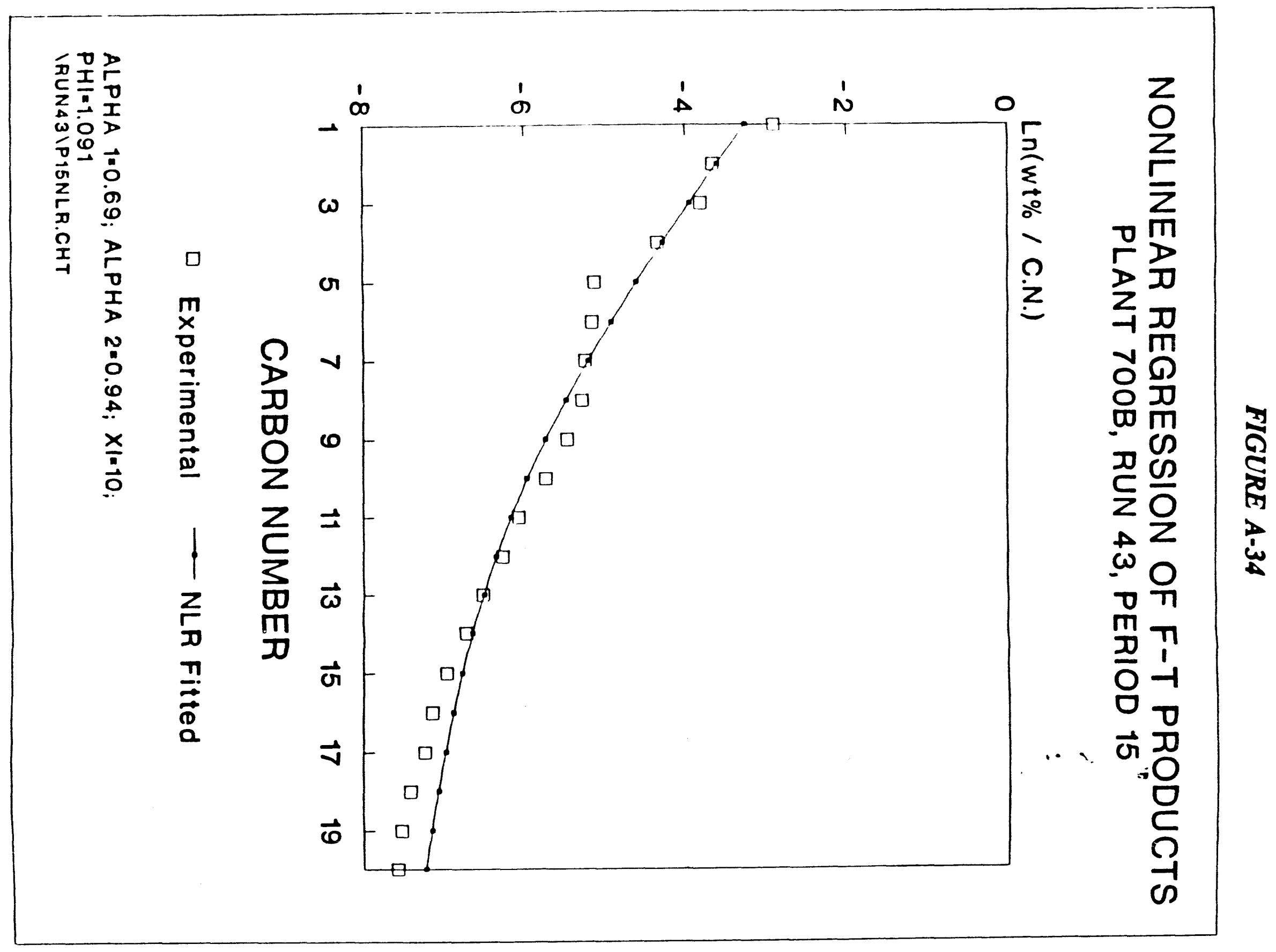




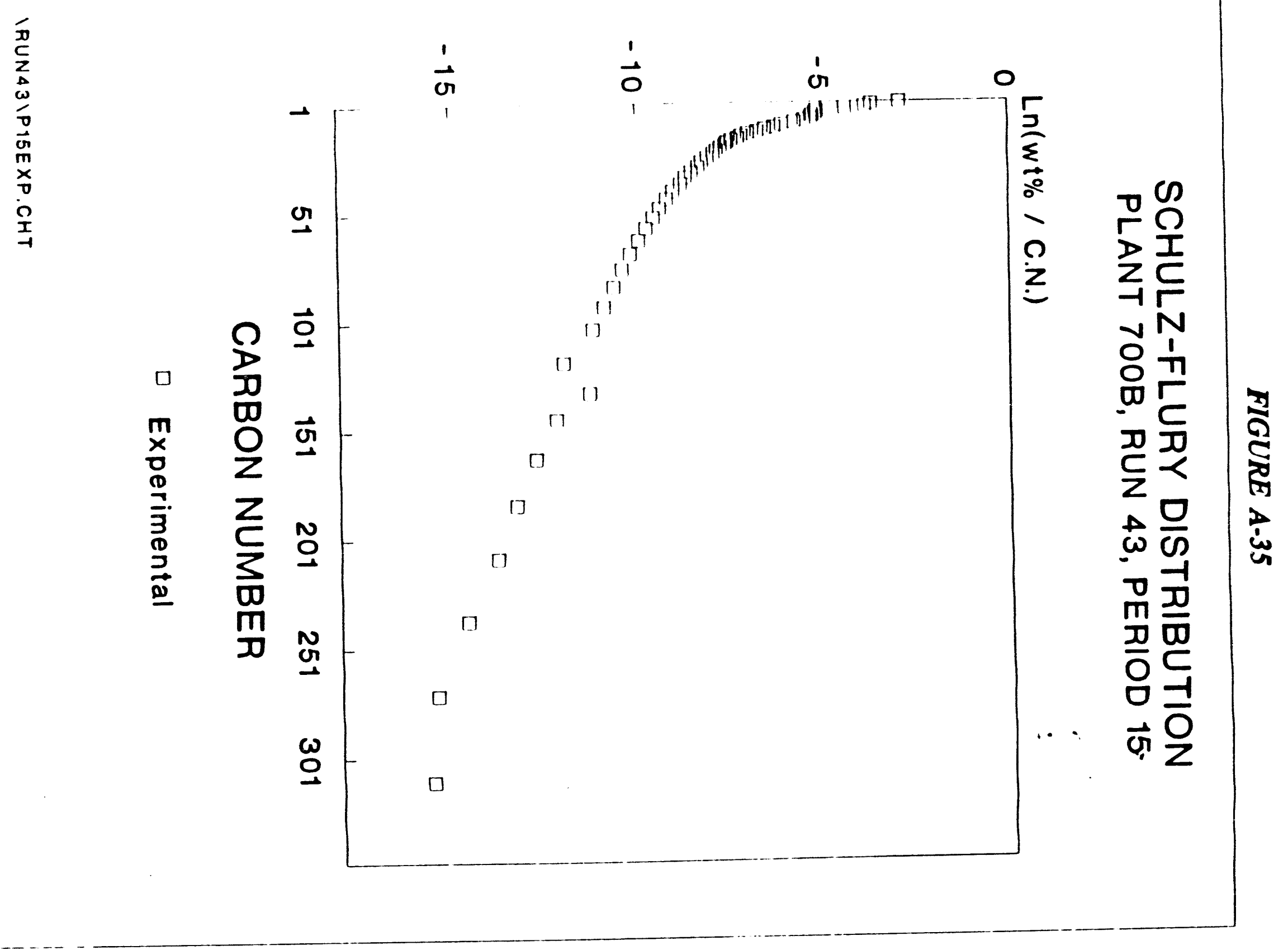


FIGURE A-36

\section{HC PRODUCT DISTRIBUTION PLANT 700B RUN 43}

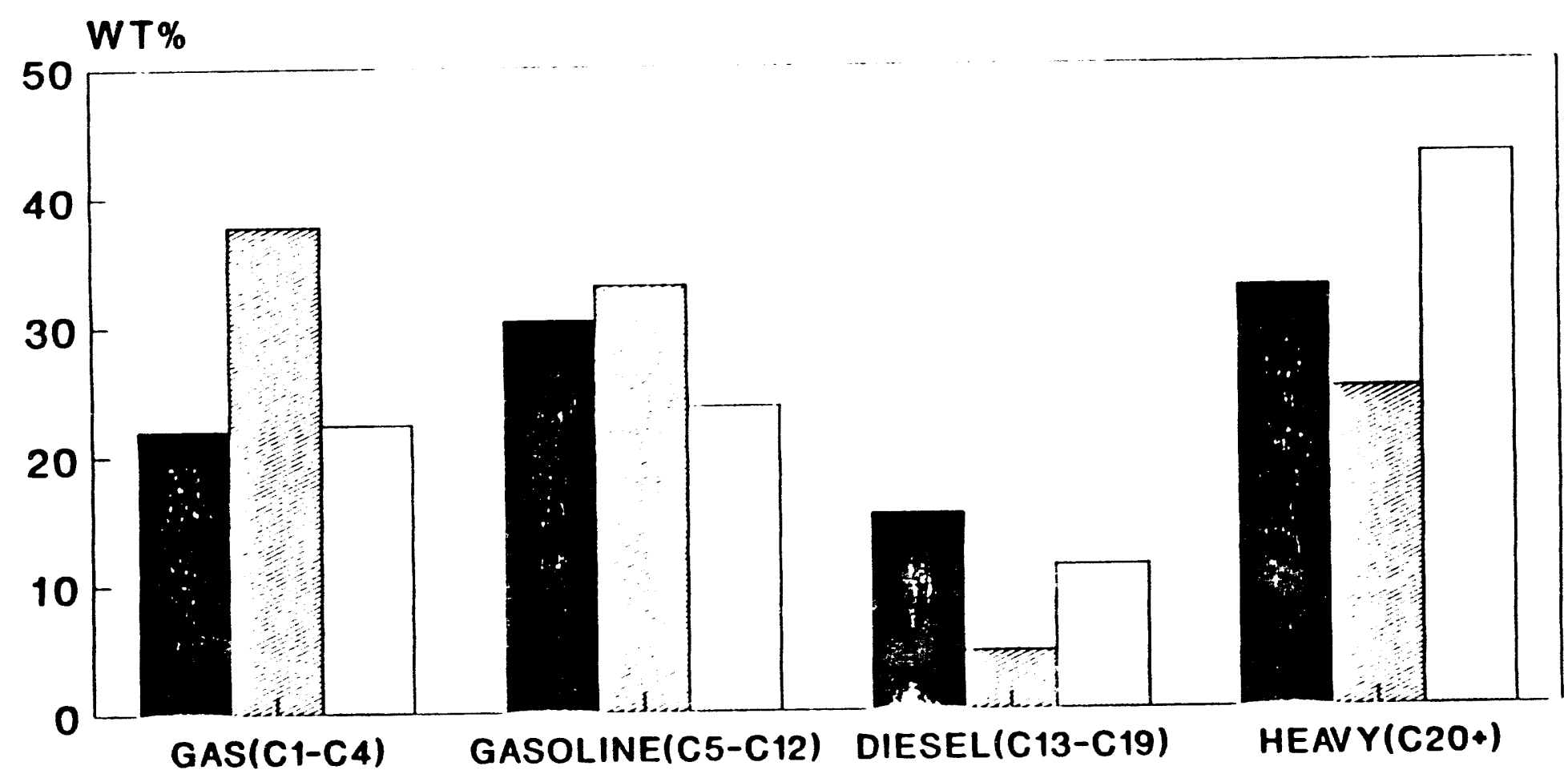

Hours On Stream 


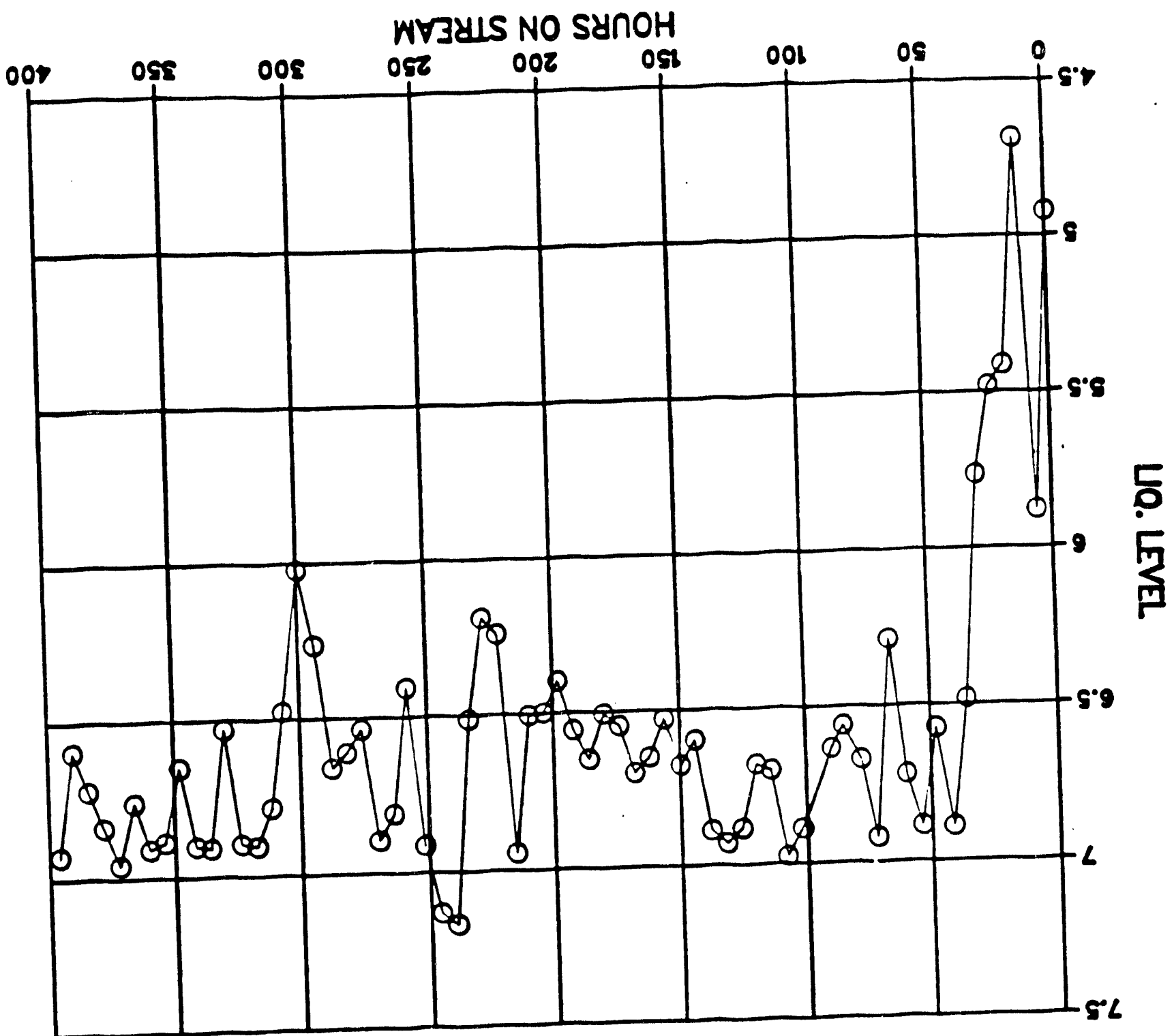

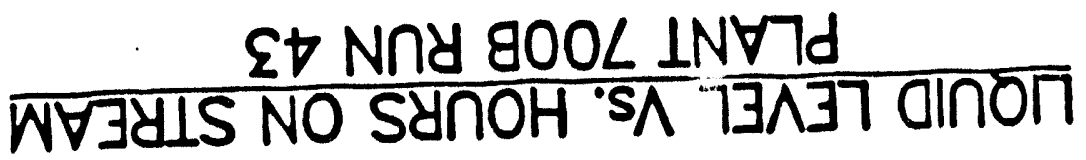


FIGURE A-38

Plant 7008, Run 43

CO Conv/Mothano 80100

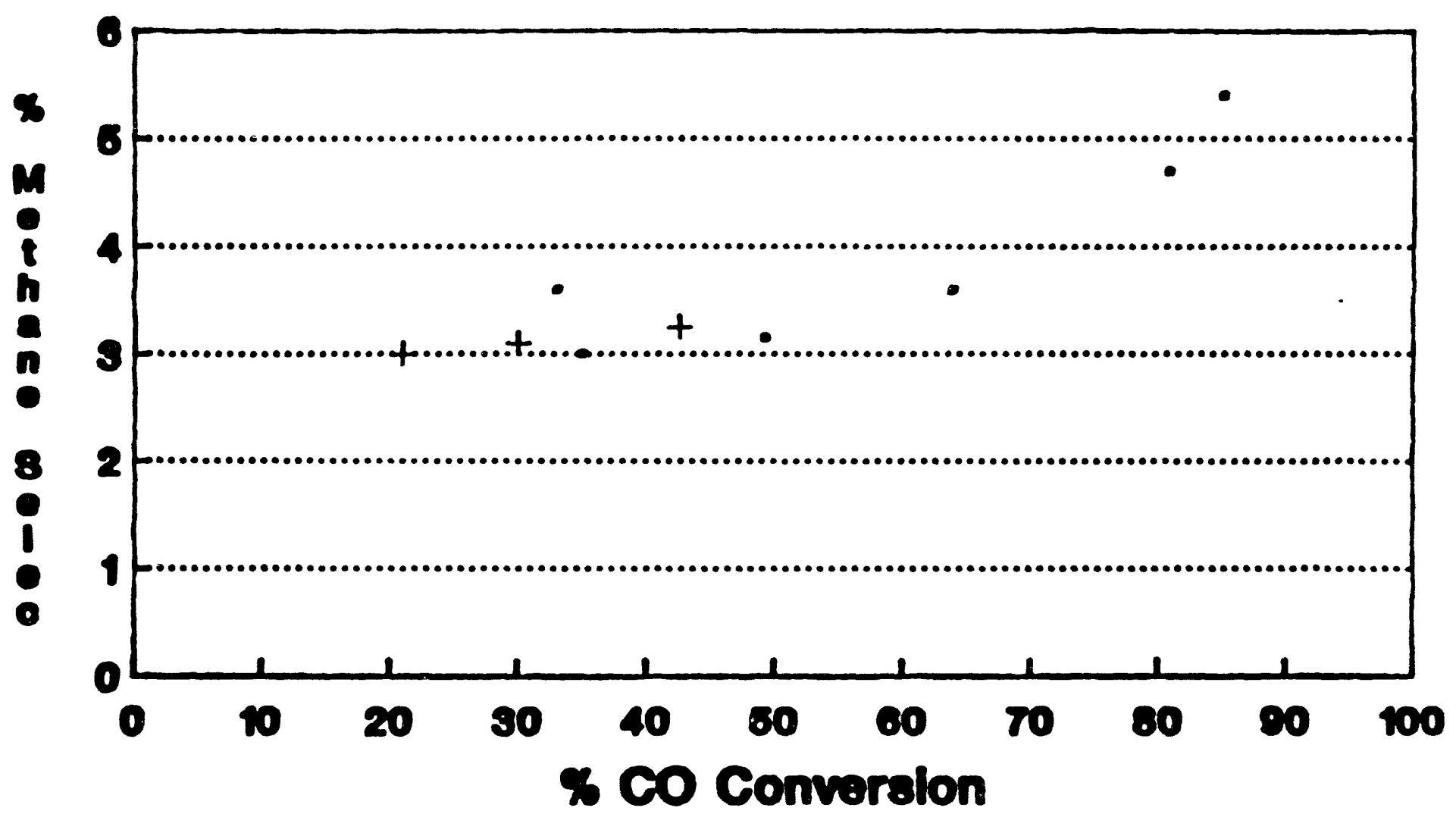

- 288 DEG C + 288 DEQ C 


\section{FIGURE A-39 \\ Plant 700B, Run 43 \\ Contact Timo/Mothane Yiold}

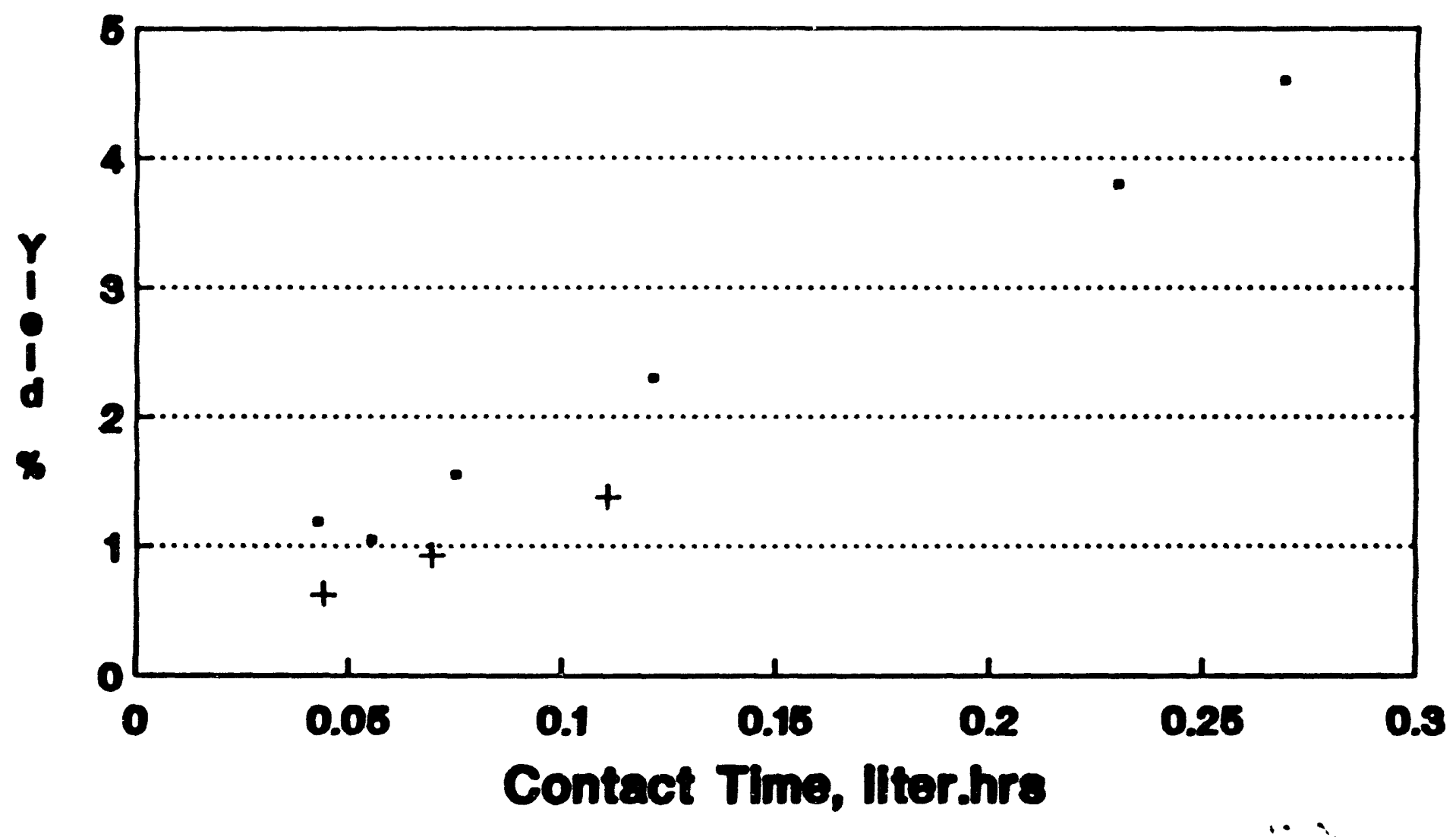

- 286 DEG C + 266 DEQ C 


\section{FIGURE A-40 \\ Plant 700B, Run 43 \\ Contact Time/Ethylono Ylold}

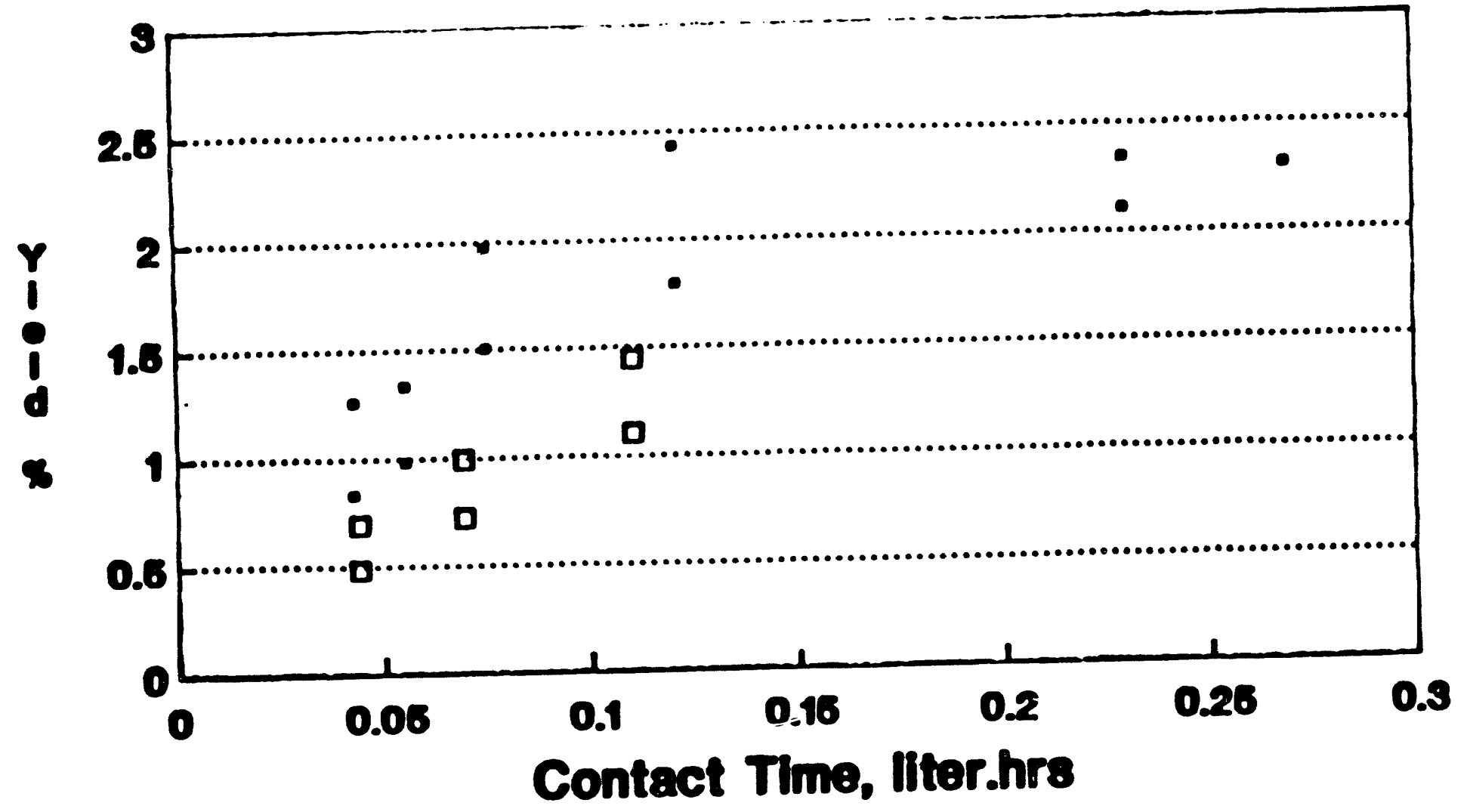

- 266 DEG C D 266 DEC C 


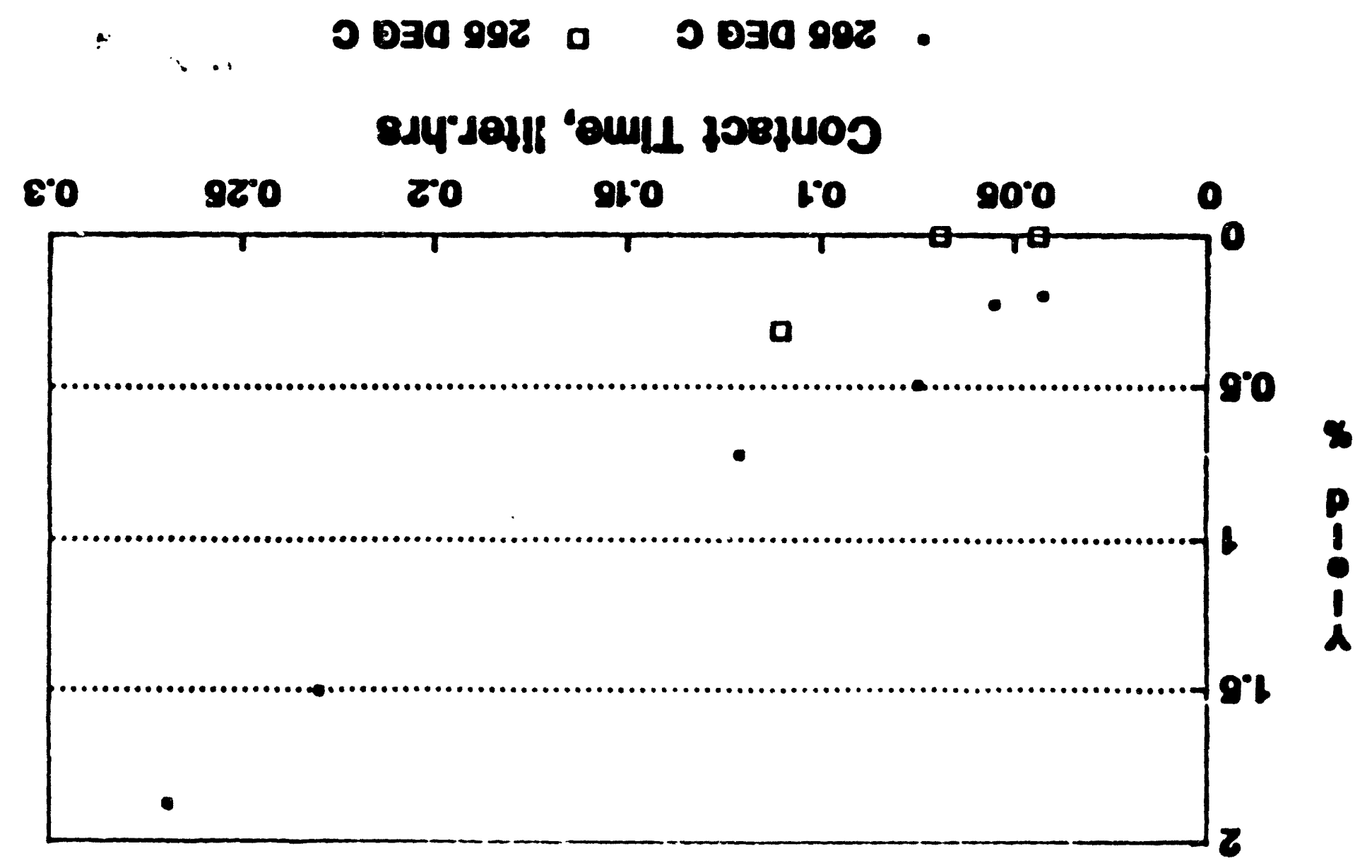

plols ousyiz/ould joojuos

\&t uny "g00L juold

It $\forall$ gd $\Lambda D J$ 


\section{FIGURE A-42 \\ Plant 700B, Run 43 \\ Contact TImo/Ethanol Ylold}

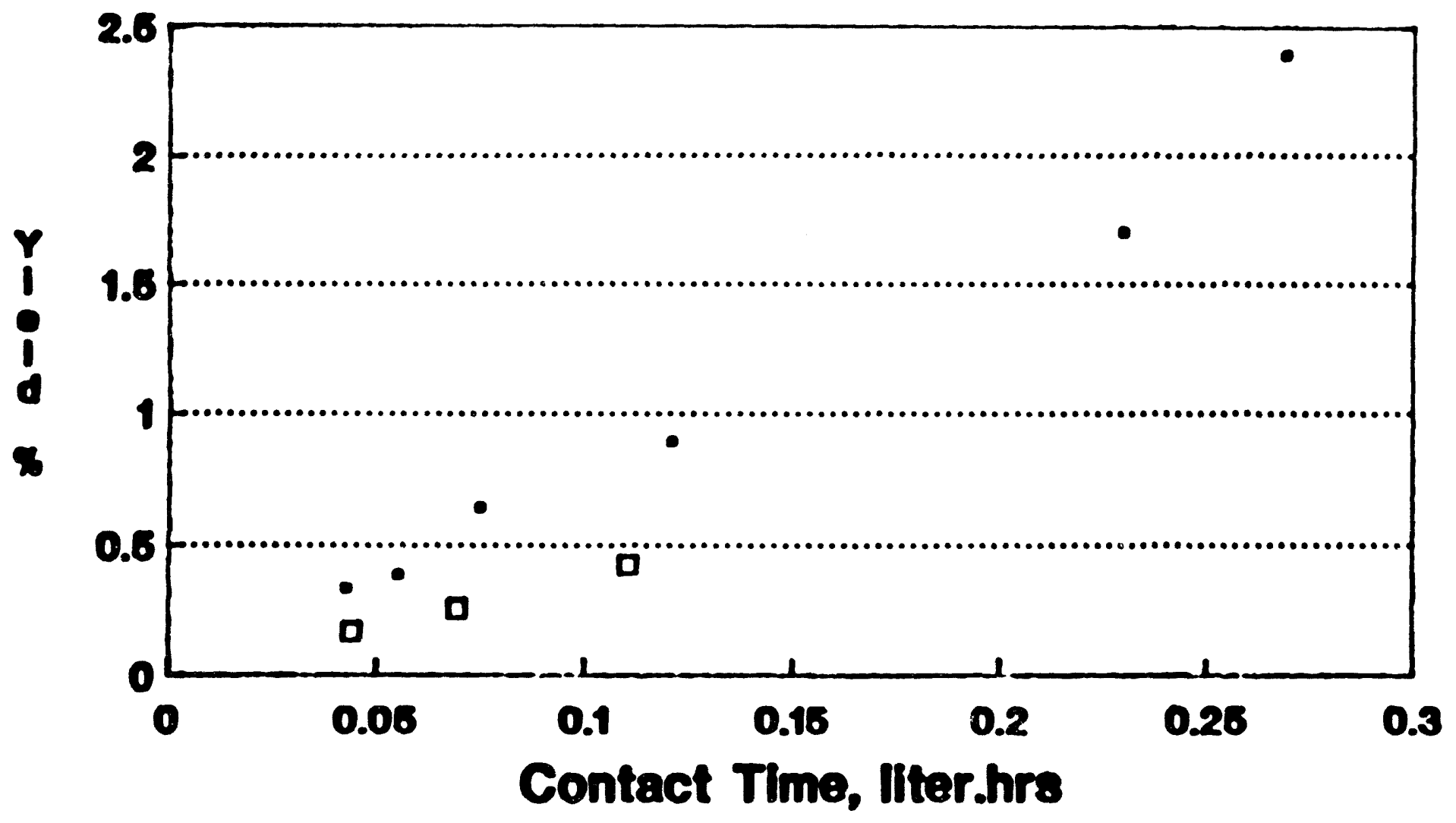

- 266 DEG C 266 DEQ C : 


\section{FIGURE A-43 \\ Plant 700B, Run 43 \\ Contact Timo/Propene Ylold}

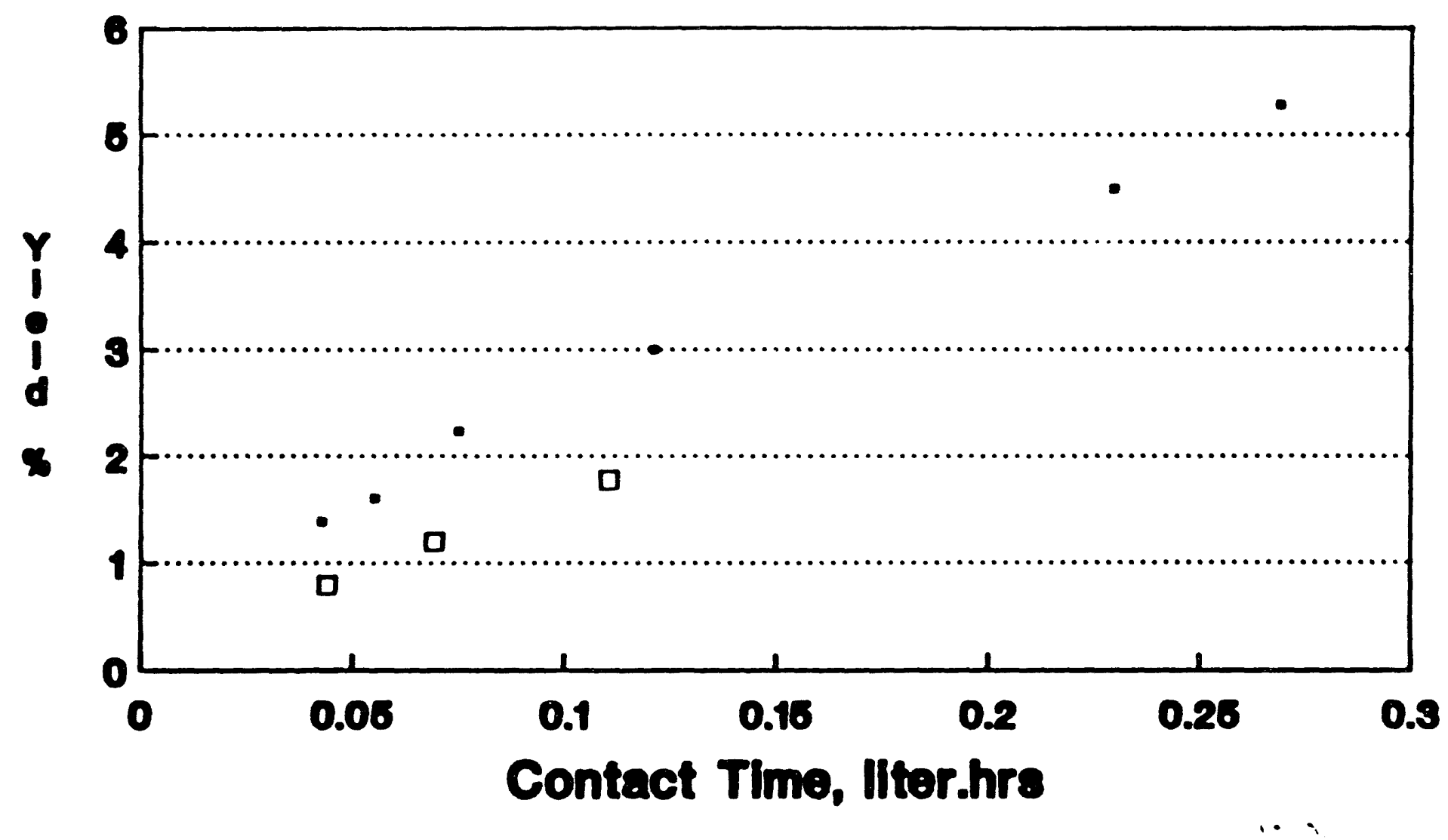

- 266 DEG C 266 DEa C 
FIGURE A-44

Plant 700B, Run 43

Contact Timo/Propane Yield

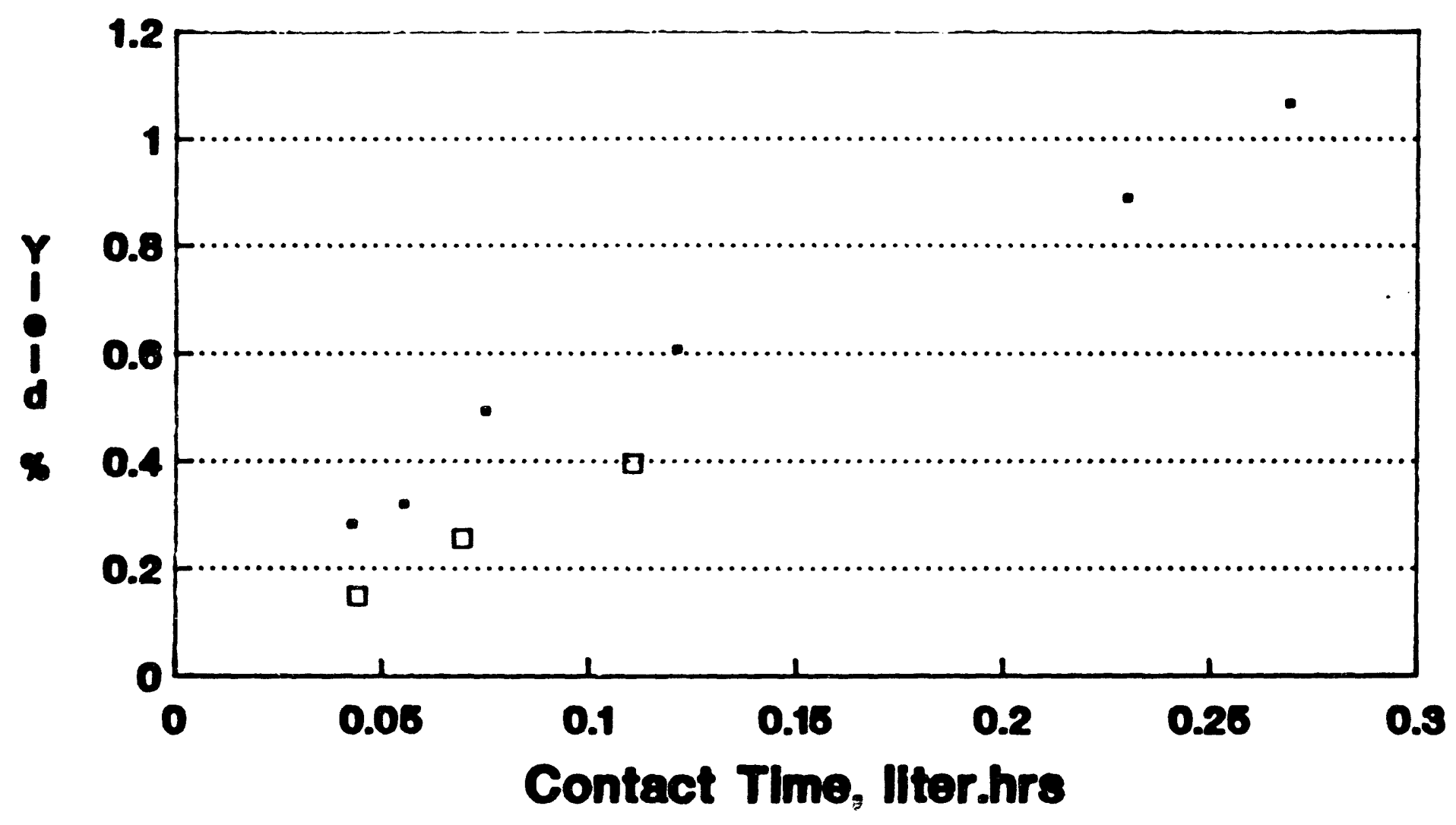

- 266 DEG C 2266 Deg C 
FIGURE A-45

Plant 700B, Run 43

Contact TImo/Propanol Ylold

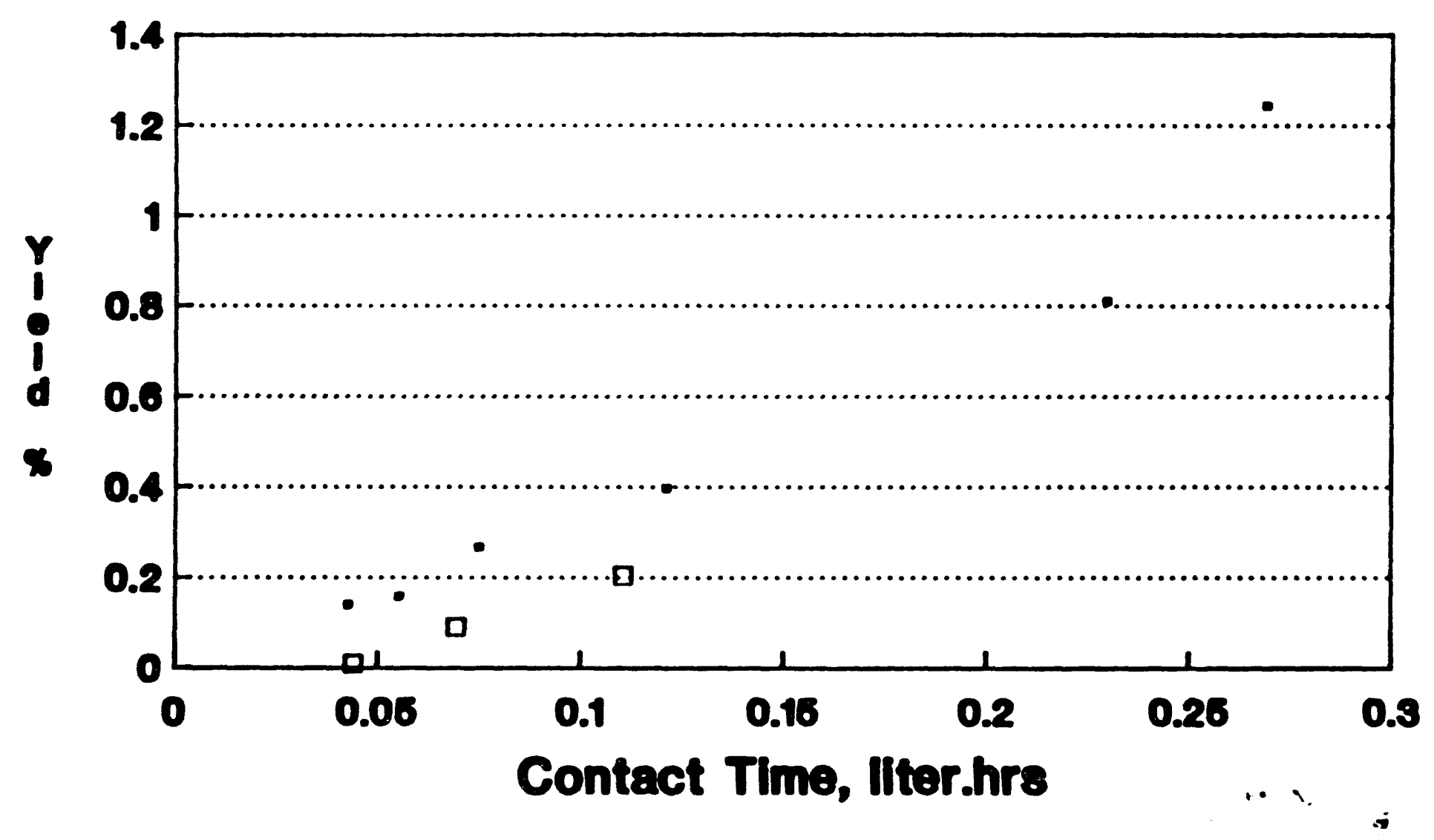

- 266 DEG C D 266 DEQ C 


\section{FIGURE A-46}

\section{Plant 700B, Run 43}

Contect Time/Butone Ylold

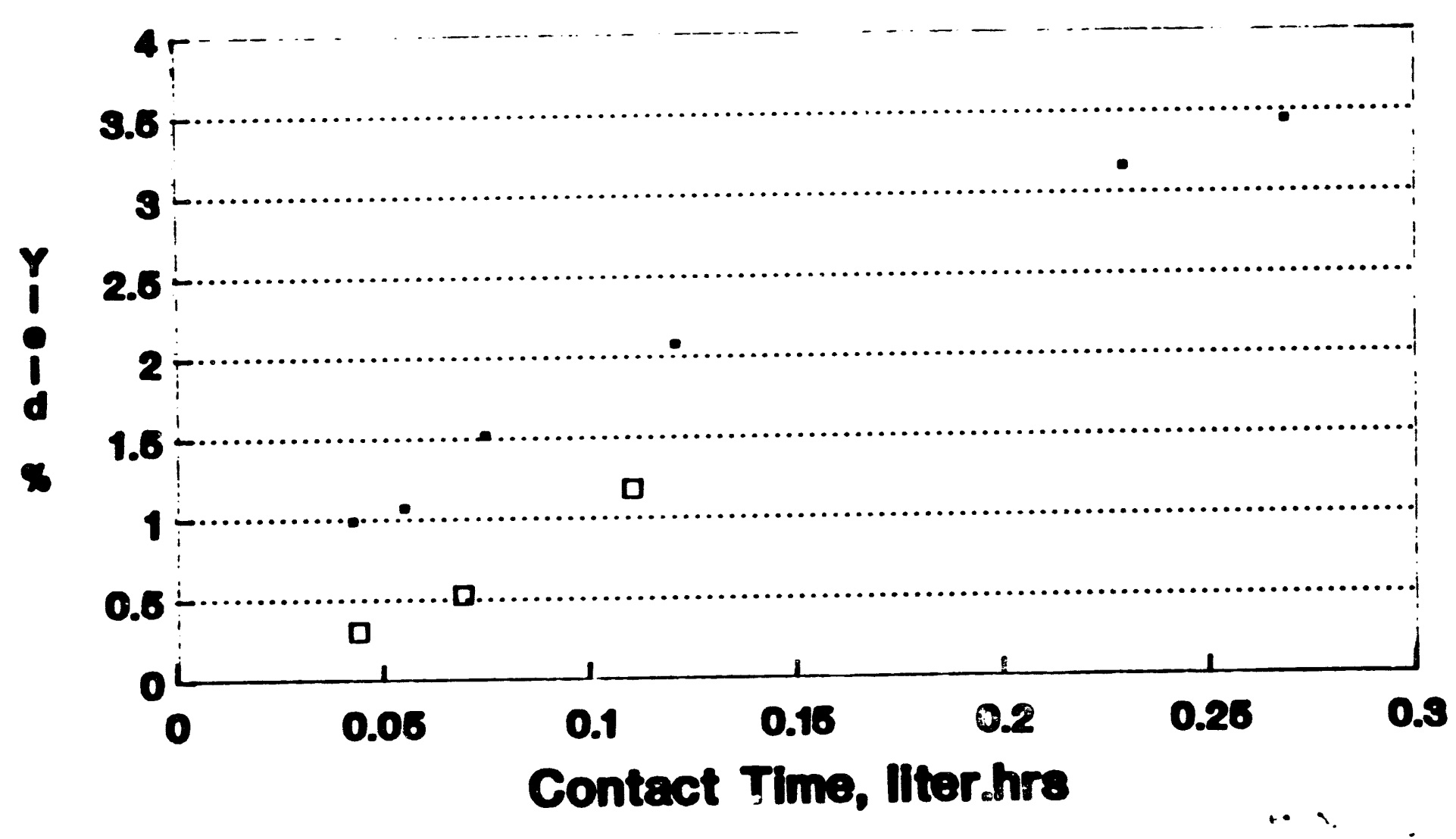

- 266 DEG C D 266 DEC C 


\section{FIGURE A-47 \\ Plant 700B, Run 43 \\ Contact TIme/Butano Yiold}

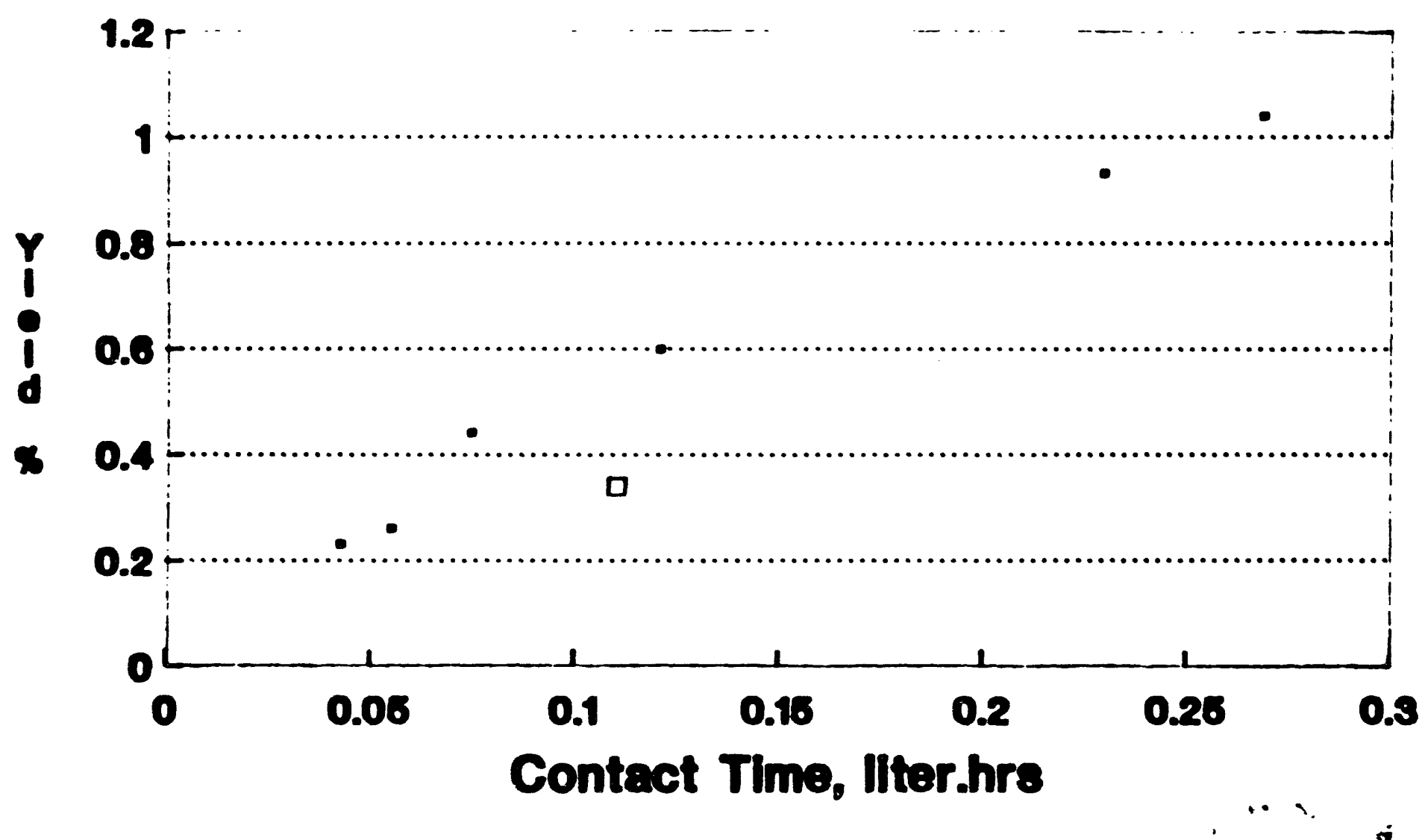

- 286 DEG C $\quad 268$ DEG C 


\section{FIGURE A-48 \\ Plant 700B, Run 43 \\ Contact TImo/Butanol Yiold}

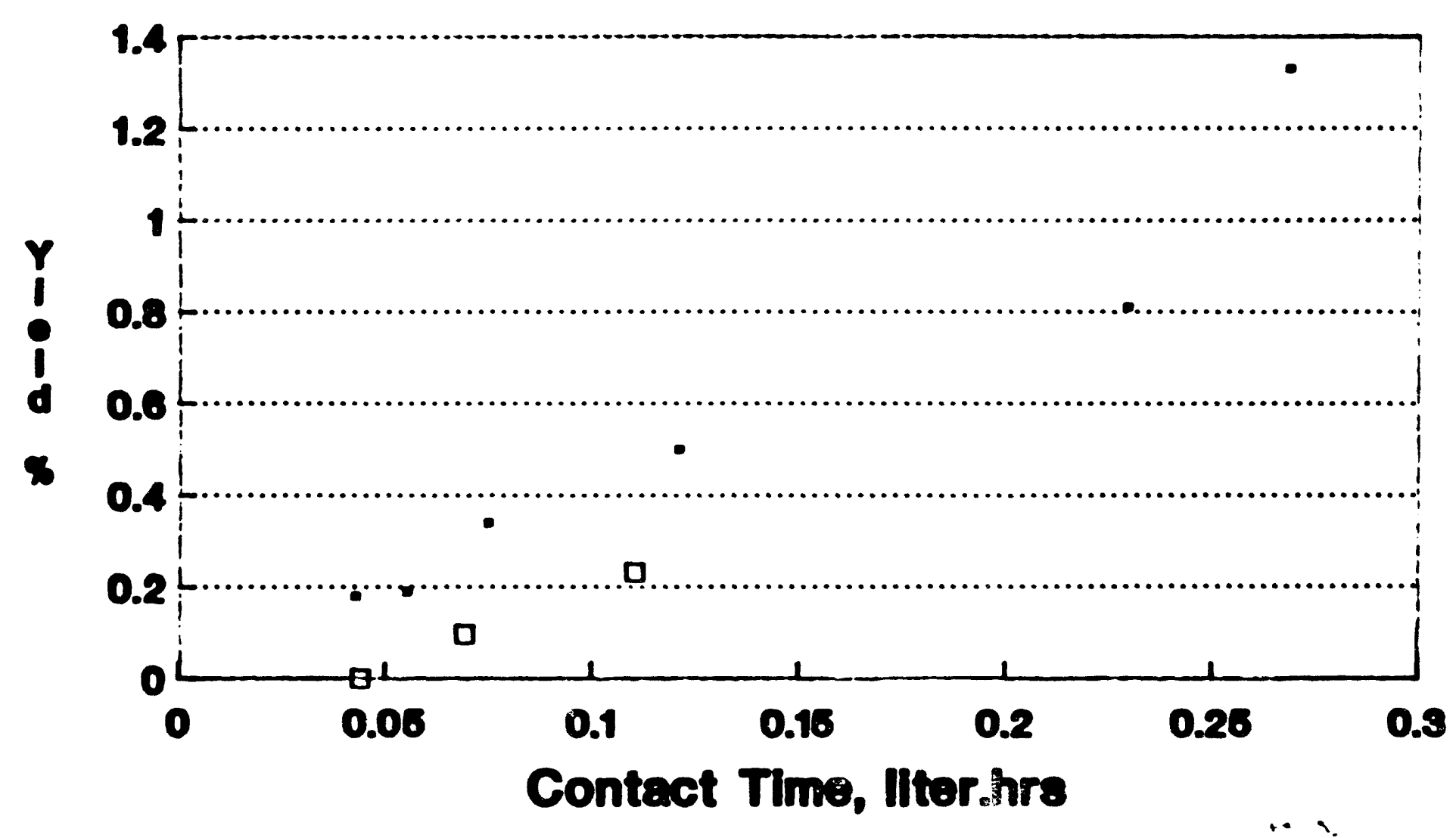

- 286 DEG C $\square 268$ DEG C 

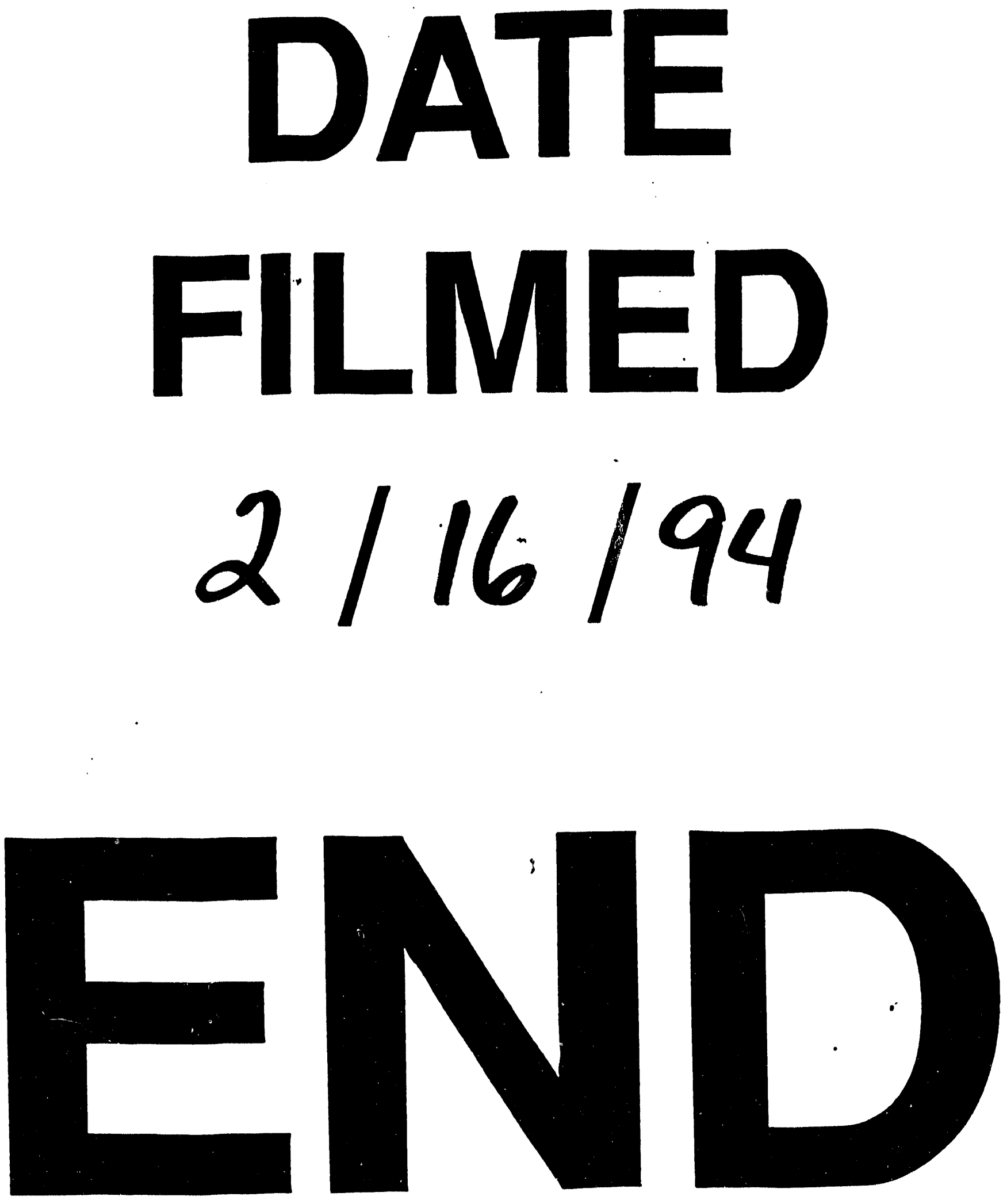
